K-teoria de operadores pseudodiferenciais na reta com símbolos semiperiódicos

Cintia Cristina da Silva

TESE APRESENTADA

AO

INSTITUTO DE MATEMÁTICA E ESTATÍSTICA

DA

UNIVERSIDADE DE SÃO PAULO

PARA

OBTENÇÃO DO GRAU DE DOUTORA

EM

MATEMÁTICA

Área de Concentração: Análise

Orientador: Prof. Dr. Severino Toscano do Rego Melo

-São Paulo, março de 2005-

Durante a elaboração deste trabalho a autora recebeu auxitio financeiro da CAPES. 



\title{
K-teoria de operadores pseudodiferenciais na reta com símbolos semiperiódicos
}

\author{
Este exemplar corresponde à redação \\ final da tese devidamente corrigida \\ e defendida por Cintia Cristina da Silva \\ e aprovada pela comissão julgadora.
}

Banca examinadora:

- Prof. Dr. Severino Toscano do Rego Melo (orientador) - IME-USP

- Profa. Dra. Cristina Cerri - IME-USP

- Prof. Dr. Ruy Exel Filho - UFSC

- Prof. Dr. Antonio Roberto da Silva - UFRJ

- Profa. Dra. Beatriz Abadie - URU

São Paulo, março de 2005. 

A minha mãe e irmãs,

Kátia e Andréa

com muito carinho. 



\section{Muitos vão orar}

E querem encontrar alguém

Que garanta ao máximo

A paz nos corações

Não há o que temer

Embora o medo exista em nós

Mas a fé nos faz mover

Montanhas com poder

Pode um milagre enfim

acontecer

Quando você acreditar

A esperança em ti

Ninguém jamais

Irá matar, depende só

Se tu quiseres crer

O medo acabou

Pois ele agia sempre em vão

A esperança em ti fará aos ares

flutuar

Não posso explicar

O que o coração de alguém

Sente quando encontra a paz

$\mathrm{E}$ a fé pra ir além

A coragem te fará vencer

Conquistando o reino em redor

É só assim que enxergarás

Que nada enfim lhe faltará

Ouça a voz a voz que diz

Contigo eu estarei

Letra da música: Se tu quiseres crer (When you believe-Prince of the Egypt) Stephen Schwartz/Kenneth Edmonds; versão: Ney Gomes 



\section{Agradecimentos}

Primeiramente a Deus por todas as bençãos recebidas durante esses anos de doutorado e por saber que sempre o terei ao meu lado.

Ao meu orientador Prof. Dr. Severino Toscano do Rego Melo pela confiança em mim depositada, pela sugestão do tema e orientação.

A minha mãe Lourdes, irmãs Kátia e Andréa e também a Tabatha e Ted pelo amor incondicional e todo apóio nos momentos difíceis.

A todos os professores que contribuíram, diretamente ou indiretamente, para minha formação. Em especial quero agradecer à Profa. Dra. Zara Issa Abud, à Profa. Dra. Roseli Fernandez e ao Prof. Dr. Daniel Tausk.

Ao Prof. Dr. Ruy Exel por todas as dicas e explicações durante a elaboração deste trabalho.

Ao David, Tadeu, Fábio, Bianconi, Toscano e especialmente à Cristina e à Martha pela ajuda nos seminários de $C^{*}$-álgebras e Álgebra de Operadores.

A Olga, ao Rudimar e a Vera pelo companheirismo dentro e fora da faculdade.

A todos os amigos do IME, em especial à Andréia, ao Zeca, à Irina, ao Santos, à Neusa e ao Seu Angelo. 



\section{Resumo}

Seja $\mathcal{A}$ a menor $C^{*}$-álgebra de $\mathcal{L}\left(L^{2}(\mathbb{R})\right)$, onde $\mathcal{L}\left(L^{2}(\mathbb{R})\right)$ denota a álgebra dos operadores limitados em $L^{2}(\mathbb{R})$, contendo: (i) todas multiplicações $a(M), a \in C([-\infty,+\infty])$, (ii) todas multiplicações $e^{i j M}, j \in \mathbb{Z}$, e (iii) todos os operadores da forma $F^{-1} b(M) F$, onde $F$ denota a transformada de Fourier e $b \in C([-\infty,+\infty])$. É conhecido que o símbolo principal se estende a um $*$-homomorfismo sobrejetivo $\sigma: \mathcal{A} \rightarrow C\left(M_{A}\right)$, onde $M_{A}$ é uma certa compactificação de $\mathbb{R} \times\{-1,1\}$. É também conhecido que $\mathcal{E}=\operatorname{Ker} \sigma$ contém o ideal dos operadores compactos $\mathcal{K}$ e que o quociente $\mathcal{E} / \mathcal{K}$ é isomorfo a $C\left(S^{1} \times\{-1,1\}\right) \otimes \mathcal{K}$. Usando a forma explícita desses dois isomorfismos, fomos capazes de calcular os "connecting mappings" da sequência exata cíclica de seis termos de K-teoria associada ao homomorfismo $\sigma$ e então provamos que $K_{0}(\mathcal{A}) \cong \mathbb{Z}$ e $K_{1}(\mathcal{A}) \cong \mathbb{Z} \oplus \mathbb{Z}$.

O isomorfismo $\mathcal{E} / \mathcal{K} \cong C\left(S^{1} \times\{-1,1\}\right) \otimes \mathcal{K}$ pode ser estendido a um $*$-homomorfismo $\gamma: \mathcal{A} \rightarrow C\left(S^{1} \times\{-1,1\}\right) \otimes \mathcal{L}_{\mathbb{Z}}$, onde $\mathcal{L}_{\mathbb{Z}}$ denota a álgebra dos operadores limitados em $L^{2}(\mathbb{Z})$. Provamos que a imagem de $\gamma$ é isomorfa a soma de duas cópias do produto cruzado $C([-\infty,+\infty]) \rtimes \mathbb{Z}$ onde $\alpha$ denota um automorfismo em $C([-\infty,+\infty])$ definido por $(\alpha f)(x)=f(x+1), f \in C([-\infty,+\infty]), x \in \mathbb{R}$. Usando a sequência exata de PimsnerVoiculescu, pudemos calcular a K-teoria da imagem de $\gamma$. O que nos levou a uma segunda prova de que $K_{0}(\mathcal{A}) \cong \mathbb{Z}$ e $K_{1}(\mathcal{A}) \cong \mathbb{Z} \oplus \mathbb{Z}$. 



\section{Abstract}

Let $\mathcal{A}$ denote the smallest $C^{*}$-subalgebra of the algebra of all bounded operators on $L^{2}(\mathbb{R})$ containing: (i) all multiplications $a(M)$ by functions $a \in C([-\infty,+\infty])$, (ii) all multiplications $e^{i j M}, j \in \mathbb{Z}$, and (iii) all operators of the form $F^{-1} b(M) F$, where $F$ denotes the Fourier transform and $b \in C([-\infty,+\infty])$. It is known that the principal symbol mapping extends to a surjective $C^{*}$-homomorphism $\sigma: \mathcal{A} \rightarrow C\left(M_{A}\right)$, onde $M_{A}$ is a certain compactification of $\mathbb{R} \times\{-1,1\}$. It is also known that $\mathcal{E}=\operatorname{Ker} \sigma$ contains the compact ideal $\mathcal{K}$ and that the quotient $\mathcal{E} / \mathcal{K}$ is isomorphic to $C\left(S^{1} \times\{-1,1\}\right) \otimes \mathcal{K}$. Using the explicit form of these two isomorphisms, we are able to compute the connecting mappings in the cyclic exact sequence in K-theory associated to the homomorphism $\sigma$ and the prove that $K_{0}(\mathcal{A}) \cong \mathbb{Z}$ and $K_{1}(\mathcal{A}) \cong \mathbb{Z} \oplus \mathbb{Z}$.

The isomorphism $\mathcal{E} / \mathcal{K} \cong C\left(S^{1} \times\{-1,1\}\right) \otimes \mathcal{K}$ can be to extended to a $C^{*}$-homomorphism $\gamma: \mathcal{A} \rightarrow C\left(S^{1} \times\{-1,1\}\right) \otimes \mathcal{L}_{\mathbb{Z}}$, where $\mathcal{L}_{\mathbb{Z}}$ denotes the algebra of bounded operators on $L^{2}(\mathbb{Z})$. We prove that the image of $\gamma$ is isomorphic to the direct sum of two copies of the crossed product $C([-\infty,+\infty]) \rtimes \mathbb{Z}$ where $\alpha$ denotes the automorphism on $C([-\infty,+\infty])$ defined by $(\alpha f)(x)=f(x+1), \stackrel{\alpha}{f} \in C([-\infty,+\infty]), x \in \mathbb{R}$. Using the Pimsner-Voiculescu exact sequence, we then compute the K-theory of the image of $\gamma$. That leads to a second proof that $K_{0}(\mathcal{A}) \cong \mathbb{Z}$ and $K_{1}(\mathcal{A}) \cong \mathbb{Z} \oplus \mathbb{Z}$. 


\section{Sumário}

Notações

Introdução

$\begin{array}{llr}1 & \text { A álgebra } \mathcal{A} & 17\end{array}$

1.1 Ideal comutador . . . . . . . . . . . . . . . . . . . 18

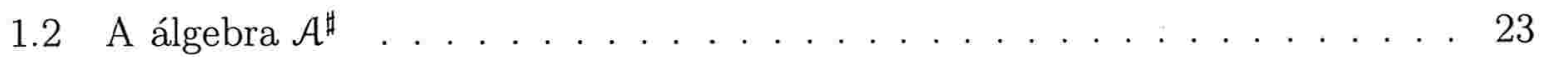

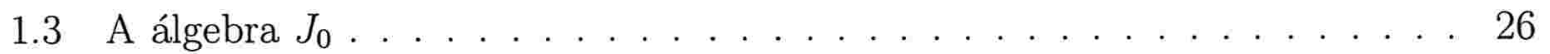

1.4 O espaço símbolo de $\mathcal{A} \ldots \ldots \ldots \ldots$. . . . . . . . . . . . . . 31

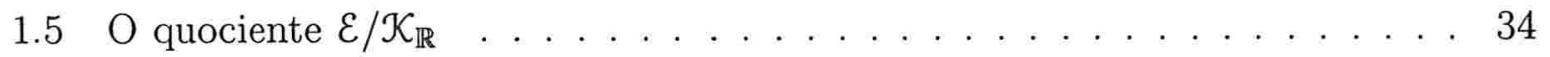

2 A álgebra ${ }_{2} \quad 40$

2.1 O espaço símbolo de $\mathrm{C} \ldots \ldots \ldots \ldots \ldots$

2.2 Espaços de Hardy e operadores de Toeplitz . . . . . . . . . . . . . . . . . 42

2.3 Índice de Fredholm e a K-teoria de e . . . . . . . . . . . . . . . . 45

3 K-teoria de $\mathcal{A} \quad 52$

$3.1 \quad$ A K-teoria de $\mathcal{A} / \mathcal{E}$ e $\mathcal{E} / \mathcal{K}_{\mathbb{R}} \ldots \ldots \ldots \ldots \ldots$

3.2 A K-teoria de $\mathcal{A} / \mathcal{K}_{\mathbb{R}}$ e $\mathcal{A} \ldots \ldots \ldots \ldots \ldots$ 



\section{Notações}

Durante este trabalho adotamos a convenção de que um ideal é sempre um ideal bilateral.

- $\mathcal{L}(E):=\{$ operadores limitados sobre o espaço de Banach $E\}$.

- $\mathcal{K}(E):=\{$ operadores compactos sobre o espaço de Banach $E\}$.

- $\mathcal{K}_{E}:=\mathcal{K}\left(L^{2}(E)\right)$.

- $\mathcal{L}_{E}:=\mathcal{L}\left(L^{2}(E)\right)$.

- $S^{1}:=$ círculo unitário em $\mathbb{C}$.

- $S_{+}^{1}=S^{1} \times\{+\infty\}$.

- $S_{-}^{1}=S^{1} \times\{-\infty\}$.

- $C(X):=\{$ funções complexas contínuas sobre $X\}$.

- $C^{\infty}(X):=\{$ funções complexas infinitamente diferenciáveis sobre $X\}$.

- $C_{c}^{\infty}(X):=\left\{\right.$ funções de $C^{\infty}(X)$ com suporte compacto $\}$.

- $C S(\mathbb{R}):=\{$ funções contínuas de $\mathbb{R}$ em $\mathbb{C}$ com limites em $+\infty$ e $-\infty\} \cong C([-\infty,+\infty])$, $[-\infty,+\infty]$ denotando a compactificação de $\mathbb{R}$ que se obtém acrescentando os pontos $-\infty$ e $+\infty$.

- $C S(\mathbb{Z}):=\{$ sequências complexas indexadas por $\mathbb{Z}$ com limites em $+\infty$ e $-\infty\}$.

- $P_{2 \pi}:=\{$ funções contínuas de $\mathbb{R}$ em $\mathbb{C} 2 \pi$-periódicas $\}$. 

- $C_{0}(\mathbb{R}):=\{$ funções contínuas de $\mathbb{R}$ em $\mathbb{C}$ com limite zero em $\pm \infty\}$.

- $\mathcal{S}(\mathbb{R}):=\left\{\phi \in C^{\infty}(\mathbb{R}): \sup _{x}\left|x^{\alpha} \phi^{\beta}(x)\right|<\infty, \alpha, \beta \in \mathbb{N}\right\}$.

- $F$ denota a Transformada de Fourier em $\mathbb{R}$ :

$$
F u(x)=\frac{1}{\sqrt{2 \pi}} \int_{\mathbb{R}} e^{-i x t} u(t) d t .
$$

- Id denota o operador identidade em um espaço de Hilbert.

- ind denota o índice de um operador de Fredholm.

- $w$ denota o número de rotação de uma curva fechada em $\mathbb{C} \backslash\{0\}$.

- $\delta_{0}$ é a aplicação exponencial da sequência exata cíclica de seis termos de K-teoria.

- $\delta_{1}$ é a aplicação do índice da sequência exata cíclica de seis termos de K-teoria.

- * denota a operação convolução.

- $T^{*}$ denota o operador adjunto de $T$.

- $\hat{T}=F T$.

- $<\cdot, \cdot>$ denota produto interno.

- $a(M)$ denota o operador de multiplicação por $a$. Neste trabalho utilizamos $a(M) \in \mathcal{L}_{\mathbb{R}}$, isto é $a(M) u(x)=a(x) u(x), a \in L^{\infty}(\mathbb{R})$.

$a(M) \in \mathcal{L}_{\mathbb{Z}}$, isto é $a(M)\left(u_{j}\right)_{j}=\left(a(j) u_{j}\right)_{j}, a: \mathbb{Z} \rightarrow \mathbb{C}$ limitada. $a(M) \in \mathcal{L}_{S^{1}}$, isto é $a(M) u(x)=a(x) u(x), a \in L^{\infty}\left(S^{1}\right)$.

- $A u t(A)$ denota o conjunto dos automorfismos de $A, A$ uma $C^{*}$ - álgebra.

- $G L(A)$ denota o grupo dos elementos inversíveis de $A, A$ uma $C^{*}$ - álgebra com unidade.

- $U(A)$ denota o grupo dos elementos unitários de $A, A$ uma $C^{*}$ - álgebra com unidade.

- $\tilde{A}$ denota a unitização da $C^{*}$ - álgebra $A$.

- $\bar{\varphi}$ : dado o $*$-homomorfismo $\varphi: A \rightarrow B$ temos $\bar{\varphi}: \tilde{A} \rightarrow \tilde{B}$ onde $\bar{\varphi}\left(a+\alpha 1_{\tilde{A}}\right)=$ $\varphi(a)+\alpha 1_{\tilde{B}}, a \in A$ e $\alpha \in \mathbb{C}$. 


\section{Introdução}

Dado o espaço de Hilbert $L^{2}(\mathbb{R})$, seja $\mathcal{L}\left(L^{2}(\mathbb{R})\right)$ o conjunto dos operadores limitados de $L^{2}(\mathbb{R})$. É conhecido que $\mathcal{L}\left(L^{2}(\mathbb{R})\right)$ é uma $C^{*}$ - álgebra (veja, por exemplo, [28], exemplo 2.1.3).

Denotemos por $\mathcal{A}$ a $C^{*}$ - subálgebra de $\mathcal{L}\left(L^{2}(\mathbb{R})\right)$ gerada pelas seguintes classes de operadores:

1. As multiplicações $b(M)$ por funções $b \in C S(\mathbb{R})$.

2. As multiplicações $e^{i j M}, j \in \mathbb{Z}$.

3. Os operadores da forma $b(D):=F^{-1} b(M) F, F$ denotando a transformada de Fourier e $b$ como no item anterior.

Denotemos por $S L$ a $C^{*}$ - subálgebra de $\mathcal{L}\left(L^{2}\left(S^{1}\right)\right)$ gerada pelos operadores a $(M)$ de multiplicação por funções $a \in C^{\infty}\left(S^{1}\right)$ e por todos os operadores da forma $b\left(D_{\theta}\right):=$ $F_{d}^{-1} b(M) F_{d}$, onde $F_{d}: L^{2}\left(S^{1}\right) \longrightarrow L^{2}(\mathbb{Z})$ denota a transformada de Fourier discreta e $b(M) \in \mathcal{L}\left(L^{2}(\mathbb{Z})\right)$ o operador de multiplicação por uma sequência $\left(b_{j}\right)_{j \in \mathbb{Z}}$ que possua limites quando $j$ tende a $+\infty \mathrm{e}-\infty$. É sabido que $S L$ contém o ideal $\mathcal{K}_{S^{1}}$ dos operadores compactos de $L^{2}\left(S^{1}\right)$ e que o quociente $S L / \mathcal{K}_{S^{1}}$ é isomorfo a álgebra das funções contínuas em $S^{1} \times\{-1,1\}$, com isomorfismo determinado por

$$
a(M) \longmapsto((z, \pm 1) \mapsto a(z)) \quad \text { e } \quad b(D) \longmapsto\left((z, \pm 1) \mapsto \lim _{j \rightarrow \pm \infty} b_{j}\right) .
$$

Seja $\mathcal{E}$ o menor ideal fechado contendo todos os comutadores de $\mathcal{A}$. Então $\mathcal{E}$ contém o ideal $\mathcal{K}_{\mathbb{R}}$ dos operadores compactos em $L^{2}(\mathbb{R})$ e é isomorfo [13] ao produto tensorial de $C^{*}$ - álgebras $S L \otimes \mathcal{K}_{\mathbb{Z}}$, onde $\mathcal{K}_{\mathbb{Z}}$ é o ideal dos operadores compactos em $L^{2}(\mathbb{Z})$ (a construção explícita de um isomorfismo depende da escolha de um isomorfismo entre 
$L^{2}(\mathbb{R})$ e $\left.L^{2}\left(S^{1}\right) \otimes L^{2}(\mathbb{Z})\right)$. Combinando-se esse isomorfismo com o do parágrafo anterior e usando que $\mathcal{K}_{\mathbb{R}}$ é isomorfo a $\mathcal{K}_{S^{1}} \otimes \mathcal{K}_{\mathbb{Z}}$, obtém-se:

$$
\mathcal{E} / \mathcal{K}_{\mathbb{R}} \cong C\left(S^{1} \times\{-1,1\}\right) \otimes \mathcal{K}_{\mathbb{Z}}
$$

Decorre de resultados gerais sobre "álgebras de comparação" , que são certas $C^{*}$ álgebras geradas por operadores pseudodiferenciais em variedades, estudadas por Cordes e colaboradores [9], que $\mathcal{K}_{\mathbb{R}} \subset \mathcal{E}$ e que existe um isomorfismo

$$
\mathcal{A} / \mathcal{E} \cong C(X \times\{-\infty,+\infty\}),
$$

(onde $X=\left\{\left(x, e^{i x}\right): x \in \mathbb{R}\right\} \cup\left(\{-\infty,+\infty\} \times S^{1}\right)$ é um subconjunto fechado de $\left.[-\infty,+\infty] \times S^{1}\right)$ determinado por:

1. $\left.[a(M)]_{\mathcal{E}} \mapsto\left(\left(x, e^{i y}\right), \pm \infty\right) \mapsto a(y), \forall\left(x, e^{i y}\right) \in X\right)$, se $a$ é $2 \pi$-periódica.

2. $\left.[b(M)]_{\mathcal{E}} \mapsto\left(\left(x, e^{i y}\right), \pm \infty\right) \mapsto b(x), \forall\left(x, e^{i y}\right) \in X\right)$, se $b \in C S(\mathbb{R})$.

3. $[b(D)]_{\varepsilon} \mapsto((m, \pm \infty) \mapsto b( \pm \infty), \forall m \in X)$, se $b \in C S(\mathbb{R})$.

Denotamos, acima, por $[A]_{\mathcal{E}}$ a classe de $A \in \mathcal{A}$ no quociente $\mathcal{A} / \mathcal{E}$.

O resultado central deste trabalho é o cálculo da K-teoria de $\mathcal{A}$, e isto é feito nos Capítulos 3 e 4 . Nos Capítulos 1 e 2 demonstramos, ou citamos sem demonstrar, os resultados preliminares necessários para esse trabalho. A seguir detalhamos o conteúdo de cada capítulo.

No Capítulo 1, provamos que

$$
\mathcal{K}_{\mathbb{R}} \subset \mathcal{E} \subset \mathcal{A}
$$

e estabelecemos o isomorfismo (2). O que difere esse capítulo do trabalho de [13] é que para demonstrarmos que $\mathcal{K}_{\mathbb{R}} \subset \mathcal{E}$ e a existência do isomorfismo (2) não invocamos resultados sobre álgebras de comparação, embora utilizemos muitas das idéias e técnicas de $[9,10]$. Para descrevermos o isomorfismo (1) utilizaremos o Teorema 2.6 de [13], que será enunciado mas não demonstrado.

Denotemos por $\mathcal{C}$ a $C^{*}$ - subálgebra de $\mathcal{L}\left(L^{2}(\mathbb{R})\right)$ gerada pelas seguintes classes de operadores:

1. As multiplicações $a(M)$ por funções $a \in C S(\mathbb{R})$. 
2: Os operadores da forma $b(D):=F^{-1} b(M) F, F$ denotando a transformada de Fourier e $b$ como no item anterior.

No Capítulo 2 provamos que $\mathcal{K}_{\mathbb{R}} \subset \mathcal{C}$ e estabelecemos o isomorfismo

$$
\mathcal{C} / \mathcal{K}_{\mathbb{R}} \cong C\left(M_{C}\right)
$$

(onde $\left.M_{C}=\{(x, y) \in[-\infty,+\infty] \times[-\infty,+\infty]:|x|+|y|=\infty\}\right)$. Introduzimos, também, nesse capítulo um pouco sobre a teoria de espaços de Hardy (na reta e no círculo). Essa teoria foi utilizada na demonstração de que, se $T \in \mathcal{C}$ é um operador de Fredholm, temos $\operatorname{ind}(T)=w\left(\sigma_{T}\right)$ onde ind é o índice de Fredholm de $T, w$ é o número de rotação e $\sigma$ é a composição do isomorfismo de Gelfand com a projeção canônica (observamos que, como $M_{C}$ é homeomorfo ao círculo, faz sentido calcular $w\left(\sigma_{T}\right)$ ). Utilizaremos os resultados obtidos nesse capítulo nos capítulos que seguem.

A álgebra $\mathcal{C}$ foi estudada por Cordes e Herman [12] e Power [35]. No trabalho de Power foi demonstrado que se $T \in \mathcal{C}$ é um operador de Fredholm então temos $i n d(T)=w\left(\sigma_{T}\right)$, este resultado estava apenas implícito em [12]. Damos aqui uma apresentação detalhada e auto-contida dos resultados de [12, 35], usando a linguagem da K-teoria de álgebra de operadores.

Os isomorfismos (1) e (2) induzem uma sequência exata curta de $C^{*}$ - álgebras

$$
0 \longrightarrow C\left(S^{1} \times\{-1,1\}\right) \otimes \mathcal{K}_{\mathbb{Z}} \longrightarrow \mathcal{A} / \mathcal{K}_{\mathbb{R}} \longrightarrow C(X \times\{-\infty,+\infty\}) \longrightarrow 0 .
$$

No Capítulo 3 calculamos explicitamente os "connecting mappings" da sequência exata cíclica de seis termos associada a (3).

A composição do isomorfismo (1) com a projeção canônica (no quociente) pode ser estendida a um $*$-homomorfismo, que chamamos $\gamma$, da álgebra toda para o conjunto das funções contínuas de $S^{1} \times\{-1,1\}$, tomando valores em $\mathcal{L}\left(L^{2}(\mathbb{Z})\right)$. No Capítulo 4 , calculamos precisamente a imagem de $\gamma$ e verificamos que a mesma tem estrutura de produto cruzado. Sua K-teoria pôde então ser calculada pela sequência exata cíclica de Pimsner-Voiculescu [34]. Obtivemos então uma nova sequência exata curta de $C^{*}$ álgebras,

$$
0 \longrightarrow J_{0} / \mathcal{K}_{\mathbb{R}} \longrightarrow \mathcal{A} / \mathcal{K}_{\mathbb{R}} \longrightarrow \operatorname{Im} \gamma \longrightarrow 0
$$

onde $J_{0}$ é a $C^{*}$ - subálgebra de $\mathcal{L}\left(L^{2}(\mathbb{R})\right)$ gerada por todos os operadores da forma 
$a(M) b(D)$, onde $a \in C_{0}(\mathbb{R})$ e $b \in C S(\mathbb{R})$. Calculamos explicitamente os "connecting mappings" da sequência exata cíclica de seis termos associada a (4).

Os resultados obtidos com os cálculos dos "connecting mappings" das sequências exatas cíclicas de seis termos associadas a (3) e (4) nos levaram ao cálculo da K-teoria de $\mathcal{A}$ e a uma descrição explícita da fórmula do índice de Fredholm ind $: K_{1}\left(\mathcal{A} / \mathcal{K}_{\mathbb{R}}\right) \longrightarrow \mathbb{Z}$.

Seja

$$
A=\sum_{j=1}^{N} a_{j}(M) b_{j}(D),
$$

onde $a_{1}, \ldots, a_{N}$ pertencem à álgebra finitamente gerada pelas funções infinitamente diferenciáveis de período $2 \pi$ e por $s(x)=x\left(1+x^{2}\right)^{-1 / 2}$ e $b_{1}, \ldots, b_{N}$ estão na álgebra finitamente gerada por $\{s, 1\}$. Temos que $A$ é um operador pseudodiferencial clássico (isto é, com símbolo poli-homogêneo, veja [18]). As funções $a_{1}, \ldots, a_{N}$ são chamadas de semiperiódicas. A álgebra gerada pelos operadores do tipo (5) é densa em $\mathcal{A}$. Logo $\mathcal{A}$ também pode ser vista como uma $C^{*}$ - álgebra gerada por uma certa classe de operadores pseudodiferenciais com símbolos semiperiódicos.

Os resultados descritos acima sobre a estrutura de $\mathcal{A}$ foram estendidos em [23] para uma $C^{*}$-álgebra, $\mathcal{B}$, gerada por operadores pseudodiferenciais em variedades riemannianas da forma $B \times \mathbb{R}$, onde $B$ é uma variedade compacta n-dimensional.

Aparentemente para a álgebra $\mathcal{B}$ a $\operatorname{Im} \gamma$ não apresenta estrutura de produto cruzado. Sendo assim, não basta utilizar os recursos descritos no Capítulo 4 para calcular sua Kteoria. Para utilizarmos a estratégia do Capítulo 3, precisamos conhecer explicitamente alguns elementos "básicos" dos grupos $K_{i}(\mathcal{B} / \mathcal{E})$ e $K_{i}(\mathcal{E} / \mathcal{K}), i=0,1$, como por exemplo seus geradores. Estas informações parecem ser bem mais difíceis de obter nessa $C^{*}$ álgebra.

Para as álgebras $\mathcal{A}$ e $\mathcal{B}$ temos a seguinte sequência de composição (no sentido de [14], 4.3.2)

$$
\begin{gathered}
0 \subset \mathcal{K}_{\mathbb{R}} \subset \mathcal{E} \subset \mathcal{A}, \\
0 \subset \mathcal{K}_{\mathbb{R}} \otimes \mathcal{K}_{B} \subset \mathcal{E} \subset \mathcal{B} .
\end{gathered}
$$

Muitas outras $C^{*}$ - álgebras geradas por operadores pseudodiferenciais apresentam sequência de composição como acima. Por exemplo, para a $C^{*}$ - álgebra $\mathcal{U}_{M}$ gerada pelos operadores 
b-pseudodiferenciais de Melrose numa variedade compacta com bordo , Lauter [20] achou a sequência de composição

$$
0 \subset \mathcal{K}_{\mathbb{R}} \subset \mathcal{E} \subset \mathcal{U}_{M}
$$

com $\mathcal{U}_{M} / \mathcal{E}$ isomorfo (via símbolo principal) a uma álgebra de funções contínuas e $\mathcal{E} / \mathcal{K}_{\mathbb{R}}$ isomorfo à soma direta (indexada pelas componentes conexas do bordo) da álgebra de funções contínuas de $\mathbb{R}$ com valores em operadores compactos. Para mais exemplos ver $[10,24,26,27]$.

Em 1963, M. Atiyah e I. Singer em [1] publicaram a primeira prova do teorema, que posteriormente passou a chamar-se Teorema de Atiyah-Singer, que expressa o índice de Fredholm de um operador elíptico somente em termos topológicos. Outras provas podem ser encontradas em $[2,3,5,16]$.

Em 1995. R. Nest e B .Tsygan em [29, 30, 31] publicaram generalizações do Teorema de Atiyah-Singer em um contexto mais algébrico. Para essas generalizações eles utilizaram invariantes algébricos como cohomologia cíclica, K-teoria e caráter de Chern.

V. Nistor em 1997, apresentou em [32] uma nova prova do Teorema do índice de Connes-Moscovici para recobrimento [8] usando apenas invariantes algébricos.

Desde então o interesse pelo estudo dos invariantes algébricos para álgebras de operadores pseudodiferenciais tem crescido, veja por exemplo, [21, 22, 24, 25].

Temos também que álgebras de operadores pseudodiferenciais são álgebras não-comutativas naturalmente associadas a situações geométricas singulares, e existem boas razões para esperar que o estudo de invariantes dessas álgebras não-comutativas revelem informações sobre a geometria e ajudem a entender o que vem a ser uma variedade nãocomutativa [7]. 


\section{Capítulo 1}

\section{A álgebra $\mathcal{A}$}

Neste capítulo estudaremos uma subálgebra de $\mathcal{L}\left(L^{2}(\mathbb{R})\right)$, que chamamos $\mathcal{A}$, obtida como o fecho da álgebra gerada por:

i) operadores de multiplicação por funções em $C S(\mathbb{R})$.

ii) operadores de multiplicação por $e^{i j t}, j \in \mathbb{Z}$.

iii) operadores da forma $b(D):=F^{-1} b(M) F, b \in C S(\mathbb{R})$ e $F$ denotando a transformada de Fourier.

É fácil verificar que $\mathcal{A}$ é invariante por adjunto, isto é, dado $T \in \mathcal{A}$ temos $T^{*} \in \mathcal{A}$. Portanto $\mathcal{A}$ é uma $*$ - subálgebra de $\mathcal{L}\left(L^{2}(\mathbb{R})\right)$. Como $\mathcal{A}$ é fechada e tem unidade temos que $\mathcal{A}$ é uma $C^{*}$ - álgebra com unidade.

Seja $\mathcal{E}$ o ideal comutador de $\mathcal{A}$, isto é, $\mathcal{E}$ é o ideal fechado gerado pelos comutadores $[a, b]=a b-b a, a, b \in \mathcal{A}$. É fácil verificar que $\mathcal{A} / \mathcal{E}$ é abeliano. Seja $M_{A}$ o espectro da $C^{*}-$ álgebra $\mathcal{A} / \mathcal{E}$, isto é, o conjunto dos homomorfismos não nulos de $\mathcal{A} / \mathcal{E}$ em $\mathbb{C}$ munido da topologia fraca-*. Pelo Teorema 2.1.10, pág. 41 de [28] temos que $\varphi: \mathcal{A} / \mathcal{E} \longrightarrow C\left(M_{A}\right)$ onde $a \longmapsto \hat{a}$ e $\hat{a}(\phi)=\phi(a)$, para todo $a \in \mathcal{A} / \mathcal{E}$ e $\phi \in M_{A}$, é um *-isomorfismo isométrico. $\mathrm{O}$ isomorfismo $\varphi$ é chamado de isomorfismo de Gelfand.

No Teorema 1.17 apresentaremos uma fórmula explícita para $\varphi: \mathcal{A} / \mathcal{E} \longrightarrow C\left(M_{A}\right)$. Verificaremos no Lema 1.6 que $\mathcal{K}_{\mathbb{R}} \subset \mathcal{E}$ e na Proposição 1.24 estabeleceremos um isomorfismo $\psi: \mathcal{E} / \mathcal{K}_{\mathbb{R}} \longrightarrow C\left(S^{1} \times\{-1,1\}, \mathcal{K}_{\mathbb{Z}}\right)$. Estes isomorfismos nos auxiliarão, no Capítulo 3 , no cálculo da $\mathrm{K}$ - teoria de $\mathcal{A}$. 
Vamos considerar também a $C^{*}$ - álgebra conjugada $\hat{\mathcal{A}}=F^{-1} \mathcal{A} F$. Os geradores de $\hat{\mathcal{A}}$ correspondentes a i), ii) e iii) são dados por:

i) operadores da forma $a(D), a \in C S(\mathbb{R})$.

îi) operadores translação $T_{j}$, onde $T_{j}(u)(\tau):=u(\tau+j)$ com $u \in L^{2}(\mathbb{R}), j \in \mathbb{Z}$.

îî) operadores de multiplicação por funções em $C S(\mathbb{R})$.

\subsection{Ideal comutador}

O principal resultado desta seção é obtido na Proposição 1.7 onde mostraremos que o ideal comutador, $\hat{\mathcal{E}}=F^{-1} \mathcal{E} F$, da álgebra $\hat{\mathcal{A}}$ coincide com o fecho do conjunto:

$$
\left\{\sum_{j=-N}^{N} b_{j}(D) a_{j}(M) T_{j}+K ; N \in \mathbb{N}, b_{j} \in C S(\mathbb{R}), a_{j} \in C_{0}(\mathbb{R}), K \in \mathcal{K}_{\mathbb{R}}\right\} .
$$

Logo podemos concluir que $\mathcal{E}$ coincide com o fecho do conjunto:

$$
\left\{\sum_{j=-N}^{N} b_{j}(M) a_{j}(D) e^{i j M}+K ; N \in \mathbb{N}, b_{j} \in C S(\mathbb{R}), a_{j} \in C_{0}(\mathbb{R}), K \in \mathcal{K}_{\mathbb{R}}\right\} .
$$

Temos que o comutador $\left[b(D), T_{j}\right]$ é igual a zero e é conhecido que

$$
[a(M), b(D)] \in \mathcal{K}_{\mathbb{R}}, \quad \text { para } \quad a, b \in C S(\mathbb{R})
$$

(veja por exemplo [10], capítulo III, Lema 9.4). E temos que

$$
\left[T_{j}, a(M)\right]=(a(M+j)-a(M)) T_{j} \quad \text { para } \quad a \in C S(\mathbb{R}) \quad \text { e } \quad j \in \mathbb{Z} .
$$

Definição 1.1 Seja $H$ um espaço de Hilbert. Uma $C^{*}$-subálgebra $A$ de $\mathcal{L}(H)$ é irredutível se os únicos subespaços vetoriais fechados de $H$ que são invariantes por $A$ são $\{0\}$ e $H$.

No Lema abaixo damos uma condição suficiente para que uma álgebra $A \subset \mathcal{L}(H)$ seja irredutível.

Lema 1.2 Se $A \subset \mathcal{L}(H)$ é uma álgebra tal que $\{a u: a \in A\}$ é denso em $H$, para todo $u \in H$ não nulo, então $A$ é irredutivel. 


\section{Demonstração:}

Seja $M \subset H$ um subespaço vetorial fechado e invariante por $A$, onde $M \neq\{0\}$. Vamos provar que $M^{\perp}=\{0\}$, pois como $H=M \oplus M^{\perp}$ teremos $M=H$.

Sejam $x \in M^{\perp}$ e $0 \neq y \in M$. Como $\{a y: a \in A\}$ é denso em $H$ temos que existem $a_{j} \in A$ tais que $a_{j} y \rightarrow x$ e $a_{j} y \perp x$. Então $<x, x>=<\lim _{j \rightarrow \infty} a_{j} y, x>=\lim _{j \rightarrow \infty}<a_{j} y, x>=0$.

Logo $x=0$ o que implica que $M^{\perp}=\{0\}$. Portanto $A$ é irredutível.

Vamos verificar que o ideal comutador $\mathcal{E}$ da $C^{*}$ - álgebra $\mathcal{A}$ é irredutível e então utilizando o Teorema abaixo teremos $\mathcal{K}_{\mathbb{R}} \subset \mathcal{E}$.

Teorema 1.3 Seja $A$ uma $C^{*}$ - subálgebra de $\mathcal{L}(H)$ irredutivel que tem interseção não nula com $K(H)$. Então $K(H) \subset A$.

Demonstração: Ver [28], pág. 58.

Enunciaremos agora o Teorema de Stone -Weierstrass (caso complexo) que será utilizado na demonstração do próximo resultado.

Teorema 1.4 Seja B uma subálgebra de $C(X), X$ Hausdorff compacto, com a propriedade de que se $f \in B$ então $\bar{f} \in B$. Se $B$ é fechada na norma do supremo, $\|\cdot\|_{\infty}$, e separa pontos (isto é, dados $x \neq y$ em $X$, existe $f \in B$ tal que $f(x) \neq f(y)$ ), então $B=C(X)$ ou $B=\{f \in C(X): f(x)=0\}$ para algum $x$ fixado.

Demonstração: Ver [36], pág. 266.

Lema 1.5 Seja $B$ a *álgebra gerada por $\left\{a(x+j)-a(x): a \in C_{0}(\mathbb{R}), j \in \mathbb{Z}\right\}$ então $B$ é densa em $C_{0}(\mathbb{R})$.

\section{Demonstração:}

Como $\mathbb{R}$ não é compacto, para utilizarmos o Teorema 1.4 vamos considerar $X$ a compactificação de $\mathbb{R}$ por um ponto, isto é, $X=\mathbb{R} \cup\{\infty\}$. Temos que $B$ é uma subálgebra de $C(X)$ e é evidente que se $f \in B$ então $\bar{f} \in B$.

Sejam $a(x)=\frac{1}{1+x^{2}}$ e $z, y \in \mathbb{R}$ com $z \neq y$. Então para algum $j \in \mathbb{Z}, a(z+j)-a(z) \neq$ $a(y+j)-a(y)$. Consideremos a mesma $a, z=\infty$ e $y \in \mathbb{R}$ temos que $a(z+1)-a(z)=0$ e 
$a(y+1)-a(y) \neq 0$ se $y \neq-1 / 2$ e $a(y+2)-a(y) \neq 0$ se $y=-1 / 2$. Logo $B$ separa pontos de $X$, então pelo Teorema de Stone-Weierstrass temos que $\bar{B}=\{a \in C(X): a(+\infty)=$ $0\}=C_{0}(\mathbb{R})$.

Seja $T: C S(\mathbb{R}) \longrightarrow \mathcal{L}\left(L^{2}(\mathbb{R})\right)$ definida por $T(a)=a(M) . T$ é um $*$-homomorfismo injetivo, logo pelo Teorema 3.1.5 de [28] temos que $T$ é uma $*$-isometria. Utilizando esta *-isometria temos que a $*$-álgebra gerada por $\left\{a(M+j)-a(M): a \in C_{0}(\mathbb{R}), j \in \mathbb{Z}\right\}$ é densa em $\left\{b(M): b \in C_{0}(\mathbb{R})\right\}$.

Durante este trabalho estaremos muitas vezes identificando a função $b \in C S(\mathbb{R})$ com o operador $b(M)$ através desta $*$-isometria.

Lema 1.6 $O$ ideal comutador $\mathcal{E} d a C^{*}$-álgebra $\mathcal{A}$ é irredutível.

\section{Demonstração:}

Consideremos $\eta(x)=\frac{1}{1+x^{2}}, x \in \mathbb{R}$. É conhecido que

$$
F^{-1} \eta(x)=\frac{1}{\sqrt{4 \pi}} \int_{0}^{+\infty} \frac{e^{-\delta}}{\sqrt{\delta}} e^{\frac{-|x|^{2}}{4 \delta}} d \delta
$$

(veja, por exemplo, [36] pág. 128).

Temos que $\left|F^{-1} \eta(x)\right| \leq \frac{1}{\sqrt{4 \pi}} \int_{0}^{+\infty} \frac{e^{-\delta}}{\sqrt{\delta}} d \delta$ e sabemos que

$$
\begin{aligned}
& \int_{0}^{1} \frac{e^{-\delta}}{\sqrt{\delta}} d \delta<\int_{0}^{1} \frac{1}{\sqrt{\delta}} d \delta<\infty \\
& \int_{1}^{+\infty} \frac{e^{-\delta}}{\sqrt{\delta}} d \delta \leq \int_{1}^{+\infty} e^{-\delta} d \delta<\infty .
\end{aligned}
$$

Logo por (1.3) e (1.4) temos que $F^{-1} \eta$ é limitada. Fazendo $u=\frac{x}{2 \sqrt{\delta}}$ temos $d u=\frac{d x}{2 \sqrt{\delta}}$, $\log 0$

$$
\int_{\mathbb{R}} F^{-1} \eta(x) d x=\frac{2}{\sqrt{4 \pi}} \int_{0}^{+\infty} e^{-\delta} \int_{-\infty}^{+\infty} e^{-u^{2}} d u d \delta<\infty
$$

o que implica que $F^{-1} \eta \in L^{1}(\mathbb{R})$.

Sejam $\varphi \in \mathcal{S}(\mathbb{R})$ e $T=F^{-1} \eta$. Temos que $\eta(D)(\varphi)=F^{-1}(\hat{\varphi} \hat{T})$ e como $T$ é uma distribuição temperada pelo Teorema IX.4 de [36] sabemos que $F^{-1}(\hat{\varphi} \hat{T})=\frac{1}{\sqrt{2 \pi}} T * \varphi$. Como $T \in L^{1}(\mathbb{R})$ e $\varphi \in \mathcal{S}(\mathbb{R}) \subset L^{1}(\mathbb{R})$ podemos escrever

$$
\frac{1}{\sqrt{2 \pi}} T * \varphi(x)=\frac{1}{\sqrt{2 \pi}} \int_{\mathbb{R}} T(x-y) \varphi(y) d y \text { para todo } x \in \mathbb{R} .
$$


Logo $\eta(D)$ é um operador integral com núcleo $T(x-y)>0$. Tomemos $0 \neq u \in L^{2}(\mathbb{R})$ e $\psi \in C_{c}^{\infty}(\mathbb{R})$ tal que $\psi u \neq 0$. Temos que $\eta(D) \psi u \neq 0$, já que $\eta(M)$ é injetor, e vamos verificar agora que $\eta(D) \psi u$ é contínua.

Sabemos que para todo $x \in \mathbb{R}, \eta(D) \psi u(x)=\int_{\mathbb{R}} T(x-y)(\psi u)(y) d y$. Já verificamos que $T \in L^{1}(\mathbb{R})$ e é limitada. Por Holder temos que $\psi u \in L^{1}(\mathbb{R})$ e como $|T(x-y)(\psi u)(y)| \leq$ $C|(\psi u)(y)|$ segue que $T(x-y)(\psi u)(y)$ é limitada por uma função em $L^{1}(\mathbb{R})$, independente de $x$. Então pelo Teorema da Convergência Dominada temos que para cada $x_{0} \in \mathbb{R}$ $\lim _{x \rightarrow x_{0}} \int_{\mathbb{R}} T(x-y)(\psi u)(y) d y=\int_{\mathbb{R}^{x \rightarrow x_{0}}} \lim _{x} T(x-y)(\psi u)(y) d y$.

Usando novamente o Teorema da Convergência Dominada temos que $\lim _{v \rightarrow v_{0}} T(v)=$

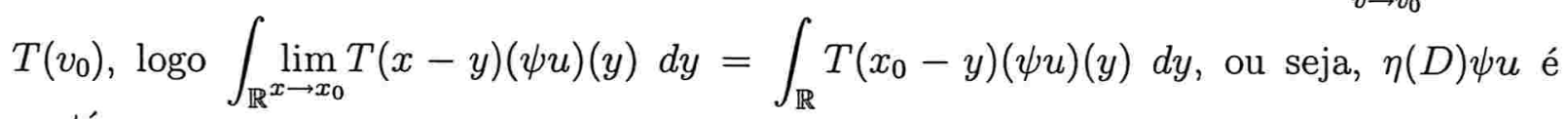
contínua.

Como $\eta(D) \psi u \neq 0$ temos que existe $x_{0} \in \mathbb{R}$ tal que $\eta(D) \psi u\left(x_{0}\right) \neq 0$. Então temos que $\operatorname{Re}\left(\eta(D) \psi u\left(x_{0}\right)\right) \neq 0$ ou $\operatorname{Im}\left(\eta(D) \psi u\left(x_{0}\right)\right) \neq 0$ ou ambas o são.

Suponhamos que $\operatorname{Re}\left(\eta(D) \psi u\left(x_{0}\right)\right) \neq 0$. Então temos que $\operatorname{Re}\left(\eta(D) \psi u\left(x_{0}\right)\right)>0$ ou $\operatorname{Re}\left(\eta(D) \psi u\left(x_{0}\right)\right)<0$. Se $\operatorname{Re}\left(\eta(D) \psi u\left(x_{0}\right)\right)>0$ então pela continuidade de $\eta(D) \psi u$ temos que $\operatorname{Re}(\eta(D) \psi u)>0$ em um aberto $B \subset \mathbb{R}$ contendo $x_{0}$. Pelo Corolário I.2.2 de [19] existe $\varphi \in C_{c}^{\infty}(B)$ tal que $0 \leq \varphi \leq 1$ e $\varphi \equiv 1$ em um aberto contido em $B$.

Portanto $\operatorname{Re}(\varphi \eta(D) \psi u)(x) \geq 0$ para todo $x \in \mathbb{R}$. E como $\eta(D)$ tem núcleo positivo $\operatorname{Re}(\eta(D) \varphi \eta(D) \psi u)(x)>0$ para todo $x \in \mathbb{R}$.

Se $\operatorname{Re}\left(\eta(D) \psi u\left(x_{0}\right)\right)<0$ devemos proceder como no caso da $\operatorname{Re}\left(\eta(D) \psi u\left(x_{0}\right)\right)>0$. Se $\operatorname{Im}\left(\eta(D) \psi u\left(x_{0}\right)\right) \neq 0$ é análogo ao caso da parte real ser diferente de zero. Portanto existe $\varphi \in C_{c}^{\infty}(B)$ tal que $[\eta(D) \varphi \eta(D) \psi u](x) \neq 0$ para todo $x \in \mathbb{R}$.

Dado $v \in C_{c}^{\infty}(\mathbb{R})$ seja $w=\frac{v}{\eta(D) \varphi(M) \eta(D) \psi(M) u}$. Temos que

$$
v=w(M) \eta(D) \varphi(M) \eta(D) \psi(M) u .
$$

Como $w(M), \varphi(M), \psi(M) \in \mathcal{A}$ e no Lema 1.5 vimos que $\eta(D) \in \mathcal{E}$ temos que $v \in\{E u: E \in \mathcal{E}\}$ o que implica que $C_{c}^{\infty}(\mathbb{R}) \subset\{E u: E \in \mathcal{E}\}$.

Usando que $C_{c}^{\infty}(\mathbb{R})$ é denso em $L^{2}(\mathbb{R})$ temos que $\{E u: E \in \mathcal{E}\}$ é denso em $L^{2}(\mathbb{R})$, para todo $u \neq 0$. Então pelo Lema 1.2 podemos concluir que $\mathcal{E}$ é irredutível. 
Temos que $[a(M), b(D)] \in \mathcal{K}_{\mathbb{R}} \cap \mathcal{E}$, para $a, b \in C S(\mathbb{R})$, e vamos verificar agora que $[a(M), b(D)] \neq 0$ para algum $a, b \in C S(\mathbb{R})$. Vamos verificar mais, que $a$ e $b$ podem ser tomados em $\mathcal{S}(\mathbb{R})$.

Observação $1.61 / 2:[a(M), b(D)] \neq 0$ para algum $a, b \in \mathcal{S}(\mathbb{R})$.

De fato, sejam $a, b \in \mathcal{S}(\mathbb{R})$, é fácil verificar que

$$
[a(M), b(D)] u(x)=\sqrt{2 \pi} \int_{\mathbb{R}}\left((a(x)-a(y)) F^{-1} b(x-y)\right) u(y) d y .
$$

Logo $[a(M), b(D)]$ é um operador integral com núcleo $K(x, y)=(a(x)-a(y)) F^{-1} b(x-y)$. Seja $T_{K}=[a(M), b(D)]$, afirmamos que $\|K\|_{L^{2}} \neq 0$ implica $T_{K} \neq 0$.

De fato, pelo Teorema VI.23 de [36] temos que a norma Hilbert-Schmidt $\left\|T_{K}\right\|_{H S}=$ $\|K\|_{L^{2}}, \operatorname{logo} K=0$ se, e só se, $T_{K}=0$.

Consideremos então $b(x)=e^{-x^{2}}$ e $a$ não constante. Temos que $F^{-1} b(x-y)=$ $\frac{\sqrt{2}}{2} e^{-(x-y)^{2} / 4}>0$ para todo $(x, y) \in \mathbb{R}^{2} \operatorname{logo}\|K(x, y)\|_{L^{2}} \neq 0$. Segue da afirmação que $T_{K} \neq 0$.

Portanto pelo Teorema 1.3 temos $\mathcal{K}_{\mathbb{R}} \subset \mathcal{E}$.

Proposição 1.7 O ideal comutador $\hat{\mathcal{E}} d a C^{*}$ - álgebra conjugada $\hat{\mathcal{A}}$ coincide com o fecho de

$$
\hat{\mathcal{E}}_{A}:=\left\{\sum_{j=-N}^{N} b_{j}(D) a_{j}(M) T_{j}+K ; N \in \mathbb{N}, b_{j} \in C S(\mathbb{R}), a_{j} \in C_{0}(\mathbb{R}), K \in \mathcal{K}_{\mathbb{R}}\right\}
$$

\section{Demonstração:}

Como já foi provado temos que $\mathcal{K}_{\mathbb{R}} \subset \mathcal{E}$, logo

$$
\mathcal{K}_{\mathbb{R}} \subset \hat{\mathcal{E}} .
$$

Por (1.2) temos que todos operadores da forma $b(D)(a(M+j)-a(M)) T_{j}$ estão em $\hat{\mathcal{E}}$ para $b \in C S(\mathbb{R}), j \in \mathbb{Z}$ e $a \in C_{0}(\mathbb{R})$. Assim, pelo Lema 1.5 temos $\hat{\mathcal{E}}_{A} \subset \hat{\mathcal{E}}$.

Por outro lado, usando (1.1), (1.2), e (1.5), temos que $\hat{\mathcal{E}}_{A}$ é uma subálgebra de $\hat{\mathcal{A}}$ que contém os comutadores de todos os geradores de $\hat{\mathcal{A}}$ listados no começo do capítulo. Além disso, $\hat{\mathcal{E}}_{A}$ é invariante a direita ou a esquerda por multiplicações por estes operadores. Tomando limite, segue que o fecho de $\hat{\mathcal{E}}_{A}$ é um ideal de $\hat{\mathcal{A}}$ que contém todos os seus comutadores. Logo, $\hat{\mathcal{E}}$ está contido no fecho de $\hat{\mathcal{E}}_{A}$. 


\subsection{A álgebra $\mathcal{A}^{\sharp}$}

Nesta seção estudaremos $\mathcal{A}^{\sharp}$ a $C^{*}$ - álgebra abeliana gerada pelos operadores do tipo i) e ii) da página 17. No Lema 1.10 explicitaremos o espectro, $M_{\sharp}$, de $\mathcal{A}^{\sharp}$.

Seja $\mathcal{A}_{0}^{\sharp}$ a álgebra finitamente gerada por $C S(\mathbb{R})$ e $P_{2 \pi}$. Sejam $\chi_{+}$e $\chi_{-} \in C S(\mathbb{R})$ tais que $\chi_{ \pm}( \pm \infty)=1, \chi_{+}+\chi_{-}=1$ e $\chi_{ \pm}(t)=0$ se $\mp t>1$. É fácil verificar que todo $a \in \mathcal{A}_{0}^{\sharp}$ é da forma

$$
a=a_{+} \chi_{+}+a_{-} \chi_{-}+a_{0}, a_{ \pm} \in P_{2 \pi}, a_{0} \in C_{0}(\mathbb{R})
$$

onde a escolha de $a_{+}, a_{-}$e $a_{0}$ é única. Vamos provar que $\mathcal{A}_{0}^{\sharp}$ é fechada e assim teremos $\mathcal{A}^{\sharp}=\mathcal{A}_{0}^{\sharp}$.

Seja $f_{n}=a_{n} \chi_{+}+b_{n} \chi_{-}+c_{n} \in \mathcal{A}_{0}^{\sharp}$ e consideremos a sequência $\left(f_{n}\right)_{n}$ convergindo uniformemente para $d$. Vamos provar que $d \in \mathcal{A}_{0}^{\sharp}$.

Para cada $y>10$ temos que $\left\|a_{n}\right\|_{\infty}=\sup _{x \in[y, y+2 \pi]}\left|a_{n}(x)\right| \leq \sup _{x \in[y, y+2 \pi]}\left|a_{n}(x)+c_{n}(x)\right|+$ $\sup _{x \in[y, y+2 \pi]}\left|c_{n}(x)\right| \leq \sup _{x \geq 1}\left|a_{n}(x)+c_{n}(x)\right|+\sup _{x \in[y, y+2 \pi]}\left|c_{n}(x)\right|$. Fazendo $y \rightarrow \infty$ temos que $\left\|a_{n}\right\|_{\infty} \leq \sup _{x \geq 1}\left|a_{n}(x)+c_{n}(x)\right| \leq\left\|f_{n}\right\|_{\infty}$

Com isto temos que $\left(a_{n}\right)_{n}$ é Cauchy e pelo mesmo argumento $\left(b_{n}\right)_{n}$ é Cauchy. Digamos que $a_{n} \rightarrow a$ e $b_{n} \rightarrow b, a, b \in P_{2 \pi}$. Temos então que $d-a \chi_{+}-b \chi_{-}=\lim c_{n} \in C_{0}(\mathbb{R}), \operatorname{logo}$ $d \in \mathcal{A}_{0}^{\sharp}$. Portanto $\mathcal{A}_{0}^{\sharp}$ é fechada. Provamos então que:

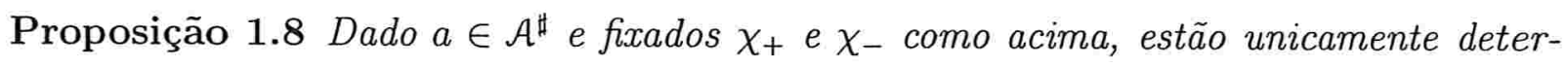
minados $a_{0} \in C_{0}(\mathbb{R})$ e $a_{ \pm} \in P_{2 \pi}$ tais que $a=a_{+} \chi_{+}+a_{-} \chi_{-}+a_{0}$.

Vamos achar o espectro $M_{\sharp}$ da $C^{*}$ - álgebra $\mathcal{A}^{\sharp}$. Para isto vamos definir duas classes de funcionais lineares multiplicativos.

1) A cada $t \in \mathbb{R}$ vamos definir

$$
\begin{aligned}
w_{t}: & \mathcal{A}^{\sharp} \longrightarrow \mathbb{C} \\
f & \longmapsto f(t)
\end{aligned}
$$

2) Para $\theta \in \mathbb{R}$,

$$
w_{\theta,+}: \mathcal{A}^{\sharp} \longrightarrow \mathbb{C}
$$




$$
\begin{aligned}
f & \longmapsto \lim _{k \rightarrow \infty} f(\theta+2 \pi k):=f_{\theta}^{+} \\
w_{\theta,-}: \mathcal{A}^{\sharp} & \longmapsto \mathbb{C} \\
f & \longmapsto \lim _{k \rightarrow \infty} f(\theta-2 \pi k):=f_{\theta}^{-}
\end{aligned}
$$

Usando que para $f \in \mathcal{A}^{\sharp}$ temos $f=f_{+} \chi_{+}+f_{-} \chi_{-}+f_{0} \operatorname{com} f_{ \pm} \in P_{2 \pi}$ e $f_{0} \in C_{0}(\mathbb{R})$, é fácil verificar que os limites existem e que $\mathbb{R} \ni \theta \longmapsto f_{\theta}^{ \pm} \in \mathbb{C}$ são funções $2 \pi$-periódicas.

Proposição 1.9 Os funcionais $w_{t}$ e $w_{\theta, \pm}, t, \theta \in \mathbb{R}$ são todos os funcionais lineares multiplicativos de $\mathcal{A}^{\sharp}$. E temos que $\mathbb{R}$ é denso em $M_{\sharp}$.

\section{Demonstração:}

Temos que $\mathbb{R} \subset M_{\sharp}$, pois basta considerarmos a identificação

$$
\begin{aligned}
i: \mathbb{R} & \longrightarrow M_{\sharp} \\
t & \longmapsto w_{t} .
\end{aligned}
$$

Vamos verificar que $i$ é contínua e injetora.

$\triangleright$ injetividade

Sejam $t, s \in \mathbb{R}$ com $t \neq s$ e $\varphi \in C S(\mathbb{R})$ tal que $\varphi(t) \neq 0$ e $\varphi(s)=0$. Temos que $\varphi \in \mathcal{A}^{\sharp} \mathrm{e}$ $w_{t}(\varphi) \neq w_{s}(\varphi)$, isto é, $i(s) \neq i(t)$.

$\triangleright$ continuidade

Seja $\left\{t_{\alpha}\right\} \in \mathbb{R}$ uma rede com $t_{\alpha} \rightarrow t$. Queremos mostrar que $i\left(t_{\alpha}\right) \rightarrow i(t)$.

Temos que $\varphi\left(t_{\alpha}\right) \rightarrow \varphi(t)$ para todo $\varphi \in \mathcal{A}^{\sharp}$. Portanto $w_{t_{\alpha}}(\varphi) \rightarrow w_{t}(\varphi)$ para todo $\varphi \in \mathcal{A}^{\sharp}, \log$ o $i$ é contínua.

Suponhamos que $w \in M_{\sharp}$ e seja $s \in C S(\mathbb{R})$ a função $s(t)=\frac{t}{\sqrt{1+t^{2}}}$.

Como $-1 \leq s \leq 1$ temos que $-1 \leq w(s) \leq 1$. Vamos provar que se $|w(s)|<1$ então $w=w_{a}$ onde $a \in \mathbb{R}$ resolve $s(a)=w(s)$.

Por Stone-Weierstrass temos que os polinômios em $s$ são densos em $C S(\mathbb{R})=$ $=C([-\infty,+\infty])$, logo $w$ e $w_{a}$ coincidem sobre $C S(\mathbb{R})$.

Seja $\chi \in C_{0}(\mathbb{R})$ satisfazendo $\chi(a)=1$, temos $\chi(M) e^{i M} \in C S(\mathbb{R})$ e então $w_{a}\left(e^{i M}\right)=$ $e^{i a}=w_{a}\left(\chi(M) e^{i M}\right)=w\left(\chi(M) e^{i M}\right)=w(\chi(M)) w\left(e^{i M}\right)=w\left(e^{i M}\right)$. Provando que $w$ e $w_{a}$ coincidem sobre o conjunto de geradores de $\mathcal{A}^{\sharp}$. 
Para o caso $w(s)= \pm 1$, seja $\theta$ tal que $w\left(e^{i M}\right)=e^{i \theta}$. Temos que $w_{\theta, \pm}(s)=\lim _{k \rightarrow \infty} s(\theta \pm$ $2 \pi k)= \pm 1$. Portanto $w_{\theta, \pm}(s)=w(s)$.

Usando novamente o fato de que os polinômios em $s$ são densos em $C S(\mathbb{R})$ temos que $w$ e $w_{\theta, \pm}$ coincidem em $C S(\mathbb{R})$.

Temos que $w_{\theta, \pm}\left(e^{i M}\right)=\lim _{k \rightarrow \infty} e^{i(\theta \pm 2 \pi k)}=e^{i \theta}$. Portanto $w\left(e^{i M}\right)=w_{\theta, \pm}\left(e^{i M}\right), \operatorname{logo} w$ e $w_{\theta, \pm}$ coincidem sobre os geradores de $\mathcal{A}^{\sharp}$.

Agora só nos falta provar que $\mathbb{R}$, ou melhor, $i(\mathbb{R})$ é denso em $M_{\sharp}$.

Seja $K=\overline{i(\mathbb{R})} \subset M_{\sharp}$ e suponhamos que $K \neq M_{\sharp}$. Então existe $w_{0} \in M_{\sharp} \backslash K$, e pelo Lema de Urysohn temos que existe $\hat{f} \in C\left(M_{\sharp}\right)$ tal que $\hat{f}\left(w_{0}\right)=1$ e $\hat{f} \equiv 0$ em $K$.

Temos que $\mathcal{A}^{\sharp}$ é isomorfo a $C\left(M_{\sharp}\right)$, pelo Teorema de Gelfand. Logo existe $f \in \mathcal{A}^{\sharp}$ tal que $\hat{f}$ é a imagem de $f$ por este isomorfismo e temos que $f(x)=w_{x}(f)=\hat{f}\left(w_{x}\right)=0$ para todo $x \in \mathbb{R}$, ou seja, $f$ é nula. Absurdo, pois $\hat{f}$ não é nula. Portanto $\mathbb{R}$ é denso em $M_{\sharp}$.

Lema 1.10 $M_{\sharp}$ é homeomorfo a um subconjunto de $[-\infty,+\infty] \times S^{1}$ a saber, $M_{\sharp} \approx$ $\left\{\left(x, e^{i x}\right): x \in \mathbb{R}\right\} \cup\left(\{-\infty,+\infty\} \times S^{1}\right)$.

\section{Demonstração:}

Pela Proposição acima temos que $M_{\sharp}=\left\{w_{t}: t \in \mathbb{R}\right\} \cup\left\{w_{\theta, \pm}: \theta \in \mathbb{R}\right\}$ com a topologia fraca-*. Usando as notações acima temos que $f_{\theta+2 \pi j}^{ \pm}=f_{\theta}^{ \pm}$, logo $f_{\theta}^{ \pm}$é um par de funções em $\mathbb{R} / 2 \pi \mathbb{Z}=S^{1}$.

Consideremos as inclusões

$$
\begin{aligned}
i_{1}: C S(\mathbb{R}) & \longrightarrow \mathcal{A}^{\sharp} \\
a & \longmapsto a(M) \\
\mathrm{e} & \\
i_{2}: P_{2 \pi} & \longrightarrow \mathcal{A}^{\sharp} \\
a & \longmapsto a(M) .
\end{aligned}
$$

Temos que o espectro de $C S(\mathbb{R})$ é o conjunto $[-\infty,+\infty]$ e que o espectro de $P_{2 \pi}$ é $S^{1}$. Consideremos

$$
i_{1}^{*}: M_{\sharp} \longrightarrow[-\infty,+\infty],
$$


onde $i_{1}^{*}(w)=w \circ i_{1}$, e também

$$
i_{2}^{*}: M_{\sharp} \longrightarrow S^{1},
$$

onde $i_{2}^{*}(w)=w \circ i_{2}$. As aplicações $i_{1}^{*}$ e $i_{2}^{*}$ são chamadas de aplicações duais de $i_{1}$ e $i_{2}$ respectivamente.

Seja $j: M_{\sharp} \longrightarrow[-\infty,+\infty] \times S^{1}$ dada por $j(w)=\left(i_{1}^{*}(w), i_{2}^{*}(w)\right)$ para $w \in M_{\sharp}$.

Como $\operatorname{Im} i_{1}$ e $\operatorname{Im} i_{2}$ geram $\mathcal{A}^{\sharp}$ é fácil ver que $j$ é injetora. Como $M_{\sharp}$ é compacto (Teorema 1.3.5, [28]), $[-\infty,+\infty] \times S^{1}$ é Hausdorff e $j$ é contínua temos que $j$ é um homeomorfismo sobre a imagem. Podemos então identificar topologicamente $M_{\sharp}$ com um subconjunto de $[-\infty,+\infty] \times S^{1}$. Pela definição da aplicação dual, podemos concluir que $M_{\sharp}=\left\{\left(x, e^{i x}\right): x \in \mathbb{R}\right\} \cup\left(\{-\infty,+\infty\} \times S^{1}\right)$.

Pelo Teorema de Gelfand, temos que $\varphi: \mathcal{A}^{\sharp} \rightarrow C\left(M_{\sharp}\right)$ definido por $\varphi(a)=\hat{a}$ é um *-isomorfismo isométrico. Mas sabemos quem são os funcionais de $M_{\sharp}$, logo temos

$$
\begin{aligned}
& \hat{a}\left(w_{t}\right)=w_{t}(a)=a(t), t \in \mathbb{R} \quad \mathrm{e} \\
& \hat{a}\left(w_{\theta, \pm}\right)=w_{\theta, \pm}(a)=a_{\theta}^{ \pm}, e_{ \pm}^{i \theta} \in S^{1} .
\end{aligned}
$$

\subsection{A álgebra $J_{0}$}

Nesta seção estudaremos $J_{0} \subset \mathcal{L}\left(L^{2}(\mathbb{R})\right)$ a $C^{*}$ - subálgebra gerada por $a(M) b(D)$, $a \in C_{0}(\mathbb{R})$ e $b \in C S(\mathbb{R})$. No Lema 1.15 mostraremos que $J_{0} / \mathcal{K}_{\mathbb{R}} \cong C_{0}(\mathbb{R} \times\{-\infty,+\infty\})$ e daremos uma fórmula explícita para o isomorfismo de Gelfand. E na Proposição 1.16 vamos verificar que $J_{0} \cap \mathcal{E}=\mathcal{K}_{\mathbb{R}}$. Os resultados desta seção serão utilizados na próxima seção e também no Capítulo 4.

Definição $1.11 O$ espaço símbolo $M_{A}$ de uma $C^{*}$-álgebra $A$ é o espectro da álgebra $A / E$, onde $E$ é o ideal comutador de A.

Definição $1.12 O \sigma$-símbolo de uma $C^{*}$ - álgebra $A$ é a composição da projeção canônica $\pi$ com a transformada de Gelfand da $C^{*}$ - álgebra $A / E$, onde $E$ é o ideal comutador. Notação: $\sigma: A \rightarrow C\left(M_{A}\right)$ com $a \mapsto \sigma_{a}=\widehat{\pi(a)}$. 
Lema 1.13 $A C^{*}$ - álgebra $J_{0}$ é irredutível.

\section{Demonstração:}

Vamos verificar que $\left\{a u: a \in J_{0}\right\}$ é denso em $L^{2}(\mathbb{R})$, para todo $u$ não nulo. Então pelo Lema 1.2 teremos que $J_{0}$ é irredutível.

Na demonstração do Lema 1.6, vimos que, dados $v \in C_{c}^{\infty}(\mathbb{R})$ existem $\eta, \varphi, \psi, w, u$ tais que

$$
v=w(M) \eta(D) \varphi(M) \eta(D) \psi(M) u .
$$

Temos que $w(M) \eta(D), \varphi(M) \eta(D)$ e $\psi(M) \in J_{0}$, logo $v \in\left\{a u: a \in J_{0}\right\}$. Como $v \in C_{c}^{\infty}(\mathbb{R})$ é qualquer temos que $C_{c}^{\infty}(\mathbb{R}) \subset\left\{a u: a \in J_{0}\right\}$. Portanto $\left\{a u: a \in J_{0}\right\}$ é denso em $L^{2}(\mathbb{R})$, para todo $u$ não nulo.

Lema 1.14 O comutador $[a(M), b(D)]$ é compacto para $a, b \in C_{0}(\mathbb{R})$.

\section{Demonstração:}

Consideremos $a, b \in \mathcal{S}(\mathbb{R})$. É fácil verificar que

$$
a(M) b(D) u(x)=\frac{1}{2 \pi} \int_{\mathbb{R}}\left(\int_{\mathbb{R}} e^{i t(x-y)} b(t) a(x) d t\right) u(y) d y
$$

e também que

$$
b(D) a(M) u(x)=\frac{1}{2 \pi} \int_{\mathbb{R}}\left(\int_{\mathbb{R}} e^{i t(x-y)} b(t) a(y) d t\right) u(y) d y .
$$

Logo $a(M) b(D)$ é um operador integral com núcleo $\hat{b}(y-x) a(x) \in L^{2}\left(\mathbb{R}^{2}\right)$ e $b(D) a(M)$ é um operador integral com núcleo $\hat{b}(y-x) a(y) \in L^{2}\left(\mathbb{R}^{2}\right)$. Portanto pelo Teorema VI.23 de [36] temos que $a(M) b(D)$ e $b(D) a(M)$ são compactos e assim $[a(M), b(D)]$ é compacto para $a, b \in \mathcal{S}(\mathbb{R})$.

O fim da demonstração segue do fato de $\mathcal{S}(\mathbb{R})$ ser denso em $C_{0}(\mathbb{R})$.

Observação $1.141 / 2$ : Também temos que $a(M) b(D) \in \mathcal{K}_{\mathbb{R}}$ se $a \in C_{0}(\mathbb{R})$ e $b \in$ $L^{\infty}(\mathbb{R}) \cap L^{2}(\mathbb{R})$, a demonstração é análoga a do Lema acima.

Pelo Lema 1.14 e pela Observação $1.61 / 2$ da página 22 temos que $J_{0} \cap \mathcal{K}_{\mathbb{R}} \neq 0$. Logo pelo Teorema 1.3 temos que $\mathcal{K}_{\mathbb{R}} \subset J_{0}$ e assim $\mathcal{K}_{\mathbb{R}} \subset J_{0} \cap \mathcal{E}$. 
Seja $G$ o ideal comutador de $J_{0}$. Por (1.1) temos que $G \subset \mathcal{K}_{\mathbb{R}}$ e que $G$ é não nulo pela Observação $1.61 / 2$ da página 22. Como $\mathcal{K}_{\mathbb{R}} \subset J_{0}$ segue que $G=\mathcal{K}_{\mathbb{R}}$, pois o único ideal não nulo de $\mathcal{K}_{\mathbb{R}}$ é $\mathcal{K}_{\mathbb{R}}$.

Lema $1.15 O$ espaço símbolo $M_{J} d a C^{*}$ - álgebra $J_{0}$ é dado como:

$$
M_{J}=\mathbb{R} \times\{-\infty,+\infty\} .
$$

O $\sigma$-símbolo é dado por:

$$
\begin{gathered}
\sigma_{a}(m, \pm \infty)=a(m), m \in \mathbb{R}, a \in C_{0}(\mathbb{R}) \quad e \\
\sigma_{b(D)}(m, \pm \infty)=b( \pm \infty), m \in \mathbb{R}, b \in C S(\mathbb{R}) .
\end{gathered}
$$

\section{Demonstração:}

Consideremos a aplicação

$$
\begin{aligned}
i: C_{0}(\mathbb{R}) & \longrightarrow J_{0} / \mathcal{K}_{\mathbb{R}} \\
a & \longmapsto[a(M)]_{\mathcal{K}_{\mathbb{R}}}
\end{aligned}
$$

Temos que $i$ é injetora já que $a(M) \in \mathcal{K}_{\mathbb{R}}$ se, e só se, $a \equiv 0$, logo sua aplicação dual $i^{*}: M_{J} \longrightarrow \mathbb{R}$ onde $\mathbb{R}$ é o espectro de $C_{0}(\mathbb{R})$ é sobrejetora (veja, por exemplo, [10], pág. 314).

Para $w \in M_{J}$ sejam $t_{0}=i^{*}(w), \phi \in C_{c}^{\infty}(\mathbb{R}), \phi$ real, com $\phi$ igual a um em uma vizinhança de $t_{0}$.

Sejam $A=\phi(M) s(D)$ onde $s(x)=\frac{x}{\sqrt{1+x^{2}}}$ e $\xi_{0}=\sigma_{A}(w)=w\left([\phi(M) s(D)]_{\mathcal{K}_{\mathbf{R}}}\right)$. Temos que $\xi_{0}$ é real, pois $(\phi(M) s(D))^{*}=s(D) \phi(M)$ que é côngruo módulo compacto a $\phi(M) s(D)$. Logo $[\phi(M) s(D)]_{\mathcal{K}_{\mathbb{R}}}^{*}=[\phi(M) s(D)]_{\mathcal{K}_{\mathbb{R}}}$, consequentemente $\xi_{0}=\bar{\xi}_{0}$.

Definimos assim o par $\left(t_{0}, \xi_{0}\right)$. Vamos verificar que $\xi_{0}$ não depende da $\phi$ considerada.

Sejam $\phi$ e $\psi \in C_{c}^{\infty}(\mathbb{R})$ com $\phi$ igual a um em uma vizinhança $U$ de $t_{0}$ e $\psi$ igual a um em uma vizinhança $\mathrm{V}$ de $t_{0}$. Seja $B=\psi(M) s(D)$, seja $\chi \in C_{c}^{\infty}(\mathbb{R})$ com suporte contido em $U \cap V$ e igual a um em uma vizinhança de $t_{0}$. Como $(1-\chi)(\phi-\psi)=\phi-\psi$ temos que $A-B=(1-\chi)(M)(A-B)$. Logo $\sigma_{A-B}(w)=\sigma_{A-B}(w)-\sigma_{\chi(M)(A-B)}(w)=$ $\sigma_{A-B}(w)-\sigma_{\chi(M)}(w) \sigma_{A-B}(w)=0$, pois $\sigma_{\chi(M)}(w)=\chi\left(t_{0}\right)=1$. Portanto $\sigma_{A}(w)=\sigma_{B}(w)$.

Temos que $\phi(M) s(D) \phi(M) s(D)$ é côngruo módulo compacto a $\phi^{2}(M) s^{2}(D)$ e que $\phi^{2}(M)\left(1-s^{2}\right)(D)$ é compacto pelo Lema 1.14. Logo $A^{2}$ é côngruo módulo compacto a 
$\phi^{2}(M)$, com isto temos que $\xi_{0}^{2}=w\left([A]_{\mathcal{K}_{\mathbb{R}}}^{2}\right)=w\left([\phi(M)]_{\mathcal{K}_{\mathbb{R}}}^{2}\right)=\phi^{2}\left(i^{*}(w)\right)=1$. Portanto $\xi_{0}= \pm 1$.

Definimos então

$$
\begin{aligned}
\nu: M_{J} & \longrightarrow \mathbb{R} \times\{-1,1\} \\
w & \longmapsto\left(i^{*}(w), \xi\right),
\end{aligned}
$$

onde $\xi=w\left([\phi(M) s(D)]_{\mathcal{K}_{\mathbb{R}}}\right), \phi \in C_{c}^{\infty}(\mathbb{R})$ com $\phi$ igual a um em uma vizinhança de $i^{*}(w)$.

Vamos provar agora que se $a \in C_{0}(\mathbb{R})$ e $\nu(w)=(t, \xi)$ então $w\left([a(M) s(D)]_{\mathcal{K}_{\mathbb{R}}}\right)=a(t) \xi$. De fato, seja $\chi_{j} \in C_{c}^{\infty}(\mathbb{R})$ com $\chi_{j}$ igual a um em $(-j, j)$ então $\xi=w\left(\left[\chi_{j}(M) s(D)\right]_{\mathcal{K}_{\mathbb{R}}}\right)$. Temos que $w\left(\left[\chi_{j}(M) a(M) s(D)\right]_{\mathcal{K}_{\mathbf{R}}}\right)=a(t) \xi$ para todo $j$ suficientemente grande, mas $\chi_{j}(M) a(M) s(D) \rightarrow a(M) s(D)$. Logo temos o que queríamos.

Vamos verificar que $\nu$ é um homeomorfismo.

$\triangleright$ injetora

Sejam $w, w, \in M_{J}$ tais que $\left(t_{0}, \xi_{0}\right)=\nu(w)=\nu\left(w^{\prime}\right)=(t, \xi)$. Temos então que $t_{0}=i^{*}(w)=i^{*}\left(w^{\prime}\right)=t$ e $\sigma_{\phi}(w)=\sigma_{\phi}\left(w^{\prime}\right)$ para toda $\phi \in C_{c}^{\infty}(\mathbb{R})$. Como $\xi=\xi_{0}$ temos também $\sigma_{\phi(M) s(D)}(w)=\sigma_{\phi(M) s(D)}\left(w^{\prime}\right)$, para todo $\phi \in C_{c}^{\infty}(\mathbb{R})$.

O conjunto de todos os $\phi(M) s(D)$ como acima geram $J_{0}$, módulo $\mathcal{K}_{\mathbb{R}}$, logo $\sigma_{A}(w)=$ $\sigma_{A}\left(w^{\prime}\right)$ para todo $A \in J_{0}$. Portanto $w=w^{\prime}$.

$\triangleright$ sobrejetora

Consideremos o operador paridade $P$, isto é $(P f)(x)=f(-x), f \in L^{2}(\mathbb{R})$. É fácil verificar que $P a(M) P=a(-M)$ e $P b(D) P=b(-D)$ para $a \in C_{0}(\mathbb{R})$ e $b \in C S(\mathbb{R})$.

Dado $w \in M_{J}$ vamos definir $\lambda\left([A]_{\mathcal{K}_{\mathbb{R}}}\right)=w\left([P A P]_{\mathcal{K}_{\mathbb{R}}}\right), A \in J_{0}$. Seja $\nu(w)=\left(t_{0}, \xi_{0}\right)=$ $\left(i^{*}(w), \xi_{0}\right)$ como $\lambda\left([a(M)]_{\mathcal{K}_{\mathbb{R}}}\right)=w\left([a(-M)]_{\mathcal{K}_{\mathbb{R}}}\right)$ temos que $i^{*}(\lambda)=-t_{0}$.

Seja $\psi \in C_{c}^{\infty}(\mathbb{R})$ com $\psi$ igual a um em uma vizinhança de $-t_{0}$. Consideremos $\tilde{\psi}(x)=$ $\psi(-x)$ então $\tilde{\psi}$ é igual a um em uma vizinhança de $t_{0}$. Temos que $\lambda\left([\psi(M) s(D)]_{\mathcal{K}_{\mathbb{R}}}\right)=$ $w\left([\psi(-M) s(-D)]_{\mathfrak{K}_{\mathbb{R}}}\right)=-w\left([\tilde{\psi}(M) s(D)]_{\mathcal{K}_{\mathbb{R}}}\right)=-\xi_{0}$ já que $s$ é uma função ímpar.

Para $t \in \mathbb{R}$ vamos definir $\left(T_{t} f\right)(x)=f(x+t), f \in L^{2}(\mathbb{R})$. É fácil verificar que $T_{t} a(M) T_{-t}=\left(T_{t} a\right)(M)$ e $T_{t} b(D) T_{-t}=b(D)$ para $a \in C_{0}(\mathbb{R})$ e $b \in C S(\mathbb{R})$. 
Dado $w \in M_{J}$ vamos definir $\tau\left([A]_{\mathcal{K}_{\mathrm{R}}}\right)=w\left(\left[T_{t} A T_{-t}\right]_{\mathcal{X}_{\mathrm{R}}}\right), A \in J_{0}$. Seja $\nu(w)=\left(t_{0}, 1\right)$. Como $\tau\left([a(M)]{\mathcal{K}_{\mathbb{R}}}\right)=w\left(\left[\left(T_{t} a\right)(M)\right]{\mathcal{K}_{\mathbb{R}}}\right)$ temos que $i^{*}(\tau)=t_{0}+t$.

Seja $\psi \in C_{c}^{\infty}(\mathbb{R}) \operatorname{com} \psi$ igual a um em uma vizinhança de $t_{0}+t$. Consideremos $\tilde{\psi}(x)=$ $\psi(x+t)$ então $\tilde{\psi}$ é igual a um em uma vizinhança de $t_{0}$. Temos que $\tau\left([\psi(M) s(D)]_{\mathcal{X}_{\mathbb{R}}}\right)=$ $w\left(\left[\left(T_{t} \psi\right)(M) s(D)\right]_{\mathcal{K}_{\mathbb{R}}}\right)=w\left([\tilde{\psi}(M) s(D)] \mathcal{K}_{\mathbb{R}}\right)=1$.

Portanto se $\left(t_{0}, 1\right) \in \operatorname{Im} \nu$ então $\left(t_{0}+t, 1\right) \in \operatorname{Im} \nu$ para todo $t \in \mathbb{R}$. Portanto usando a sobrejetividade de $i^{*}$ e o que foi visto acima temos que $\nu$ é sobrejetora.

$\triangleright$ contínua

Sejam $\left\{w_{\alpha}\right\}_{\alpha}$ uma rede em $M_{J}$ e $w \in M_{J}$ com $w_{\alpha} \rightarrow w$. Seja também $\nu(w)=(t, \xi)$ e $\nu\left(w_{\alpha}\right)=\left(t_{\alpha}, \xi_{\alpha}\right)$.

Como $w_{\alpha} \rightarrow w$ temos que $i^{*}\left(w_{\alpha}\right) \rightarrow i^{*}(w) \operatorname{logo} t_{\alpha} \rightarrow t$. Seja $\chi \in C_{c}^{\infty}(\mathbb{R})$ com $\chi$ igual a um em uma vizinhança de $t$, temos que $\xi=w\left([\chi(M) s(D)]_{\mathcal{K}_{\mathbb{R}}}\right)$. Seja $\alpha_{0}$ tal que para todo $\alpha \geq \alpha_{0}$ temos $t_{\alpha} \in U, U$ aberto de $\mathbb{R}$ onde $\chi$ é igual a um. Então para todo $\alpha \geq \alpha_{0}$ temos que $\xi_{\alpha}=w_{\alpha}\left([\chi(M) s(D)]_{\varkappa_{\mathbb{R}}}\right) \rightarrow w\left([\chi(M) s(D)]_{\mathcal{K}_{\mathbb{R}}}\right)=\xi$. Portanto $\nu$ é contínua.

$\triangleright$ a inversa é contínua

Consideremos $w, w_{\alpha},(t, \xi),\left(t_{\alpha}, \xi_{\alpha}\right)$ como antes e seja $\left(t_{\alpha}, \xi_{\alpha}\right) \rightarrow(t, \xi)$. Temos que $t_{\alpha}=i^{*}\left(w_{\alpha}\right) \rightarrow i^{*}(w)=t \log w_{\alpha}\left([a(M)]_{\mathcal{K}_{\mathbb{R}}}\right) \rightarrow w\left([a(M)]_{\mathcal{K}_{\mathbb{R}}}\right)$ para todo $a \in C_{0}(\mathbb{R})$.

Então $w_{\alpha}\left([a(M) s(D)]{\mathcal{K}_{\mathrm{R}}}_{\mathrm{R}}\right)=a\left(t_{\alpha}\right) \xi_{\alpha} \rightarrow a(t) \xi=w\left([a(M) s(D)] \mathcal{K}_{\mathrm{R}}\right)$. Como $\left\{[a(M) s(D)]_{\mathcal{K}_{\mathbb{R}}}: a \in C_{0}(\mathbb{R})\right\}$ gera $J_{0} / \mathcal{K}_{\mathbb{R}}$, segue que $w_{\alpha} \rightarrow w$. Portanto $\nu$ é um homeomorfismo.

Para concluir a demonstração basta usarmos o isomorfismo de $C_{0}(\mathbb{R} \times\{-1,1\})$ em $C_{0}(\mathbb{R} \times\{-\infty,+\infty\})$. Observar que $s( \pm \infty)= \pm 1$ e usar que os polinômios em $s$ são densos em $C S(\mathbb{R})$.

Proposição 1.16 Temos que $J_{0} \cap \mathcal{E}=\mathcal{K}_{\mathbb{R}}$.

\section{Demonstração:}

Já vimos que $\mathcal{K}_{\mathbb{R}} \subset J_{0} \cap \mathcal{E}$, vamos verificar então que $J_{0} \cap \mathcal{E} \subset \mathcal{K}_{\mathbb{R}}$. 
Decorre da Proposição 1.7 que $\mathcal{E}$ é o fecho do conjunto $\left\{\sum_{j=-N}^{N} b_{j}(M) a_{j}(D) e^{i j M}+K\right.$ : $\left.N \in \mathbb{N}, b_{j} \in C S(\mathbb{R}), a_{j} \in C_{0}(\mathbb{R}), K \in \mathcal{K}_{\mathbb{R}}\right\}$

Sabemos que $a(D-j) e^{i j M}=e^{i j M} a(D), \operatorname{logo} a_{j}(D) e^{i j M}=e^{i j M} a_{j}(D+j)$. Portanto $\mathcal{E}$ é o fecho do conjunto

$$
\left\{\sum_{j=-N}^{N} b_{j}(M) e^{i j M} a_{j}(D+j)+K: N \in \mathbb{N}, b_{j} \in C S(\mathbb{R}), a_{j} \in C_{0}(\mathbb{R}), K \in \mathcal{K}_{\mathbb{R}}\right\} .
$$

Seja $A \in\left(J_{0} \cap \mathcal{E}\right)$ e consideremos $\chi_{j} \in C_{c}^{\infty}(\mathbb{R})$ com $0 \leq \chi_{j} \leq 1$ e $\chi_{j} \equiv 1$ em $(-j, j)$ com $j \in \mathbb{N}$. Como $A \in J_{0}$ podemos calcular o $\sigma$ - símbolo de $A$, $\operatorname{logo} \sigma_{A}=\lim _{j \rightarrow \infty} \sigma_{\chi_{j} A}$. Portanto $[A]_{\mathcal{K}_{\mathbb{R}}}=\lim _{j \rightarrow \infty}\left[\chi_{j} A\right]_{\mathcal{K}_{\mathbb{R}}}$ em $J_{0} / \mathcal{K}_{\mathbb{R}}$, ou seja $\left\|\left[\chi_{j} A\right]_{\mathcal{K}_{\mathbb{R}}}-[A]_{\mathcal{K}_{\mathbb{R}}}\right\| \longrightarrow 0$ quando $j \longrightarrow \infty$. Logo existem $\left(K_{j}\right)_{j}$ onde $K_{j} \in \mathcal{K}_{\mathbb{R}}$ tais que $\lim _{j \rightarrow \infty} \chi_{j} A+K_{j}=A$.

Como $A \in \mathcal{E}$ temos, também, que $A=\lim _{k \rightarrow \infty} \sum_{l=-N_{k}}^{N_{k}} b_{l}^{k}(M) e^{i l M} a_{l}^{k}(D+l)+S_{k}$ onde $b_{l}^{k} \in C S(\mathbb{R}), a_{l}^{k} \in C_{0}(\mathbb{R})$ e $S_{k} \in \mathcal{K}_{\mathbb{R}}$.

Logo

$$
A=\lim _{j \rightarrow \infty} \lim _{k \rightarrow \infty} \sum_{l=-N_{k}}^{N_{k}} \chi_{j}(M) b_{l}^{k}(M) e^{i l M} a_{l}^{k}(D+l)+S_{k}+K_{j} .
$$

Como $\chi_{j}(M) b_{l}^{k}(M) e^{i l M} a_{l}^{k}(D+l)$ é compacto, vem que $A$ é o limite de uma soma de compactos, logo compacto. Portanto $\left(J_{0} \cap \mathcal{E}\right) \subset \mathcal{K}_{\mathbb{R}}$, assim $J_{0} \cap \mathcal{E}=\mathcal{K}_{\mathbb{R}}$.

\section{$1.4 \mathrm{O}$ espaço símbolo de $\mathcal{A}$}

Nesta seção calcularemos o espaço símbolo, $M_{A}$, de $\mathcal{A}$ e daremos uma fórmula explicíta para o homomorfismo $\varphi: \mathcal{A} \longrightarrow C\left(M_{A}\right)$.

Teorema 1.17 $O$ espaço símbolo $M_{A} d a C^{*}$-álgebra $\mathcal{A}$ é dado como:

$$
M_{A}=M_{\sharp} \times\{-\infty,+\infty\} .
$$

O б-símbolo é dado por:

$$
\begin{gathered}
\sigma_{a}(m, \pm \infty)=a(m), m \in M_{\sharp}, a \in \mathcal{A}^{\sharp} \quad e \\
\sigma_{b(D)}(m, \pm \infty)=b( \pm \infty), m \in M_{\sharp}, b \in C S(\mathbb{R}) .
\end{gathered}
$$




\section{Demonstração:}

Consideremos as aplicações

$$
\begin{array}{cccc}
i_{1}: & \mathcal{A}^{\sharp} & \longrightarrow \mathcal{A} / \mathcal{E} \\
a(M) & \longmapsto[a(M)]_{\mathcal{E}}
\end{array}
$$

e

$$
\begin{aligned}
i_{2}: C S(\mathbb{R}) & \longrightarrow \mathcal{A} / \mathcal{E} \\
b & \longmapsto[b(D)]_{\mathcal{E}} .
\end{aligned}
$$

E suas aplicações duais

$$
\begin{aligned}
i_{1}^{*}: M_{A} & \longrightarrow M_{\sharp} \\
\mathrm{e} & \\
i_{2}^{*}: M_{A} & \longrightarrow[-\infty,+\infty],
\end{aligned}
$$

onde dado $w \in M_{A}$ temos $i_{j}^{*}(w)=w \circ i_{j}, j=1$ e 2 .

Consideremos $i$ o produto das duais:

$$
i: M_{A} \longrightarrow M_{\sharp} \times[-\infty,+\infty],
$$

com $w \in M_{A}$ tendo imagem $i(w)=\left(w \circ i_{1}, w \circ i_{2}\right)$.

Como $\operatorname{Im} i_{1}$ e $\operatorname{Im} i_{2}$ geram $\mathcal{A} / \mathcal{E}$ é fácil ver que $i$ é injetora. Como $M_{A}$ é compacto (Teorema 1.3.5, [28]), $M_{\sharp} \times[-\infty,+\infty]$ é Hausdorff e $i$ é contínua temos que $i$ é um homeomorfismo sobre a imagem.

Usando este homeomorfismo como identificação temos que:

$$
\sigma_{a}(m, t)=\hat{a}(m) \quad \text { e } \quad \sigma_{b(D)}(m, t)=b(t)
$$

para $a \in \mathcal{A}^{\sharp}, b \in C S(\mathbb{R})$ e $(m, t) \in M_{\sharp} \times[-\infty,+\infty]$.

Até aqui temos que $M_{A} \subset M_{\sharp} \times[-\infty,+\infty]$. Mas demonstraremos nos Lemas 1.20, 1.21 e 1.22 respectivamente que $M_{A} \cap(\mathbb{R} \times\{-\infty,+\infty\}) \neq \emptyset ;(x,+\infty) \in M_{A} \cap(\mathbb{R} \times$ $\{-\infty,+\infty\})$ se, e só se, $(-x,-\infty) \in M_{A} \cap(\mathbb{R} \times\{-\infty,+\infty\}) ;(x,+\infty) \in M_{A} \cap(\mathbb{R} \times$ $\{-\infty,+\infty\})$ se, e só se, $(y,+\infty) \in M_{A} \cap(\mathbb{R} \times\{-\infty,+\infty\})$, para todo $y \in \mathbb{R}$. Logo $\mathbb{R} \times\{-\infty,+\infty\} \subset M_{A}$ e como $\mathbb{R}$ é denso em $M_{\sharp}$ temos que $M_{\sharp} \times\{-\infty,+\infty\} \subset M_{A}$. No Lema 1.19 demonstraremos que $(m, t) \notin M_{A}$ se $|t|<\infty, \log 0 M_{A}=M_{\sharp} \times\{-\infty,+\infty\}$. 
Lema 1.18 Dado qualquer $x \in M_{\sharp}$ existe $y \in[-\infty,+\infty]$ tal que $(x, y) \in M_{A}$.

\section{Demonstração:}

Dados $a(M) \in \mathcal{E} \cap \mathcal{A}^{\sharp}$ e $\chi \in C_{c}^{\infty}(\mathbb{R})$ temos que $\chi(M) a(M) \in\left(J_{0} \cap \mathcal{E}\right)=\mathcal{K}_{\mathbb{R}}$, já que $b(M) \in J_{0}$ se $b \in C_{0}(\mathbb{R})$ e $\mathcal{E}$ é ideal de $\mathcal{A}$. Temos então que $\chi a(M)=0$, logo $\chi a \equiv 0$ para todo $\chi \in C_{c}^{\infty}(\mathbb{R})$ o que implica que $a \equiv 0$. Portanto $\mathcal{E} \cap \mathcal{A}^{\sharp}=\{0\}$.

Consideremos a aplicação $i_{1}$ do teorema acima, como $\mathcal{E} \cap \mathcal{A}^{\sharp}=\{0\}$ temos que $i_{1}$ é um isomorfismo sobre a imagem, logo $i_{1}^{*}$ é sobrejetora (veja, por exemplo, pág. 314 de [10]).

Então dado $x \in M_{\sharp}$ existe $x^{\prime} \in M_{A}$ tal que $i_{1}^{*}\left(x^{\prime}\right)=x$ e $i\left(x^{\prime}\right)=\left(i_{1}^{*}\left(x^{\prime}\right), i_{2}^{*}\left(x^{\prime}\right)\right)=$ $\left(x, i_{2}^{*}\left(x^{\prime}\right)\right) \in M_{A}$.

Lema $1.19(m, t) \notin M_{A}$ se $|t|<\infty$.

\section{Demonstração:}

Dado $t_{0} \in \mathbb{R}$, podemos escolher $a \in C_{0}(\mathbb{R})$ tal que $a\left(t_{0}\right) \neq 0$. Temos que $a(D) \in \mathcal{E}=$ $\operatorname{Ker} \sigma, \log \sigma_{a(D)}(m, t)=a(t)=0$, para todo $(m, t) \in M_{A}$. Assim $\left(m, t_{0}\right) \notin M_{A}$, para qualquer $m \in M_{\sharp}$ já que $a\left(t_{0}\right) \neq 0$.

Lema 1.20 $M_{A} \cap(\mathbb{R} \times\{-\infty,+\infty\}) \neq \emptyset$.

\section{Demonstração:}

Dado $x \in \mathbb{R} \subset M_{\sharp}$ temos pelo Lema 1.18 que existe $y \in[-\infty,+\infty]$ tal que $(x, y) \in$ $M_{A}$. E pelo Lema 1.19 temos que se $|y|<\infty$ então $(x, y) \notin M_{A}$. Portanto $(x, y) \in$ $M_{A} \cap \mathbb{R} \times\{-\infty,+\infty\}$.

Lema $1.21(x,+\infty) \in M_{A} \cap(\mathbb{R} \times\{-\infty,+\infty\}) \Longleftrightarrow(-x,-\infty) \in M_{A} \cap(\mathbb{R} \times\{-\infty,+\infty\})$.

\section{Demonstração:}

Consideremos o operador paridade $P$, isto é, $(P f)(x)=f(-x), f \in L^{2}(\mathbb{R})$. É fácil verificar que $P a(M) P=a(-M)$ e $P b(D) P=b(-D)$ para $a \in \mathcal{A}^{\sharp}$ e $b \in C S(\mathbb{R})$.

Seja $g: \mathcal{A} / \mathcal{E} \longrightarrow \mathcal{A} / \mathcal{E}$, onde $[a]_{\mathcal{E}} \mapsto[P a P]_{\mathcal{E}}$. Dado $\lambda \in M_{A}$ definimos $w=\lambda \circ g$. Pode-se verificar sem dificuldades que $w \in M_{A}$. 
Se $i(\lambda)=(x,+\infty)$ então $i(w)=(-x,-\infty)$ e se $i(\lambda)=(-x,-\infty)$ então $i(w)=$ $(x,+\infty)$. Portanto temos o resultado.

Lema $1.22(x,+\infty) \in M_{A} \cap(\mathbb{R} \times\{-\infty,+\infty\}) \Longleftrightarrow(y,+\infty) \in M_{A} \cap(\mathbb{R} \times\{-\infty,+\infty\})$, para todo $y \in \mathbb{R}$.

\section{Demonstração:}

Dado $t \in \mathbb{R}$ vamos definir $\left(T_{t} f\right)(x)=f(x+t), f \in L^{2}(\mathbb{R})$. É fácil verificar que $T_{t} a(M) T_{-t}=\left(T_{t} a\right)(M)$ e $T_{t} b(D) T_{-t}=b(D)$ para $a \in \mathcal{A}^{\sharp}$ e $b \in C S(\mathbb{R})$.

Seja $g: \mathcal{A} / \mathcal{E} \longrightarrow \mathcal{A} / \mathcal{E}$, onde $[a]_{\mathcal{E}} \mapsto\left[T_{t} a T_{-t}\right]_{\mathcal{E}}$, a partir daqui a demonstração é análoga a do Lema anterior.

\section{$1.5 \mathrm{O}$ quociente $\varepsilon / \mathcal{K}_{\mathbb{R}}$}

Nesta seção vamos construir o isomorfismo de $\mathcal{E} / \mathcal{K}_{\mathbb{R}}$ para $C\left(S^{1} \times\{-1,1\}, \mathcal{K}_{\mathbb{Z}}\right)$.

Seja $S L$ a $C^{*}$ - subálgebra de $\mathcal{L}\left(L^{2}\left(S^{1}\right)\right)$ gerada pelos operadores $a(M)$ de multiplicação por funções $a \in C^{\infty}\left(S^{1}\right)$, e por todos os operadores $b\left(D_{\theta}\right):=F_{d}^{-1} b(M) F_{d}$, onde

$$
\begin{aligned}
F_{d}: \quad L^{2}\left(S^{1}\right) & \rightarrow L^{2}(\mathbb{Z}) \\
f & \mapsto\left(f_{j}\right)_{j}
\end{aligned}
$$

é a transformada de Fourier discreta, com $f_{j}=\frac{1}{\sqrt{2 \pi}} \int_{0}^{2 \pi} e^{-i j \theta} f\left(e^{i \theta}\right) d \theta, b \in C S(\mathbb{Z}), b(M)\left(u_{j}\right)_{j}$ $=\left(b(j) u_{j}\right)_{j}$ e $C S(\mathbb{Z})$ é o conjunto das sequências $\left(b_{j}\right)_{j}$ que possuem limites quando $j$ tende $\mathrm{a}+\infty$ e $-\infty$.

Podemos verificar que $S L$ é irredutível (verificação análoga à de $\varepsilon$ ) e que todos os seus comutadores são compactos (veja, por exemplo, [10]), então pelo Teorema 1.2 temos que $\mathcal{K}_{S^{1}} \subset S L$. Usando a mesma estratégia do Teorema 1.17 podemos verificar que o espaço símbolo de $S L$ é $M_{S L}=S^{1} \times\{-1,1\}$. Logo pelo Teorema de Gelfand temos que $S L / \mathcal{K}_{S^{1}}$ é isomorfo a $C\left(S^{1} \times\{-1,1\}\right)$ e que $\sigma_{a}(z, \pm 1)=a(z)$ e $\sigma_{b\left(D_{\theta}\right)}(z, \pm 1)=b( \pm \infty)$, $a \in C^{\infty}\left(S^{1}\right)$ e $b \in C S(\mathbb{Z})$. 
Logo $A \otimes K \longmapsto \sigma_{A} \otimes K$ induz um *-isomorfismo

$$
\frac{S L \hat{\otimes} \mathcal{K}_{\mathbb{Z}}}{\mathcal{K}_{S^{1} \times \mathbb{Z}}} \cong \frac{S L \hat{\otimes} \mathcal{K}_{\mathbb{Z}}}{\mathcal{K}_{S^{1}} \hat{\otimes} \mathcal{K}_{\mathbb{Z}}} \cong \frac{S L}{\mathcal{K}_{S^{1}}} \hat{\otimes} \mathcal{K}_{\mathbb{Z}} \cong C\left(M_{S L}\right) \hat{\otimes} \mathcal{K}_{\mathbb{Z}} \cong C\left(M_{S L}, \mathcal{K}_{\mathbb{Z}}\right)
$$

(veja [11], para mais detalhes). Acima $\hat{\otimes}$ denota o produto tensorial de $C^{*}$-álgebras nucleares. Em particular $S L \hat{\otimes} \mathcal{K}_{\mathbb{Z}}$ denota o fecho do produto tensorial algébrico de $S L \otimes \mathcal{K}_{\mathbb{Z}}$ em $\mathcal{L}\left(L^{2}\left(S^{1}\right) \hat{\otimes} L^{2}(\mathbb{Z})\right)$.

Dado $u \in L^{2}(\mathbb{R})$ denotamos:

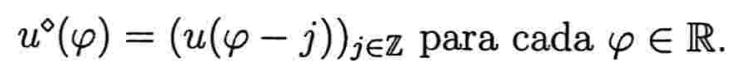

Temos, pelo Teorema de Fubini, que $u^{\diamond}(\varphi) \in L^{2}(\mathbb{Z})$ para quase todo $\varphi \in \mathbb{R}$.

Para cada $\varphi \in \mathbb{R}$ definimos $Y_{\varphi}=F_{d} M^{-\varphi} F_{d}^{-1}$, onde $M^{-\varphi} \in \mathcal{L}\left(L^{2}\left(S^{1}\right)\right)$ é o operador de multiplicação pela função $\left(z \mapsto z^{-\varphi}\right)$, isto é $\left(M^{-\varphi} f\right)\left(e^{i \theta}\right)=e^{-i \varphi \theta} f\left(e^{i \theta}\right)$. Podemos verificar que $Y_{\varphi}, \varphi \in \mathbb{R}$, é uma família de operadores unitários de $\mathcal{L}_{\mathbb{Z}}$ com as seguintes propriedades:

$$
Y_{\varphi} Y_{w}=Y_{\varphi+w}, \varphi, w \in \mathbb{R} \quad \text { e } \quad\left(Y_{k} u\right)_{j}=u_{j+k}, k \in \mathbb{Z}, u \in L^{2}(\mathbb{Z}) .
$$

Podemos então definir o operador unitário (com $S^{1}=\left\{e^{2 \pi i \varphi}: \varphi \in \mathbb{R}\right\}$ )

$$
\begin{aligned}
W: L^{2}(\mathbb{R}) & \longrightarrow L^{2}\left(S^{1}, d \varphi ; L^{2}(\mathbb{Z})\right) \\
u & \longmapsto(W u)(\varphi)=Y_{\varphi} u^{\diamond}(\varphi) .
\end{aligned}
$$

Usando a primeira propriedade de $Y_{\varphi}$ verifica-se facilmente que se $b \in C S(\mathbb{R})$, então $\varphi \mapsto Y_{\varphi} b(\varphi-M) Y_{-\varphi}$ é 1-periódica e pode ser vista, portanto como um elemento de $C\left(S^{1}, \mathcal{L}_{\mathbb{Z}}\right)$.

Então pelo Teorema 2.6 de [13], que enunciamos abaixo, podemos definir o isomorfismo $\psi$.

Teorema 1.23 Temos que $W \hat{\varepsilon} W^{-1}=S L \hat{\otimes} \mathcal{K}_{\mathbb{Z}}$.

Além disso, para $b \in C S(\mathbb{R}), a \in C_{0}(\mathbb{R})$ e $j \in \mathbb{Z}$, temos:

$$
\begin{gathered}
Y_{\varphi} a(\varphi-M) Y_{-\varphi} \in C\left(S^{1}, \mathcal{K}_{\mathbb{Z}}\right) e \\
W\left(b(D) a T_{j}\right) W^{-1}=b\left(D_{\theta}\right) Y_{\varphi} a(\varphi-M) Y_{-\varphi-j}+K, \quad K \in \mathcal{K}_{S^{1} \times \mathbb{Z}}
\end{gathered}
$$

onde denotamos por $f(\varphi)$ funções $S^{1} \ni e^{2 \pi i \varphi} \mapsto f(\varphi) \in \mathcal{K}_{\mathbb{Z}}$. 


\section{Proposição 1.24 Existe um *-isomorfismo}

$$
\psi: \mathcal{E} / \mathcal{K}_{\mathbb{R}} \longrightarrow C\left(M_{S L}, \mathcal{K}_{\mathbb{Z}}\right)
$$

tal que se $\tilde{\gamma}$ denota a composição de $\psi$ com a projeção canônica $\mathcal{E} \longrightarrow \mathcal{E} / \mathcal{K}_{\mathbb{R}}$, e se $E \in \mathcal{E}$ satisfaz $F^{-1} E F=b(D) a T_{j}, b \in C S(\mathbb{R}), a \in C_{0}(\mathbb{R}), j \in \mathbb{Z}$ então temos:

$$
\tilde{\gamma}_{E}(\varphi, \pm 1)=b( \pm \infty) Y_{\varphi} a(\varphi-M) Y_{-\varphi-j}, \quad\left(e^{2 \pi i \varphi}, \pm 1\right) \in M_{S L} .
$$

\section{Demonstração:}

Vamos definir $\psi$ como sendo a composição das aplicações:

$$
\mathcal{E} / \mathcal{K}_{\mathbb{R}} \longrightarrow \hat{\mathcal{E}} / \mathcal{K}_{\mathbb{R}} \longrightarrow \frac{S L \hat{\otimes} \mathcal{K}_{\mathbb{Z}}}{\mathcal{K}_{S^{1} \times \mathbb{Z}}} \longrightarrow C\left(M_{S L}, \mathcal{K}_{\mathbb{Z}}\right)
$$

onde, a primeira aplicação leva $A+\mathcal{K}_{\mathbb{R}}$ para $F^{-1} A F+\mathcal{K}_{\mathbb{R}}$, a segunda para $W F^{-1} A F W^{-1}+$ $\mathcal{K}_{S^{1} \times \mathbb{Z}}$ e a última é o isomorfismo (1.6). Pelo Teorema 1.23 e pela equação (1.7), temos o resultado para $\tilde{\gamma}_{E}(\varphi, \pm 1)$.

Estenderemos $\tilde{\gamma}$, definida sobre $\mathcal{E}$, para a álgebra inteira $\mathcal{A}$. Como $\mathcal{E} / \mathcal{K}_{\mathbb{R}}$ é um ideal de $\mathcal{A} / \mathcal{K}_{\mathbb{R}}$, todo $A \in \mathcal{A}$ define um operador $T_{A}$ em $\mathcal{L}\left(\mathcal{E} / \mathcal{K}_{\mathbb{R}}\right)$ por

$$
T_{A}\left(E+\mathcal{K}_{\mathbb{R}}\right)=A E+\mathcal{K}_{\mathbb{R}}
$$

Assim define-se:

$$
T: \mathcal{A} \longrightarrow \mathcal{L}\left(\mathcal{E} / \mathcal{K}_{\mathbb{R}}\right)
$$

Está claro que $\left\|T_{A}\right\| \leq\|A\|$. Vamos definir

$$
\begin{aligned}
\gamma: \mathcal{A} & \longrightarrow \mathcal{L}\left(C\left(M_{S L}, \mathcal{K}_{\mathbb{Z}}\right)\right) \\
A & \longmapsto \psi T_{A} \psi^{-1} .
\end{aligned}
$$

Para $E \in \mathcal{E}, \gamma_{E}$ é uma multiplicação por $\tilde{\gamma}_{E} \in C\left(M_{S L}, \mathcal{K}_{\mathbb{Z}}\right)$ da Proposição 1.24 . Identificando funções de $C\left(M_{S L}, \mathcal{K}_{\mathbb{Z}}\right)$ com operadores de multiplicação correspondentes em $\mathcal{L}\left(C\left(M_{S L}, \mathcal{K}_{\mathbb{Z}}\right)\right)$, nós podemos então dizer que $\gamma$ estende $\tilde{\gamma}$.

Proposição 1.25 Existe um *-homomorfismo

$$
\gamma: \mathcal{A} \longrightarrow C\left(M_{S L}, \mathcal{L}_{\mathbb{Z}}\right)
$$


estendendo $\tilde{\gamma}$ da Proposição 1.24. Sobre os geradores de $\mathcal{A}, \gamma$ é dada por: .

$$
\begin{gathered}
\gamma_{a}(\varphi, \pm 1)=a( \pm \infty), a \in C S(\mathbb{R}), \\
\gamma_{b(D)}(\varphi, \pm 1)=Y_{\varphi} b(M-\varphi) Y_{-\varphi}, b \in C S(\mathbb{R}) e \\
\gamma_{e^{i j M}}(\varphi, \pm 1)=Y_{-j}, j \in \mathbb{Z} .
\end{gathered}
$$

\section{Demonstração:}

É suficiente provar as equações acima para a aplicação $\gamma$ definida em (1.8). Por continuidade, a imagem de $\gamma$ estará então contida em $C\left(M_{S L}, \mathcal{L}_{\mathbb{Z}}\right)$ considerada como uma subálgebra fechada de $\mathcal{L}\left(C\left(M_{S L}, \mathcal{K}_{\mathbb{Z}}\right)\right)$.

Dado $a \in C S(\mathbb{R})$, precisamos calcular $\tilde{\gamma}_{a E}$ em termos de $\tilde{\gamma}_{E}$, para $E \in \mathcal{E}$. Pela Proposição 1.7, temos que basta considerar $E$ tal que

$$
F^{-1} E F=d(D) c T_{k}, d \in C S(\mathbb{R}), c \in C_{0}(\mathbb{R}), \text { e } k \in \mathbb{Z} .
$$

Obtemos então $F^{-1}(a E) F=(a d)(D) c T_{j}$ e portanto, pela Proposição 1.24,

$$
\tilde{\gamma}_{a E}(\varphi, \pm 1)=a( \pm \infty) d( \pm \infty) Y_{\varphi} c(\varphi-M) Y_{-\varphi-j}=a( \pm \infty) \tilde{\gamma}_{E}(\varphi, \pm 1)
$$

Para $E$ como acima e $b \in C S(\mathbb{R})$,

$$
F^{-1}(b(D) E) F=b(-M) d(D) c T_{j}=d(D) b(-M) c T_{j}+K, K \in \mathcal{K}_{\mathbb{R}},
$$

e, assim,

$$
\tilde{\gamma}_{b(D) E}(\varphi, \pm 1)=d( \pm \infty) Y_{\varphi} b(-\varphi+M) c(\varphi-M) Y_{-\varphi-j}=Y_{\varphi} b(M-\varphi) Y_{-\varphi} \tilde{\gamma}_{E}(\varphi, \pm 1) .
$$

Para o mesmo $E$ temos

$$
F^{-1} e^{i j M} E F=T_{j} d(D) c T_{k}=d(D) c(M+j) T_{j+k}
$$

e, então, usando que $Y_{-j} c(\varphi-M)=c(\varphi-M+j) Y_{-j}$,

$$
\tilde{\gamma}_{e^{i j M} E}(\varphi, \pm 1)=d( \pm \infty) Y_{\varphi} c(\varphi-M+j) Y_{-\varphi-j-k}=Y_{-j} d( \pm \infty) Y_{\varphi} c(\varphi-M) Y_{-\varphi-k}=
$$

$Y_{-j} \tilde{\gamma}_{E}(\varphi, \pm 1)$. A aplicação preserva $*$, pois preserva $*$ nos geradores.

Proposição 1.26 Seja $\mathcal{A}^{\circ} \subset \mathcal{A}$ a subálgebra fechada gerada pelos operadores do tipo 
ii) e iii) da página 17. Se $A \in \mathcal{A}^{\diamond}$ então $f=\left(W F^{-1}\right) A\left(W F^{-1}\right)^{-1} \in C\left(S^{1}, \mathcal{L}_{\mathbb{Z}}\right)$ e $\gamma_{A}(z, 1)=f(z)=\gamma_{A}(z,-1)$ para todo $z \in S^{1}$. Em particular, $\left\|\gamma_{A}\right\|=\|A\|$ para todo $A \in \mathcal{A}^{\circ}$.

\section{Demonstração:}

Dado $b \in C S(\mathbb{R})$, para cada $\varphi \in \mathbb{R},(b(\varphi-j))_{j \in \mathbb{Z}} \in C S(\mathbb{Z})$; denotamos por $b(\varphi-M) \in$ $\mathcal{L}_{\mathbb{Z}}$ o operador de multiplicação por esta sequência.

Para $j \in \mathbb{Z}, \varphi \in \mathbb{R}$ e $u \in L^{2}(\mathbb{R})$ temos,

$$
\left(Y_{\varphi} b(\varphi-M) Y_{-j} Y_{-\varphi}\right)\left(Y_{\varphi} u^{\circ}(\varphi)\right)=Y_{\varphi}(b(\varphi-k) u(\varphi-k+j))_{k \in \mathbb{Z}},
$$

$\log 0 W b T_{j} W^{-1}=Y_{\varphi} b(\varphi-M) Y_{-\varphi-j}$, para $b \in C S(\mathbb{R})$ e $j \in \mathbb{Z}$.

Por outro lado segue das equações da Proposição 1.25 que

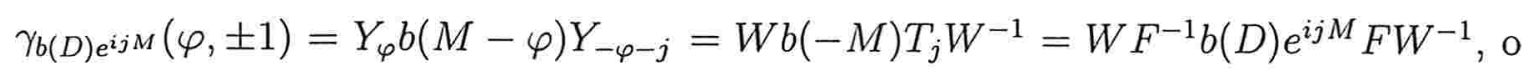
que prova que $\gamma_{A}(z, 1)=\gamma_{A}(z,-1)$, para todo $A \in \mathcal{A}^{\circ}$. Como $W F^{-1}$ é um operador unitário segue que $\left\|\gamma_{A}\right\|=\|A\|$ para todo $A \in \mathcal{A}^{\circ}$.

Proposição 1.27 Seja $J_{0}$ a $C^{*}$ - álgebra definida na seção 1.3. Temos que Ker $\gamma=J_{0}$.

\section{Demonstração:}

Se $A \in J_{0}$, vimos na demonstração da Proposição 1.16 que $\lim _{j \rightarrow \infty} \chi_{j} A+K_{j}=A$ onde $\chi_{j} \in C_{c}^{\infty}(\mathbb{R}) \operatorname{com} 0 \leq \chi_{j} \leq 1$ e $\chi_{j} \equiv 1$ em $(-j, j) \operatorname{com} j \in \mathbb{N} ; K_{j} \in \mathcal{K}_{\mathbb{R}}$.

Agora se $A \in \mathcal{A}$ e $\lim _{j \rightarrow \infty} \chi_{j} A+K_{j}=A$ temos que $A \in J_{0}$, já que $A=\lim _{k \rightarrow \infty} \sum_{l=-N_{k}}^{N_{k}} b_{l}^{k}(M) a_{l}^{k}(D) e^{i l M}+C ; N_{k} \in \mathbb{N}, b_{l} \in C S(\mathbb{R}), a_{l} \in C S(\mathbb{R}), C \in \mathcal{K}_{\mathbb{R}} \mathrm{e}$ $a(D-j) e^{i j M}=e^{i j M} a(D)$.

Com isto provamos que $A \in \mathcal{A}$ pertence a $J_{0}$ se, e só se, $\lim _{j \rightarrow \infty} \chi_{j} A+K_{j}=A, K_{j} \in \mathcal{K}_{\mathbb{R}}$.

Podemos então concluir que $A \in \mathcal{A}$ pertence a $J_{0}$ se, e só se, $\sigma_{A}(m, \pm \infty)=0$ para todo $m \in S_{+}^{1} \cup S_{-}^{1}$, onde $S_{+}^{1}=S^{1} \times\{+\infty\}$ e $S_{-}^{1}=S^{1} \times\{-\infty\}$. Pela Proposição $3.4 \mathrm{de}$ [13] temos que

$$
\sup \left\{\left|\sigma_{A}(m, \xi)\right|: x \in S_{+}^{1} \cup S_{-}^{1}, \xi= \pm \infty\right\} \leq\left\|\gamma_{A}\right\| .
$$


Logo se $A \in \operatorname{Ker} \gamma$ então $\sigma_{A}(m, \pm \infty)=0$ para $m \in S_{+}^{1} \cup S_{-}^{1}$. E vimos acima que isto ocorre se, e só se, $A \in J_{0}$, portanto $\operatorname{Ker} \gamma=J_{0}$. 


\section{Capítulo 2}

\section{A álgebra $C$}

Neste capítulo vamos estudar a $C^{*}$ - subálgebra de $\mathcal{L}\left(L^{2}(\mathbb{R})\right)$, que chamamos $\mathcal{C}$, gerada por operadores de multiplicação $a(M)$ e $b(D):=F^{-1} b(M) F \operatorname{com} a, b \in C S(\mathbb{R})$.

Calcularemos sua K - teoria e obteremos uma fórmula para o índice de Fredholm.

Definição 2.1 Seja e a $C^{*}$ - subálgebra de $\mathcal{L}\left(L^{2}(\mathbb{R})\right)$ obtida como o fecho da $C^{*}$ - álgebra gerada por:

i) operadores de multiplicação a $(M), a \in C S(\mathbb{R})$.

ii) operadores da forma $b(D):=F^{-1} b(M) F, b \in C S(\mathbb{R})$.

É óbvio que $\mathcal{C}$ é uma $C^{*}$ - subálgebra de $\mathcal{A}$, introduzida no Capítulo 1.

\subsection{O espaço símbolo de $\mathcal{C}$}

Nesta seção calcularemos o espaço símbolo, $M_{C}$, de $\mathcal{C}$ e daremos uma fórmula explícita para o homomorfismo $\varphi: \mathrm{e} \longrightarrow C\left(M_{C}\right)$.

Proposição 2.2 A $C^{*}$-álgebra C é irredutivel.

Demonstração: 
Temos que $\{a u: a \in \mathcal{C}\}$ é denso em $L^{2}(\mathbb{R})$, para todo $u$ não nulo. Pois do Lema 1.13 temos que $\left\{a u: a \in J_{0}\right\}$ é denso em $L^{2}(\mathbb{R})$, para todo $u$ não nulo e $J_{0} \subset \mathcal{C}$. Então pelo Lema 1.2 temos que $\mathcal{C}$ é irredutível

Usando o Teorema 1.3, (1.1) e a Observação $1.61 / 2$ temos que

$$
\mathcal{K}_{\mathbb{R}} \subset \mathcal{C}
$$

Seja $J$ o ideal comutador de $\mathcal{C}$, temos

$$
J \subset \mathcal{K}_{\mathbb{R}}
$$

já que $[a(M), b(D)] \in \mathcal{K}_{\mathbb{R}}, a, b \in C S(\mathbb{R})$. Por (2.1), (2.2) e pela Observação $1.61 / 2$ temos $J=\mathcal{K}_{\mathbb{R}}$.

Teorema 2.3 O espaço símbolo $M_{C}$ da $C^{*}$ - álgebra $\mathrm{e}$ é dado como

$$
M_{C}=\{(x, \xi) \in[-\infty,+\infty] \times[-\infty,+\infty]:|x|+|\xi|=\infty\} .
$$

O $\sigma$ - símbolo é dado por:

$$
\begin{gathered}
\sigma_{a}(x, \xi)=a(x),(x, \xi) \in M_{C}, \\
\sigma_{b(D)}(x, \xi)=b(\xi),(x, \xi) \in M_{C} .
\end{gathered}
$$

\section{Demonstração:}

Consideremos as aplicações

$$
\begin{aligned}
i_{1}: C S(\mathbb{R}) & \longrightarrow \mathrm{C} / \mathcal{K}_{\mathbb{R}} \\
a & \longmapsto[a(M)]_{\mathcal{K}_{\mathbb{R}}} \\
& \mathrm{e} \\
i_{2}: C S(\mathbb{R}) & \longrightarrow \mathrm{C} / \mathcal{K}_{\mathbb{R}} \\
b & \longmapsto[b(D)]_{\mathcal{K}_{\mathbb{R}}}
\end{aligned}
$$

Note que $i_{j}, j=1,2$, é injetora já que $a(M) \in \mathcal{K}_{\mathbb{R}}$ se, e só se, $a \equiv 0$.

Temos que o espectro de $C S(\mathbb{R})$ é o conjunto $[-\infty,+\infty]$. Consideremos as aplicaçōes duais de $i_{1}$ e $i_{2}$ :

$$
\begin{gathered}
i_{1}^{*}: M_{C} \longrightarrow[-\infty,+\infty] \quad \mathrm{e} \\
i_{2}^{*}: M_{C} \longrightarrow[-\infty,+\infty],
\end{gathered}
$$


onde dado $w \in M_{C}$ temos $i_{j}^{*}(w)=w \circ i_{j}, j=1,2$. Como $i_{j}, j=1,2$ é injetora temos que $i_{j}^{*}, j=1,2$ é sobrejetora (veja, por exemplo, [3], pág. 314).

Seja $i$ o produto das duais

$$
i: M_{C} \longrightarrow[-\infty,+\infty] \times[-\infty,+\infty]
$$

onde $w \in M_{C}$ tem imagem $i(w)=\left(w \circ i_{1}, w \circ i_{2}\right)$.

Como $\operatorname{Im} i_{1}$ e $\operatorname{Im} i_{2}$ geram $\mathcal{C} / \mathcal{K}_{\mathbb{R}}$ é fácil ver que $i$ é injetora. Como $M_{C}$ é compacto, $[-\infty,+\infty] \times[-\infty,+\infty]$ é Hausdorff e $i$ é contínua temos que $i$ é um homeomorfismo sobre a imagem.

Usando este homeomorfismo como identificação temos

$$
\sigma_{a}(x, \xi)=a(x) \quad \text { e } \quad \sigma_{b(D)}(x, \xi)=b(\xi) .
$$

Sabemos que $A=a(M) b(D) \in \mathcal{K}_{\mathbb{R}}=\operatorname{Ker} \sigma$ para $a, b \in C_{0}(\mathbb{R})$ e podemos escolher $a$ e $b$ que nunca se anulam em $\mathbb{R}$. Como

$$
\sigma_{A}(x, \xi)=a(x) b(\xi)=0 \quad \forall \quad(x, \xi) \in M_{C},
$$

e $a(x) b(\xi)=0$ se, e somente se, $|x|=\infty$ ou $|\xi|=\infty$ ou ambos, segue que $M_{C} \subset\{(x, \xi) \in$ $[-\infty,+\infty] \times[-\infty,+\infty]:|x|+|\xi|=\infty\}$

Seja $w=(x, \xi) \in M_{C}$ e vamos definir $\lambda\left([A]_{\mathcal{K}_{\mathrm{R}}}\right)=w\left(\left[F^{-1} A F\right]_{\mathcal{K}_{\mathrm{R}}}\right)$. Temos que $\lambda\left([a(M)]_{\mathcal{K}_{\mathbb{R}}}\right)=w\left([a(D)]_{\mathcal{K}_{\mathbb{R}}}\right)=a(\xi)$ e $\lambda\left([b(D)]_{\mathcal{K}_{\mathbb{R}}}\right)=w\left([b(-M)]_{\mathcal{K}_{\mathbb{R}}}\right)=b(-x)$, logo $\lambda=$ $(\xi, x)$.

Usando o operador translação do Lema 1.22 temos que dado $x \in \mathbb{R},(x,+\infty) \in M_{C}$ se, e só se, $(y,+\infty) \in M_{C}$ para todo $y \in \mathbb{R}$. E usando o operador paridade do Lema 1.21 temos que dado $x \in \mathbb{R},(x,+\infty) \in M_{C}$ se, e só se, $(-x,-\infty) \in M_{C}$. Usando a sobrejetividade das aplicações duais, temos $M_{C}=\{(x, \xi) \in[-\infty,+\infty] \times[-\infty,+\infty]$ : $|x|+|\xi|=\infty\}$.

\subsection{Espaços de Hardy e operadores de Toeplitz}

Nesta seção apresentaremos a definição de espaços de Hardy no círculo, $H^{2}\left(S^{1}\right)$, e na reta, $H^{2}(\mathbb{R})$, e provaremos que $U\left(H^{2}\left(S^{1}\right)\right)=H^{2}(\mathbb{R})\left(\operatorname{com} S^{1}=\mathbb{R} / 2 \pi \mathbb{Z}\right)$, onde $U$ é um 
operador unitário. Esta teoria será necessária na próxima seção.

Sejam $D=\{z \in \mathbb{C}:|z|<1\}$ e $H(D)$ a classe de todas as funções analíticas em $D$. Para $f \in H(D)$ definimos $M_{2}(f ; r)=\left\{\frac{1}{2 \pi} \int_{-\pi}^{\pi}\left|f\left(r e^{i t}\right)\right|^{2} d t\right\}^{1 / 2}$, pelo Teorema 17.6 de [38] temos que $M_{2}$ é uma função monótona crescente de $r \in[0,1)$, logo podemos definir para $f \in H(D)$

$$
\|f\|_{2}=\lim _{r \rightarrow 1} M_{2}(f ; r) .
$$

Definição 2.4 $H^{2}\left(S^{1}\right)=\left\{f \in H(D):\|f\|_{2}<\infty\right\}$.

Aplicando a desigualdade de Minkowski para $M_{2}(f ; r)$ é fácil verificar que $\|f\|_{2}$ satisfaz a desigualdade triangular, logo $H^{2}\left(S^{1}\right)$ é um espaço linear normado. Mais ainda, temos que $H^{2}\left(S^{1}\right)$ é um espaço de Banach, veja por exemplo na página 331 de [38] uma demonstração de que $H^{2}\left(S^{1}\right)$ é completo.

Veremos a seguir que $H^{2}\left(S^{1}\right)$ pode ser identificado com um subespaço de $L^{2}\left(S^{1}\right)$, $S^{1}=\left\{e^{i \theta}: \theta \in \mathbb{R}\right\}$ munido da medida $\frac{1}{2 \pi} d \theta$.

Teorema 2.5 a) Uma função $f \in H(D)$, da forma

$$
f(z)=\sum_{n=0}^{\infty} a_{n} z^{n} \quad(z \in D)
$$

está em $H^{2}\left(S^{1}\right)$ se, e somente se, $\sum_{n=0}^{\infty}\left|a_{n}\right|^{2}<\infty$; neste caso,

$$
\|f\|_{2}=\left\{\sum_{n=0}^{\infty}\left|a_{n}\right|^{2}\right\}^{2} .
$$

b) Se $f \in H^{2}\left(S^{1}\right)$, então $f$ tem limite radial $f^{*}\left(e^{i t}\right)=\lim _{r \rightarrow 1} f\left(r e^{i t}\right)$ em quase todo ponto de $S^{1} ; f^{*} \in L^{2}\left(S^{1}\right)$; o n-ésimo coeficiente de Fourier de $f^{*}$ é $a_{n}$ se $n \geq 0$ e zero se $n<0$;

$$
\lim _{r \rightarrow 1} \frac{1}{2 \pi} \int_{-\pi}^{\pi}\left|f^{*}\left(e^{i t}\right)-f\left(r e^{i t}\right)\right|^{2} d t=0
$$

Além disso $f$ é a integral de Poisson de $f^{*}$, isto é, se $z=r e^{i \theta}$ então

$$
f(z)=\frac{1}{2 \pi} \int_{-\pi}^{\pi} P_{r}(\theta-t) f^{*}\left(e^{i t}\right) d t
$$


onde $P_{r}(\theta-t)$ é o núcleo de Poisson.

c) A aplicação $f \mapsto f^{*}$ é uma isometria de $H^{2}\left(S^{1}\right)$ sobre o subespaço de $L^{2}\left(S^{1}\right)$ que consiste naquelas $g \in L^{2}\left(S^{1}\right)$ que tem $\hat{g}(n)=0$ para todo $n<0$.

Demonstração: Ver [38], Teorema 17.10.

Definição 2.6 $H^{2}(\mathbb{R})$ é o subespaço de $L^{2}(\mathbb{R})$ consistindo de todas as funções $f$ cuja extensão harmônica $F(x+i y)=\frac{1}{\pi} \int_{\mathbb{R}} \frac{y f(t)}{y^{2}+(x-t)^{2}} d t$, é analítica no semi - plano superior.

Enunciaremos agora um Teorema de Paley e Wiener que nos mostra que $F H^{2}(\mathbb{R})=$ $L^{2}\left(\mathbb{R}_{+}\right)$.

Teorema 2.7 Uma função $f \in L^{2}(\mathbb{R})$ pertence a $H^{2}(\mathbb{R})$ se, e somente se, $\hat{f}$ é nula em quase todo o ponto no eixo real negativo.

Demonstração: Ver [17], pág. 101.

Provaremos agora um resultado que relaciona $H^{2}\left(S^{1}\right)$ com $H^{2}(\mathbb{R})$.

Proposição 2.8 Seja $U: L^{2}\left(S^{1}\right) \longrightarrow L^{2}(\mathbb{R})$ onde $g \longmapsto(U g)(t)=\frac{1}{\sqrt{\pi}(1-i t)} g\left(\frac{1+i t}{1-i t}\right), t \in \mathbb{R}$. Então $U$ é um operador unitário de $L^{2}\left(S^{1}\right)$ para $L^{2}(\mathbb{R})$ tal que $U\left(H^{2}\left(S^{1}\right)\right)=H^{2}(\mathbb{R})$.

\section{Demonstração:}

Fazendo alguns cálculos, concluímos que $U$ é inversível e $U^{-1}: L^{2}(\mathbb{R}) \longrightarrow L^{2}\left(S^{1}\right)$ é dada por $h \longmapsto\left(U^{-1} h\right)(z)=\frac{2 \sqrt{\pi}}{z+1} h\left(\frac{i(z-1)}{-z-1}\right)$.

Vamos verificar que $U$ é uma isometria. Temos que

$$
\|U g\|_{L^{2}(\mathbb{R})}^{2}=\frac{1}{\pi} \int_{\mathbb{R}} \frac{1}{|1-i t|^{2}}\left|g\left(\frac{1+i t}{1-i t}\right)\right|^{2} d t .
$$

Seja $e^{i \theta}=\frac{1+i t}{1-i t}$ então $e^{i \theta} d \theta=\frac{2}{(1-i t)^{2}} d t$ sendo assim $d \theta=\frac{2}{|1-i t|^{2}} d t$. Substituindo esses valores obtemos que $\|U g\|_{L^{2}(\mathbb{R})}^{2}=\|g\|_{L^{2}\left(S^{1}\right)}^{2}$.

Verificaremos agora que $U\left(H^{2}\left(S^{1}\right)\right)=H^{2}(\mathbb{R})$. A aplicação conforme $z=\frac{1+i w}{1-i w}$ leva o semi - plano superior sobre o disco unitário, com $i$ sendo levado no zero e o zero sendo levado no um. Sejam $z_{1}=r e^{i \theta}$ e $z_{2}=r e^{i t}, r \in[0,1)$, temos que $P_{r}(\theta-t)=\operatorname{Re}\left[\frac{e^{i t}+z_{1}}{e^{i t}-z_{1}}\right]$. 
Como a aplicação conforme é bijetora temos que existem $w_{1}=x_{1}+i y_{1}$ e $w_{2}=x_{2}+i y_{2}$ tais que $z_{1}=\frac{1+i w_{1}}{1-i w_{1}}$ e $z_{2}=\frac{1+i w_{2}}{1-i w_{2}}$. Na fronteira temos $e^{i t}=\frac{1+i x_{2}}{1-i x_{2}} \operatorname{logo} \frac{1}{2 \pi} d t=\frac{1}{\pi} \frac{1}{\left(1+x_{2}^{2}\right)} d x_{2}$. Compondo a fórmula do núcleo de Poisson com a aplicação conforme obtemos $\frac{y_{1}\left(1+x_{2}^{2}\right)}{y_{1}^{2}+\left(x_{1}-x_{2}\right)^{2}}$.

Dada $f \in H^{2}\left(S^{1}\right)$ consideremos $\tilde{f}(z)=f(z) \frac{1+z}{2 \sqrt{\pi}}$. Temos que $\tilde{f} \in H^{2}\left(S^{1}\right)$. Então pelo Teorema 2.5 item b temos que $\tilde{f}\left(z_{1}\right)=\frac{1}{2 \pi} \int_{-\pi}^{\pi} P_{r}(\theta-t) \tilde{f}^{*}\left(e^{i t}\right) d t$. Compondo com a aplicação conforme temos $\tilde{f}\left(\frac{1+i w_{1}}{1-i w_{1}}\right)=\frac{1}{\pi} \int_{\mathbb{R}} \frac{y_{1}}{y_{1}^{2}+\left(x_{1}-x_{2}\right)^{2}} \tilde{f}^{*}\left(\frac{1+i x_{2}}{1-i x_{2}}\right) d x_{2}=$ $=\frac{1}{\pi} \int_{\mathbb{R}} \frac{y_{1}}{y_{1}^{2}+\left(x_{1}-x_{2}\right)^{2}} \frac{1}{\sqrt{\pi}\left(1-i x_{2}\right)} f^{*}\left(\frac{1+i x_{2}}{1-i x_{2}}\right) d x_{2}=\frac{1}{\pi} \int_{\mathbb{R}} \frac{y_{1}}{y_{1}^{2}+\left(x_{1}-x_{2}\right)^{2}}\left(U f^{*}\right)\left(x_{2}\right) d x_{2}$ que é analítica no semi-plano superior.

Portanto $U\left(H^{2}\left(S^{1}\right)\right) \subset H^{2}(\mathbb{R})$. A demonstração da volta utiliza a mesma estratégia.

Definição 2.9 Seja $P$ a projeção ortogonal de $L^{2}\left(S^{1}\right)$ sobre $H^{2}\left(S^{1}\right)$. Se $\varphi \in L^{\infty}\left(S^{1}\right)$, o operador

$$
T_{\varphi}: H^{2}\left(S^{1}\right) \longrightarrow H^{2}\left(S^{1}\right), \quad \varphi \longmapsto P(\varphi f)
$$

é chamado operador de Toeplitz com símbolo $\varphi$.

Enunciaremos agora um teorema que será utilizado na próxima seção.

Teorema 2.10 Se $\varphi \in C\left(S^{1}\right)$, então $T_{\varphi}$ é um operador de Fredholm se, e só se, $\varphi$ nunca se anula. Seja $\varphi$ um elemento inversível em $C\left(S^{1}\right)$. Então o indice de Fredholm de $T_{\varphi} e^{\prime}$ menos o número de rotação de $\varphi$, isto é,

$$
\operatorname{ind}\left(T_{\varphi}\right)=-w(\varphi)
$$

Demonstração: Ver [28], pág. 103 e 104.

\section{3 Índice de Fredholm e a K-teoria de $\mathcal{C}$}

Nesta seção e nos capítulos que seguem estudaremos a K-teoria de $C^{*}$ - álgebras. Como referência de um amplo estudo sobre $\mathrm{K}$-teoria para $C^{*}$-álgebras recomendamos os livros de B. Blackadar, K-Theory for Operator Algebras [4] e M. Rordam, F. Larsen e N. J. Laustisen, An Introduction to K-Theory for $C^{*}$ - algebras [37]. Para um breve resumo 
sobre K-teoria para $C^{*}$ - álgebras recomendamos o Apêndice C de [6]. Utilizaremos neste trabalho as notações e resultados de [4] e [37].

A K-teoria foi desenvolvida por Atiyah e Hirzebruch por volta de 1960, baseada no trabalho de Grothendieck em geometria algébrica. Ela foi introduzida como uma ferramenta na teoria de $C^{*}$ - álgebras por volta de 1970.

Resumidamente, K-teoria (para $C^{*}$ - álgebras) é um par de funtores, chamados $K_{0}$ e $K_{1}$, que a cada $C^{*}$ - álgebra $A$ associa dois grupos abelianos $K_{0}(A)$ e $K_{1}(A)$. Temos que $K_{0}$ é um grupo de diferenças formais de classes de projeções (matrizes) de tamanho arbitrário e $K_{1}$ é o grupo de classes de homotopias de matrizes unitárias de tamanho arbitrário. Uma das propriedades dos funtores $K_{0}$ e $K_{1}$ é que dada uma sequência exata curta de $C^{*}$ - álgebras

$$
0 \longrightarrow A \stackrel{\varphi}{\longrightarrow} B \stackrel{\psi}{\longrightarrow} C C \longrightarrow 0
$$

podemos associar uma sequência exata cíclica de seis termos de grupos abelianos

$$
\begin{array}{ccccc}
K_{0}(A) \stackrel{\varphi_{*}}{\longrightarrow} & K_{0}(B) \stackrel{\psi_{*}}{\longrightarrow} & K_{0}(C) \\
\uparrow \delta_{1} & & & & \\
K_{1}(C) \stackrel{\psi_{*}}{\longleftarrow} & K_{1}(B) \stackrel{\varphi_{*}}{\longleftarrow} & K_{1}(A)
\end{array}
$$

onde as setas horizontais são homomorfismos induzidos funtorialmente e a seta de conexão de $K_{1}$ para $K_{0}$ é chamada aplicação do índice e a seta de conexão de $K_{0}$ para $K_{1}$ é chamada aplicação exponencial.

Vimos na seção 1 que $\mathrm{C} / \mathcal{K}_{\mathbb{R}}$ é isomorfo a $C\left(M_{C}\right)$. Usando que $M_{C}$ é homeomorfo a $S^{1}$ temos que $K_{i}\left(\mathrm{e} / \mathcal{K}_{\mathbb{R}}\right) \cong K_{i}\left(C\left(S^{1}\right)\right), i=0,1$. Pelo exemplo 11.3 .3 de [37] sabemos que $K_{i}\left(C\left(S^{1}\right)\right) \cong \mathbb{Z}, i=0,1$, onde a classe [.] da função $f: S^{1} \longrightarrow \mathbb{C}$ definida por $f(z)=z$ gera $K_{1}\left(C\left(S^{1}\right)\right)$ e a classe [.] da função $g: S^{1} \longrightarrow \mathbb{C}$ definida por $g(z)=1$ gera $K_{0}\left(C\left(S^{1}\right)\right)$. Pelo exemplo 11.3.4 de [37] temos que

$$
\begin{aligned}
\triangle: K_{1}\left(C\left(S^{1}\right)\right) & \longrightarrow \pi^{1}\left(S^{1}\right) \\
{[u]_{1} } & \longmapsto<\operatorname{Det}(u)>,
\end{aligned}
$$

onde $\pi^{1}\left(S^{1}\right)$ é o grupo fundamental de $S^{1}$ e $u$ é um unitário é um isomorfismo. Pelo 
exemplo 8.3.2 de [37] temos que

$$
\begin{aligned}
w: \pi^{1}\left(S^{1}\right) & \longrightarrow \mathbb{Z} \\
<\lambda> & \longrightarrow w(\lambda),
\end{aligned}
$$

onde $\lambda \in C\left(S^{1}, S^{1}\right)$ é um isomorfismo e satisfaz a seguinte propriedade $u$ é homotópico a $v$ se, e só se, $w(u)=w(v)$, $u$ e $v \in C\left(S^{1}, S^{1}\right)$.

Logo

$$
\begin{aligned}
w \circ \Delta: K_{1}\left(C\left(S^{1}\right)\right) & \longrightarrow \\
{[u]_{1} } & \longmapsto w(\operatorname{Det}(u))
\end{aligned}
$$

é um isomorfismo. Com isto temos que a classe de qualquer $f \in C\left(S^{1}, S^{1}\right)$ que tenha $w(f)=1$ ou -1 gera $K_{1}\left(C\left(S^{1}\right)\right)$.

Fixando um homeomorfismo $h$ de $M_{C}$ para $S^{1}$ temos que $[1]_{0}$ gera $K_{0}\left(C\left(M_{C}\right)\right)$ e $[h]_{1}$ gera $K_{1}\left(C\left(M_{C}\right)\right)$.

Usando o $\sigma$-símbolo temos que $\left[[I d]_{\mathcal{K}_{\mathbb{R}}}\right]_{0}$ gera $K_{0}\left(\mathcal{C} / \mathcal{K}_{\mathbb{R}}\right)$ e $\left[\left[e^{2 \pi i c(M)} b(D)+c(D)\right]_{\mathcal{K}_{\mathbb{R}}}\right]_{1}$ onde $b, c \in C S(\mathbb{R})$ são tais que $b$ é crescente com $b(x)=0$, se $x \leq-1$ e $b(x)=1$, se $x \geq 1$ e $c$ é decrescente com $c(x)=0$, se $x \geq 1$ e $c(x)=1$, se $x \leq-1, b+c \equiv 1$ gera $K_{1}\left(\mathrm{C} / \mathcal{K}_{\mathbb{R}}\right)$, isto é,

$$
\begin{gathered}
K_{0}\left(\mathcal{C} / \mathcal{K}_{\mathbb{R}}\right)=\mathbb{Z}\left[[I d]_{\mathcal{K}_{\mathbb{R}}}\right]_{0} \quad \mathrm{e} \\
K_{1}\left(\mathcal{C} / \mathcal{K}_{\mathbb{R}}\right)=\mathbb{Z}\left[\left[e^{2 \pi i c(M)} b(D)+c(D)\right]_{\mathcal{K}_{\mathbb{R}}}\right]_{1}
\end{gathered}
$$

Lema 2.11 Seja $T \in \mathcal{C}$. Então $T$ é um operador de Fredholm se, e somente se, $\sigma_{T}$ é inversível.

\section{Demonstração:}

Como $\mathcal{C} / \mathcal{K}_{\mathbb{R}}$ é uma $C^{*}$ - subálgebra de $\mathcal{L}\left(L^{2}(\mathbb{R})\right) / \mathcal{K}_{\mathbb{R}}$, segue do Teorema 2.1 .11 de [28] que $[T]_{\mathcal{K}_{\mathbb{R}}} \in \mathcal{C} / \mathcal{K}_{\mathbb{R}}$ é inversível se, e somente se, $[T]_{\mathcal{K}_{\mathbb{R}}} \in \mathcal{L}\left(L^{2}(\mathbb{R})\right) / \mathcal{K}_{\mathbb{R}}$ é inversível. Pelo Teorema 1.4.16 de [28] temos que $[T]_{\mathcal{K}_{\mathbb{R}}} \in \mathcal{L}\left(L^{2}(\mathbb{R})\right) / \mathcal{K}_{\mathbb{R}}$ é inversível se, e somente se, $T$ é de Fredholm. Por outro lado, segue do Teorema 2.3 que $[T]_{\mathcal{K}_{\mathbb{R}}} \in \mathcal{C} / \mathcal{K}_{\mathbb{R}}$ é inversível se, e só se, $\sigma_{T}(x, \xi) \neq 0$ para todo $(x, \xi) \in M_{C}$.

Sejam $\chi$ a função característica da semi-reta real positiva e $\phi \in C S(\mathbb{R})$ tal que $\phi(+\infty)=\phi(-\infty)=1, \phi$ não nula e $w(\phi)=-1$. Pelo Teorema 2.7 temos que $\chi(D) \phi(M) \chi(D)$ 
é equivalente a um operador de Toeplitz em $H^{2}(\mathbb{R})$, logo unitariamente equivalente a um operador de Toeplitz em $H^{2}\left(S^{1}\right)$, pela Proposição 2.8. Então pelo Teorema 2.10 temos que $\chi(D) \phi(M) \chi(D)$ é um operador de Fredholm com índice 1. Logo $\chi(D) \phi(M) \chi(D) \oplus$ $I d_{H^{2}(\mathbb{R}) \perp}=\chi(D) \phi(M) \chi(D)+(I d-\chi(D))$ é um operador de Fredholm com índice 1.

Lema 2.12 O operador $(I d-\chi(D)) \phi(M) \chi(D)$ é compacto.

\section{Demonstração:}

Pela Proposição 2.8 e pelo Teorema 2.7 temos que $U^{-1} \chi(D) U=P$ onde $P$ é a projeção ortogonal em $H^{2}\left(S^{1}\right)$. Temos também que $U^{-1} \phi(M) U=\tilde{\phi}(M)$ onde $\tilde{\phi}(z)=$ $\phi\left(\frac{z-1}{i(z+1)}\right)$.

Suponha $\phi(t)=1$ se $|t|>1$, então $\tilde{\phi}(z) \equiv 1$ em uma vizinhança de -1 . Portanto $\tilde{\phi} \in C^{\infty}\left(S^{1}\right)$. Logo, $U^{-1}(I d-\chi(D)) \phi(M) \chi(D) U=(I d-P) \tilde{\phi}(M) P$ com $P$ a projeção sobre $H^{2}\left(S^{1}\right)$ e $\tilde{\phi} \in C^{\infty}\left(S^{1}\right)$.

Consideremos $\left(e_{k}\right)_{k \in \mathbb{Z}}$ base ortonormal de $L^{2}\left(S^{1}\right)$ onde $e_{k}(z)=z^{k}$. Temos que $(I d-P) e_{n}(M) P e_{k}=0 \quad$ se $\quad k<0 \quad$ e $\quad(I d-P) e_{n}(M) P e_{k}=(I d-P) e_{k+n} \quad$ se $\quad k \geq 0$

Mas $(I d-P) e_{k+n}=0$ se $k+n \geq 0$. Vamos estudar então o que acontece para $n \geq 0$ e $n<0$.

Se $n \geq 0$ então $k+n \geq 0$ para todo $k \geq 0, \operatorname{logo}(I d-P) e_{k+n}=0$.

Se $n<0$ teremos $k+n \geq 0$ se, e somente se, $k \geq-n$. Então $(I d-P) e_{k+n}=0$ para todo $k \geq-n$ e $(I d-P) e_{k+n}=e_{k+n}$ caso contrário. Logo para n fixo $\operatorname{Im}\left[(I d-P) e_{n} P\right]$ é gerada por $\left\{e_{k}: 0 \leq k \leq-n\right\}$.

Com isto temos que $(I d-P) p_{n}(M) P$ é um operador de posto finito, logo compacto. Logo se $p$ é um polinômio temos que $(I d-P) p(M) P$ é compacto. Pelo Teorema de Stone-Weierstrass temos que existem polinômios $p_{n}$ convergindo uniformemente para $\tilde{\phi}$ então

$$
\left\|(I d-P) \tilde{\phi}(M) P-(I d-P) p_{n}(M) P\right\| \leq\left\|\tilde{\phi}(M)-p_{n}(M)\right\|=\left\|\tilde{\phi}-p_{n}\right\|_{\infty} \longrightarrow 0 .
$$

Como $\mathcal{K}\left(L^{2}\left(S^{1}\right)\right)$ é fechado em $\mathcal{L}\left(L^{2}\left(S^{1}\right)\right)$ temos que $(I d-P) \tilde{\phi}(M I) P$ é compacto em $L^{2}\left(S^{1}\right)$. Logo $(I d-\chi(D)) \phi(M) \chi(D)$ é compacto em $L^{2}(\mathbb{R})$. 
Teorema 2.13 Seja $T \in \mathcal{C}$ um operador de Fredholm, temos que ind $(T)=\delta_{1}\left(\left[[T]_{\mathcal{K}_{\mathbb{R}}}\right]_{1}\right)=$ $w\left(\sigma_{T}\right)$, onde $\delta_{1}$ é a aplicação do índice da sequência de K-teoria associada a sequência exata curta abaixo.

$$
0 \longrightarrow \mathcal{K}_{\mathbb{R}} \stackrel{i}{\longrightarrow} \mathrm{e} \stackrel{\pi}{\longrightarrow} \mathrm{e} / \mathcal{K}_{\mathbb{R}} \longrightarrow 0
$$

\section{Demonstração:}

Vamos considerar as seguintes sequências exatas curtas

$$
\begin{gathered}
0 \longrightarrow \mathcal{K}_{\mathbb{R}} \stackrel{i}{\longrightarrow} \mathcal{P} \stackrel{\pi}{\longrightarrow} \mathcal{C} / \mathcal{K}_{\mathbb{R}} \longrightarrow 0 \\
0 \longrightarrow \mathcal{K}_{\mathbb{R}} \stackrel{i}{\longrightarrow} \mathcal{L}\left(L^{2}(\mathbb{R})\right) \stackrel{\pi}{\longrightarrow} \mathcal{L}\left(L^{2}(\mathbb{R})\right) / \mathcal{K}_{\mathbb{R}} \longrightarrow 0
\end{gathered}
$$

onde $i$ é a inclusão e $\pi$ a projeção canônica.

Sabemos pela Proposição 9.4 .2 de [37] que o índice, $\bar{\delta}_{1}$, para a sequência exata curta

$$
0 \longrightarrow \mathcal{K}_{\mathbb{R}} \stackrel{i}{\longrightarrow} \mathcal{L}\left(L^{2}(\mathbb{R})\right) \stackrel{\pi}{\longrightarrow} \mathcal{L}\left(L^{2}(\mathbb{R})\right) / \mathcal{K}_{\mathbb{R}} \longrightarrow 0
$$

é o índice de Fredholm, isto é $\operatorname{ind}(T)=\bar{\delta}_{1}\left(\left[[T]_{\mathcal{K}_{\mathbb{R}}}\right]_{1}\right)$.

Consideremos o diagrama comutativo

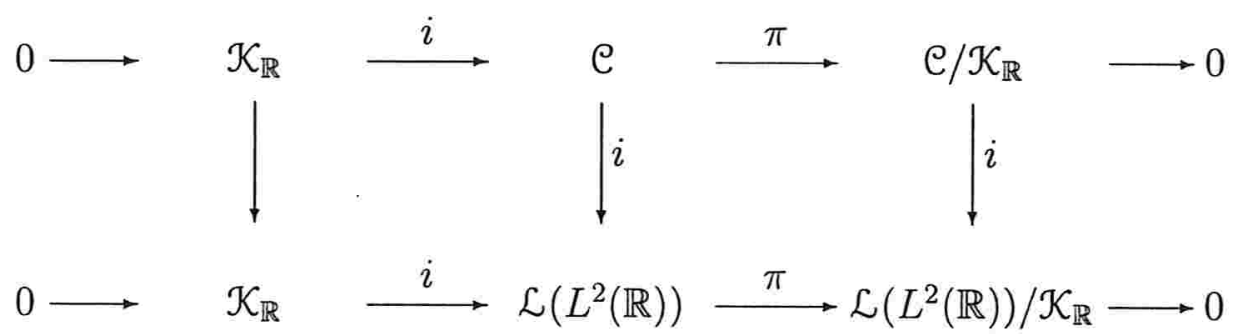

Pela naturalidade da aplicação do índice, veja Proposição 9.1.5 de [37], temos que o diagrama abaixo comuta

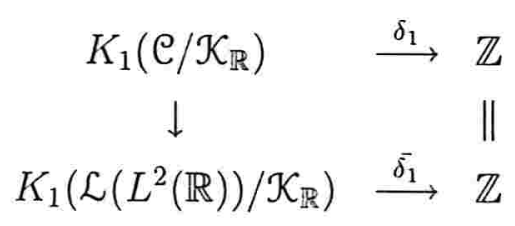

Logo $\delta_{1}$ é o índice de Fredholm, ou seja $\operatorname{ind}(T)=\delta_{1}\left(\left[[T]_{\mathcal{K}_{\mathrm{R}}}\right]_{1}\right)$. Vamos verificar agora que $\operatorname{ind}(T)=w\left(\sigma_{T}\right)$. 
Consideremos $S=\chi(D) \phi(M) \chi(D)+(I d-\chi(D))+(I d-\chi(D)) \phi(M) \chi(D)=$ $\phi(M) \chi(D)+(I d-\chi(D))$. Como vimos no Lema 2.12 que $(I d-\chi(D)) \phi(M) \chi(D)$ é compacto, temos que $S$ é um operador de Fredholm com índice 1, pela observação antes do Lema 2.12 .

Seja $T^{\prime}=\phi(M) b(D)+(I d-b(D))$ com $b$ contínua crescente com $b(x)=0$ para $x \leq-1$ e $b(x)=1$ para $x \geq 1$. Temos $T^{\prime} \in \mathrm{C}$ e $T^{\prime}-S=(\phi-1)(M)(b-\chi)(D)$ logo $T^{\prime}-S$ é compacto pela Observação $1.141 / 2$. Portanto $T^{\prime}=S+K, K$ compacto, logo $T^{\prime}$ é Fredholm e ind $\left(T^{\prime}\right)=1$ e temos também que $w\left(\sigma_{T^{\prime}}\right)=1$. Concluimos assim que $\operatorname{ind}(T)=\delta_{1}\left(\left[[T]_{\mathcal{K}_{\mathbb{R}}}\right]_{1}\right)=w\left(\sigma_{T}\right), T \in \mathcal{C}$ um operador de Fredholm.

Proposição 2.14 Seja $\pi: \mathcal{C} \longrightarrow \mathcal{C} / \mathcal{K}_{\mathbb{R}}$ a projeção canônica. Então $\pi_{*}: K_{0}(\mathcal{C}) \longrightarrow$ $K_{0}\left(\mathfrak{e} / \mathcal{K}_{\mathbb{R}}\right)$ é um isomorfismo. Além disso, $K_{1}(\mathfrak{C})=0$.

\section{Demonstração:}

Consideremos a sequência exata curta

$$
0 \longrightarrow \mathcal{K}_{\mathbb{R}} \stackrel{i}{\longrightarrow} \mathcal{e} \stackrel{\pi}{\longrightarrow} \mathcal{e} / \mathcal{K}_{\mathbb{R}} \longrightarrow 0
$$

e sua sequência exata cíclica de seis termos



Pelo corolário 6.4.2 e pelo exemplo 8.2.9 de [37] temos que $K_{0}\left(\mathcal{K}_{\mathbb{R}}\right)=\mathbb{Z}$ e $K_{1}\left(\mathcal{K}_{\mathbb{R}}\right)=0$. Substituindo então as $\mathrm{K}$ - teorias conhecidas temos

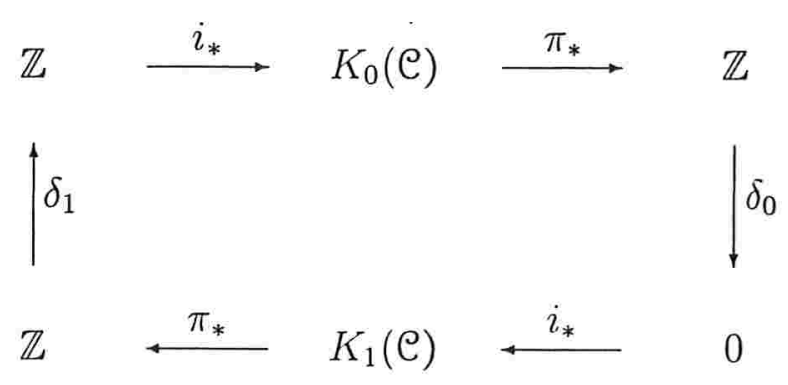


Seja $T^{\prime}=\phi(M) b(D)+(I d-b(D))$ o operador do Teorema 2.13 , temos que $\left[\left[T^{\prime}\right]_{\mathcal{K}_{\mathrm{R}}}\right]_{1} \in$ $K_{1}\left(\mathrm{e} / \mathcal{K}_{\mathbb{R}}\right)$ e já vimos que $\delta_{1}\left(\left[\left[T^{\prime}\right]_{\mathcal{K}_{\mathbb{R}}}\right]_{1}\right)=1$, logo $\delta_{1}$ é um isomorfismo. Portanto $\pi_{*}$ : $K_{0}(\mathrm{C}) \longrightarrow \mathbb{Z}$ é isomorfismo e $K_{1}(\mathrm{C})=0$. 


\section{Capítulo 3}

\section{K-teoria de $\mathcal{A}$}

Neste capítulo calcularemos a K-teoria da $C^{*}$ - álgebra $\mathcal{A}$ utilizando a seguinte estratégia. Primeiro calcularemos a K-teoria de $\mathcal{A} / \mathcal{E}$ e $\mathcal{E} / \mathcal{K}_{\mathbb{R}}$ e para isto utilizaremos os isomorfismos $\varphi: \mathcal{A} / \mathcal{E} \longrightarrow C\left(M_{A}\right)$ e $\gamma: \mathcal{E} / \mathcal{K}_{\mathbb{R}} \longrightarrow C\left(M_{S L}, \mathcal{K}_{\mathbb{Z}}\right)$, estudados no Capítulo 1 . De posse dessas K-teorias e utilizando a seguinte sequência exata curta

$$
0 \longrightarrow \mathcal{E} / \mathcal{K}_{\mathbb{R}} \longrightarrow \mathcal{A} / \mathcal{K}_{\mathbb{R}} \longrightarrow \mathcal{A} / \mathcal{E} \longrightarrow 0
$$

calcularemos a K-teoria de $\mathcal{A} / \mathcal{K}_{\mathbb{R}}$. Por último utilizamos a sequência exata curta abaixo para o cálculo da K-teoria de $\mathcal{A}$

$$
0 \longrightarrow \mathcal{K}_{\mathbb{R}} \longrightarrow \mathcal{A} \longrightarrow \mathcal{A} / \mathcal{K}_{\mathbb{R}} \longrightarrow 0
$$

\section{$3.1 \quad$ A K-teoria de $\mathcal{A} / \mathcal{E}$ e $\mathcal{E} / \mathcal{K}_{\mathbb{R}}$}

Começaremos o nosso trabalho por descrever geradores para $K_{i}\left(C\left(M_{\sharp}\right)\right), i=0,1$, já que pelo Teorema $1.17 M_{A}=M_{\sharp} \times\{-\infty,+\infty\}$ e pelo Teorema de Gelfand temos $\varphi: \mathcal{A} / \mathcal{E} \longrightarrow C\left(M_{A}\right)$ isomorfismo. Pelo Lema 1.10 podemos considerar $M_{\sharp}=S_{-}^{1} \cup \mathbb{R} \cup S_{+}^{1}$, onde $S_{+}^{1}=S^{1} \times\{+\infty\}$ e $S_{-}^{1}=S^{1} \times\{-\infty\}$.

Proposição 3.1 Seja $\psi: C\left(M_{\sharp}\right) \longrightarrow C\left(S^{1} \times\{-\infty,+\infty\}\right)$ a restrição das $f \in C\left(M_{\sharp}\right)$ a $S_{+}^{1} \cup S_{-}^{1}$. Para $i=0,1 \psi_{*}: K_{i}\left(C\left(M_{\sharp}\right)\right) \longrightarrow K_{i}\left(C\left(S^{1} \times\{-\infty,+\infty\}\right)\right)$ é injetora. $A$ 
imagem de $\psi_{*}: K_{0}\left(C\left(M_{\sharp}\right)\right) \longrightarrow K_{0}\left(C\left(S^{1} \times\{-\infty,+\infty\}\right)\right)$ é isomorfa a $\mathbb{Z}$ e a imagem de $\psi_{*}: K_{1}\left(C\left(M_{\sharp}\right)\right) \longrightarrow K_{1}\left(C\left(S^{1} \times\{-\infty,+\infty\}\right)\right)$ é isomorfa a $\mathbb{Z} \oplus \mathbb{Z}$. Assim $K_{0}\left(C\left(M_{\sharp}\right)\right) \cong \mathbb{Z}$ e $K_{1}\left(C\left(M_{\sharp}\right)\right) \cong \mathbb{Z} \oplus \mathbb{Z}$.

\section{Demonstração:}

Consideremos a sequência exata curta

$$
0 \longrightarrow C_{0}(\mathbb{R}) \stackrel{\varphi}{\longrightarrow} C\left(M_{\sharp}\right) \stackrel{\psi}{\longrightarrow} C\left(S^{1} \times\{-\infty,+\infty\}\right) \longrightarrow 0
$$

onde $\varphi$ é a inclusão e $\psi$ é a restrição.

Sabemos que esta sequência exata curta induz a sequência exata cíclica de seis termos em K-teoria

$$
\begin{gathered}
K_{0}\left(C_{0}(\mathbb{R})\right) \stackrel{\varphi_{*}}{\longrightarrow} K_{0}\left(C\left(M_{\sharp}\right)\right) \stackrel{\psi_{*}}{\longrightarrow} K_{0}\left(C\left(S^{1} \times\{-\infty,+\infty\}\right)\right) \\
\left\{\delta_{1}\right.
\end{gathered}
$$

Pelo exemplo 11.3.2 de [37] sabemos que $K_{0}\left(C_{0}(\mathbb{R})\right)=0$ e $K_{1}\left(C_{0}(\mathbb{R})\right)=\mathbb{Z}$. Assim como pelo exemplo 11.3.3 de [37] e o fato de $C\left(S^{1} \times\{-\infty,+\infty\}\right)$ ser isomorfo a $C\left(S^{1}\right) \oplus$ $C\left(S^{1}\right)$ temos para $i=0,1$ que $K_{i}\left(C\left(S^{1} \times\{-\infty,+\infty\}\right)\right) \cong \mathbb{Z} \oplus \mathbb{Z}$ onde a classe [.]o das funções $k, \tilde{k}: S^{1} \times\{-\infty,+\infty\} \rightarrow \mathbb{C} \operatorname{com} k(z,+\infty)=1 ; k(z,-\infty)=0 ; \tilde{k}(z,+\infty)=0$; $\tilde{k}(z,-\infty)=1$ geram $K_{0}\left(C\left(S^{1} \times\{-\infty,+\infty\}\right)\right)$. E a classe [.] $]_{1}$ das funções $l, \tilde{l}: S^{1} \times$ $\{-\infty,+\infty\} \rightarrow \mathbb{C} \operatorname{com} l(z,+\infty)=z ; l(z,-\infty)=1 ; \tilde{l}(z,+\infty)=1 ; \tilde{l}(z,-\infty)=z$ geram $K_{1}\left(C\left(S^{1} \times\{-\infty,+\infty\}\right)\right)$.

Substituindo na sequência exata cíclica de seis termos as informações acima temos que $\delta_{1} \equiv 0$. Só nos falta calcular $\delta_{0}$ para obter a K-teoria de $C\left(M_{\sharp}\right)$.

Pela Proposição 12.2 .2 de [37] sabemos que precisamos de funções $g$ e $\tilde{g} \in C\left(M_{\sharp}\right)$ reais tais que $\psi(g)=k$ e $\psi(\tilde{g})=\tilde{k}$.

Consideremos $b: M_{\sharp} \rightarrow \mathbb{R} \operatorname{com} b \equiv 0$ em $S_{-}^{1} ; b \equiv 1$ em $S_{+}^{1} ; b(x)=0$ para $x \leq-1 \mathrm{e}$ $b(x)=1$ para $x \geq 1$.

E também $c: M_{\sharp} \rightarrow \mathbb{R} \operatorname{com} c \equiv 1$ em $S_{-}^{1} ; c \equiv 0$ em $S_{+}^{1} ; c(x)=1$ para $x \leq-1$ e $c(x)=0$ 
para $x \geq 1$. As funções $b$ e $c$ são extensões contínuas das funções $b$ e $c$ definidas na página 47, mas utilizaremos a mesma notação.

Temos que $\psi(b)=k$ e $\psi(c)=\tilde{k}$, então pela Proposição 12.2 .2 de [37] temos que existem (e são únicos) elementos $u$ e $\tilde{u} \in \mathcal{U}\left(\widetilde{C_{0}(\mathbb{R})}\right)$ tais que $\bar{\varphi}(u)=e^{2 \pi i b}$ e $\bar{\varphi}(\tilde{u})=e^{2 \pi i c}$. Mas como $\widetilde{C_{0}(\mathbb{R})} \subset C\left(M_{\sharp}\right)$ temos que $\varphi=\bar{\varphi}$, logo $u=e^{2 \pi i b}$ e $\tilde{u}=e^{2 \pi i c}$.

Portanto $\delta_{0}\left([k]_{0}\right)=-[u]_{1}$ e $\delta_{0}\left([\tilde{k}]_{0}\right)=-[\tilde{u}]_{1}$ onde $[u]_{1}$ e $[\tilde{u}]_{1}$ pertencem a $K_{1}\left(C_{0}(\mathbb{R})\right)$. Sabemos que $K_{1}\left(C_{0}(\mathbb{R})\right)$ é isomorfo a $K_{1}\left(C\left(S^{1}\right)\right)$ e temos que o número de rotação induz um isomorfismo de $K_{1}\left(C\left(S^{1}\right)\right)$ para $\mathbb{Z}$.

Calculando o número de rotação de $u$ e $\tilde{u}$ temos que $w(u)=1$ e $w(\tilde{u})=-1$. Logo com respeito aos isomorfismos $K_{0}\left(C\left(S^{1} \times\{-\infty,+\infty\}\right)\right)=\mathbb{Z}[k]_{0} \oplus \mathbb{Z}[\tilde{k}]_{0}$ e $K_{1}\left(C\left(S^{1} \times\right.\right.$ $\{-\infty,+\infty\}))=\mathbb{Z}[l]_{1} \oplus \mathbb{Z}[\tilde{l}]_{1}$ discutidos acima, podemos escrever $\delta_{0}(1,0)=-1$ e $\delta_{0}(0,1)=$ 1 , consequentemente $\delta_{0}$ é sobrejetora e $\delta_{0}(x, y)=-x+y$.

Segue da exatidão da sequência exata cíclica de seis termos que $\operatorname{Im} \psi_{*}=\operatorname{Ker} \delta_{0} \cong$ $\mathbb{Z}, \operatorname{Ker} \psi_{*}=\operatorname{Im} \varphi_{*}=0, \operatorname{Im} \psi_{*}=\operatorname{Ker} \delta_{1} \cong \mathbb{Z} \oplus \mathbb{Z}$ e $\operatorname{Ker} \psi_{*}=\operatorname{Im} \varphi_{*}=0$. Portanto $K_{0}\left(C\left(M_{\sharp}\right)\right) \cong \mathbb{Z}$ e $K_{1}\left(C\left(M_{\sharp}\right)\right) \cong \mathbb{Z} \oplus \mathbb{Z}$.

Usando os isomorfismos da Proposição acima podemos verificar que

$$
K_{0}\left(C\left(M_{\sharp}\right)\right)=\mathbb{Z}[1]_{0} .
$$

Sejam $S^{1}=\left\{e^{i x}: x \in \mathbb{R}\right\}, L$ e $\tilde{L} \in C\left(M_{\sharp}\right)$ tais que:

$$
L\left(e^{i x},+\infty\right)=e^{i x} \text { em } S_{+}^{1} ; L\left(e^{i x},-\infty\right)=1 \text { em } S_{-}^{1} ; L(x)=e^{i x} \text {, se } x \geq 0 \text { e } L(x)=1 \text {, se }
$$
$x<0$ e,

$\tilde{L}\left(e^{i x},+\infty\right)=1$ em $S_{+}^{1} ; \tilde{L}\left(e^{i x},-\infty\right)=e^{i x}$ em $S_{-}^{1} ; \tilde{L}(x)=1$, se $x>0$ e $\tilde{L}(x)=e^{i x}$, se $x \leq 0$.

Consideremos a restrição de $L$ à reta. Então temos que $b(x) e^{i x}+c(x)-L(x) \in C_{0}(\mathbb{R})$, onde $b, c \in C S(\mathbb{R})$ são tais que $b$ é crescente com $b(x)=0$, se $x \leq-1$ e $b(x)=1$, se $x \geq 1$ e $c$ é decrescente $\operatorname{com} c(x)=0$, se $x \geq 1$ e $c(x)=1$, se $x \leq-1, b+c \equiv 1$. Logo $L(M)=b(M) e^{i M}+c(M)-a_{0}(M)$, onde $a_{0} \in C_{0}(\mathbb{R})$. O mesmo vale para $\tilde{L}$, logo $\tilde{L}(M)=b(M)+c(M) e^{i M}-b_{0}(M)$. 
Com o que acabamos de provar, concluímos que $L, \tilde{L}$ estão em $\mathcal{A}^{\sharp}$ (veja definição na seção 1.2). Usando novamente o isomorfismo da Proposição acima concluímos que

$$
K_{1}\left(C\left(M_{\sharp}\right)\right)=\mathbb{Z}[L]_{1} \oplus \mathbb{Z}[\tilde{L}]_{1}
$$

Proposição $3.2 K_{0}\left(C\left(M_{A}\right)\right)$ é isomorfo $a \mathbb{Z} \oplus \mathbb{Z}$ e $K_{1}\left(C\left(M_{A}\right)\right)$ é isomorfo a $\mathbb{Z} \oplus \mathbb{Z} \oplus \mathbb{Z} \oplus \mathbb{Z}$.

\section{Demonstração:}

Sabemos pelo Teorema 1.17 que $M_{A}=M_{\sharp} \times\{-\infty,+\infty\}$, então consideremos o isomorfismo

$$
\begin{array}{ccc}
C\left(M_{\sharp} \times\{-\infty,+\infty\}\right) & \longrightarrow & C\left(M_{\sharp}\right) \oplus C\left(M_{\sharp}\right) \\
f(z, \pm \infty) & \longmapsto & (f(z,+\infty), f(z,-\infty)) .
\end{array}
$$

Logo temos que $K_{i}\left(C\left(M_{A}\right)\right) \cong K_{i}\left(C\left(M_{\sharp}\right) \oplus C\left(M_{\sharp}\right)\right), i=0,1$.

Pelas Proposições 4.3.4 e 8.2.6 de [37] temos que $K_{i}\left(C\left(M_{\sharp}\right) \oplus C\left(M_{\sharp}\right)\right) \cong K_{i}\left(C\left(M_{\sharp}\right)\right) \oplus$ $K_{i}\left(C\left(M_{\sharp}\right)\right)$, logo $K_{i}\left(C\left(M_{A}\right)\right) \cong K_{i}\left(C\left(M_{\sharp}\right)\right) \oplus K_{i}\left(C\left(M_{\sharp}\right)\right), i=0,1$. Consideremos as funções:

$$
\begin{gathered}
f: M_{\sharp} \times\{-\infty,+\infty\} \longrightarrow \mathbb{C} \operatorname{com} f(z,+\infty)=1 \text { e } f(z,-\infty)=0, \\
\tilde{f}: M_{\sharp} \times\{-\infty,+\infty\} \longrightarrow \mathbb{C} \operatorname{com} \tilde{f}(z,+\infty)=0 \text { e } \tilde{f}(z,-\infty)=1, \\
w_{i}: M_{\sharp} \times\{-\infty,+\infty\} \longrightarrow \mathbb{C}, i=1, . ., 4, \text { com } \\
w_{1}(z,+\infty)=L(z) \text { e } w_{1}(z,-\infty)=1, \\
w_{2}(z,+\infty)=\tilde{L}(z) \text { e } w_{2}(z,-\infty)=1, \\
w_{3}(z,+\infty)=1 \text { e } w_{3}(z,-\infty)=L(z), \\
w_{4}(z,+\infty)=1 \text { e } w_{4}(z,-\infty)=\tilde{L}(z), z \in M_{\sharp} .
\end{gathered}
$$

Utilizando o isomorfismo acima temos que

$$
\begin{gathered}
K_{0}\left(C\left(M_{A}\right)\right)=\mathbb{Z}[f]_{0} \oplus \mathbb{Z}[\tilde{f}]_{0} \quad \text { e } \\
K_{1}\left(C\left(M_{A}\right)\right)=\mathbb{Z}\left[w_{1}\right]_{1} \oplus \mathbb{Z}\left[w_{2}\right]_{1} \oplus \mathbb{Z}\left[w_{3}\right]_{1} \oplus \mathbb{Z}\left[w_{4}\right]_{1} .
\end{gathered}
$$

Agora utilizando o $\sigma$-símbolo definido no Teorema 1.17 concluímos que

$$
K_{0}(\mathcal{A} / \mathcal{E})=\mathbb{Z}\left[[b(D)]_{\mathcal{E}}\right]_{0} \oplus \mathbb{Z}\left[[c(D)]_{\mathcal{E}}\right]_{0} \quad \mathrm{e}
$$




$$
K_{1}(\mathcal{A} / \mathcal{E})=\mathbb{Z}\left[\left[A_{1}\right]_{\varepsilon}\right]_{1} \oplus \mathbb{Z}\left[\left[A_{2}\right]_{\varepsilon}\right]_{1} \oplus \mathbb{Z}\left[\left[A_{3}\right]_{\varepsilon}\right]_{\mathrm{i}} \oplus \mathbb{Z}\left[\left[A_{4}\right]_{\varepsilon}\right]_{1}
$$

onde $A_{1}=L(M) b(D)+c(D), A_{2}=\tilde{L}(M) b(D)+c(D), A_{3}=b(D)+L(M) c(D)$ e $A_{4}=$ $b(D)+\tilde{L}(M) c(D)$ com $b, c$ as mesmas funções definidas na página 54 .

Proposição 3.30 isomorfismo da Proposição 1.24, $\gamma: \mathcal{E} / \mathcal{K}_{\mathbb{R}} \longrightarrow C\left(M_{S L}, \mathcal{K}_{\mathbb{Z}}\right)$, induz o isomorfismo $K_{i}\left(\mathcal{E} / \mathcal{K}_{\mathbb{R}}\right) \cong \mathbb{Z} \oplus \mathbb{Z}, i=0,1$.

\section{Demonstração:}

Usando o isomorfismo $\gamma: \mathcal{E} / \mathcal{K}_{\mathbb{R}} \longrightarrow C\left(M_{S L}, \mathcal{K}_{\mathbb{Z}}\right)$ temos que $K_{i}\left(\mathcal{E} / \mathcal{K}_{\mathbb{R}}\right)$ é isomorfo a $K_{i}\left(C\left(M_{S L}, \mathcal{K}_{\mathbb{Z}}\right)\right), i=0,1$ onde $M_{S L}=S^{1} \times\{-1,1\}$.

Então vamos calcular a K-teoria de $C\left(M_{S L}, \mathcal{K}_{\mathbb{Z}}\right)$ para conhecermos a K-teoria de $\mathcal{E} / \mathcal{K}_{\mathbb{R}}$. Começaremos por calcular a K-teoria de $C\left(S^{1}, \mathcal{K}_{\mathbb{Z}}\right)$, por isso consideremos a seguinte sequência exata curta cindida

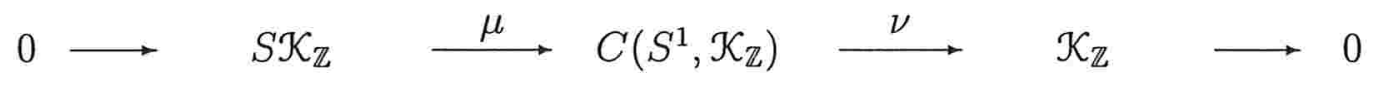

onde $\nu(f(z))=f(1)$ e $S \mathcal{K}_{\mathbb{Z}}$ denota a suspensão de $\mathcal{K}_{\mathbb{Z}}$, isto é $S \mathcal{K}_{\mathbb{Z}}=\left\{f: S^{1} \rightarrow \mathcal{K}_{\mathbb{Z}}\right.$ : $f(1)=0\}$.

E sua sequência exata cíclica de seis termos

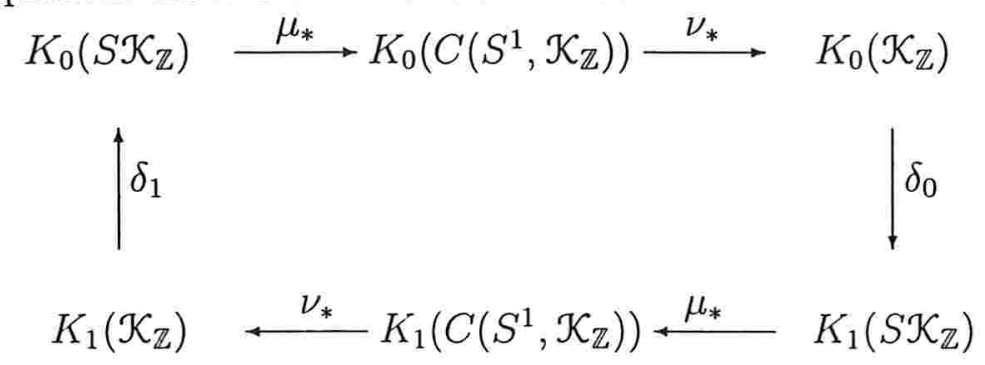

Como a sequência é cindida temos que $\delta_{0}=\delta_{1}=0$. Segue da exatidão da sequência exata cíclica de seis termos que $\nu_{*}: K_{0}\left(C\left(S^{1}, \mathcal{K}_{\mathbb{Z}}\right)\right) \rightarrow K_{0}\left(\mathcal{K}_{\mathbb{Z}}\right)=\mathbb{Z}[E]_{0}$, onde $E$ é qualquer projeção de posto $1 \mathrm{em} \mathcal{L}\left(L^{2}(\mathbb{Z})\right)$ é isomorfismo e que $\mu_{*}: K_{1}\left(S \mathcal{K}_{\mathbb{Z}}\right) \rightarrow K_{1}\left(C\left(S^{1}, \mathcal{K}_{\mathbb{Z}}\right)\right)$ é isomorfismo.

Usando o isomorfismo da periodicidade de Bott temos que $K_{1}\left(S \mathcal{K}_{\mathbb{Z}}\right)=\mathbb{Z}[1+(z-1) E]_{1}$ $\operatorname{logo} K_{1}\left(C\left(S^{1}, \mathcal{K}_{\mathbb{Z}}\right)\right)=\mathbb{Z}[1+(z-1) E]_{1}, z$ denotando a função identidade em $S^{1}$. 
Considerando o isomorfismo .

$$
\begin{array}{cc}
C\left(S^{1} \times\{-1,1\}, \mathcal{K}_{\mathbb{Z}}\right) & \longrightarrow C\left(S^{1}, \mathcal{K}_{\mathbb{Z}}\right) \oplus C\left(S^{1}, \mathcal{K}_{\mathbb{Z}}\right) \\
f(z, \pm 1) & \longmapsto(f(z, 1), f(z,-1)),
\end{array}
$$

temos que $K_{0}\left(C\left(M_{S L}, \mathcal{K}_{\mathbb{Z}}\right)\right) \cong \mathbb{Z} \oplus \mathbb{Z}$ e

$$
K_{1}\left(C\left(M_{S L}, \mathcal{K}_{\mathbb{Z}}\right)\right)=\mathbb{Z}\left[u_{1}\right]_{1} \oplus \mathbb{Z}\left[u_{2}\right]_{1}
$$

onde

$$
\begin{gathered}
u_{1}(z, 1)=1+(z-1) E \text { e } u_{2}(z,-1)=1 \\
\text { e } \\
u_{2}(z, 1)=1 \text { e } u_{2}(z,-1)=1+(z-1) E .
\end{gathered}
$$

Pelo isomorfismo $\gamma$ temos que $K_{0}\left(\mathcal{E} / \mathcal{K}_{\mathbb{R}}\right) \cong \mathbb{Z} \oplus \mathbb{Z}$ e $K_{1}\left(\mathcal{E} / \mathcal{K}_{\mathbb{R}}\right) \cong \mathbb{Z} \oplus \mathbb{Z}$.

\section{$3.2 \quad$ A K-teoria $\operatorname{de} \mathcal{A} / \mathcal{K}_{\mathbb{R}}$ e $\mathcal{A}$}

Vamos calcular agora a K-teoria de $\mathcal{A} / \mathcal{K}_{\mathbb{R}}$ e então estaremos prontos para calcular a K-teoria de $\mathcal{A}$. Enunciaremos e demonstraremos antes um Lema que será utilizado no cálculo da aplicação do índice da sequência exata cíclica de seis termos de $\mathcal{A} / \mathcal{K}_{\mathbb{R}}$. Nesta seção e nas que seguem estaremos utilizando as definições de $K_{0}$ e $K_{1}$ de [4], isto é $K_{0}$ é um grupo de diferenças formais de classes de idempotentes (matrizes) de tamanho arbitrário e $K_{1}$ é o grupo de classes de homotopias de matrizes inversíveis de tamanho arbitrário. A demonstração de que as definições apresentadas em [4] e [37] (que usa diferenças formais de classes de projeções ortogonais (matrizes) para $K_{0}$ e de unitários para $K_{1}$ ) são equivalentes encontra-se em [4].

Lema 3.4 Sejam A uma $C^{*}$-álgebra e $J$ um ideal de $A$. Consideremos a sequência exata curta :

$$
0 \longrightarrow J \stackrel{i}{\longrightarrow} A \stackrel{\pi}{\longrightarrow} A / J \longrightarrow 0
$$


onde $i$ é a inclusão e $\pi$ é a projeção canônica.

Se $u \in M_{n}(A / J)$ é inversivel, $\pi(a)=u$ e $\pi(b)=u^{-1}$ então:

$$
\delta_{1}\left([u]_{1}\right)=\left[\left(\begin{array}{cc}
2 a b-(a b)^{2} & a(2-b a)(1-b a) \\
(1-b a) b & (1-b a)^{2}
\end{array}\right)\right]_{0}-\left[\left(\begin{array}{cc}
1 & 0 \\
0 & 0
\end{array}\right)\right]_{0} .
$$

\section{Demonstração:}

Seja $w \in M_{2 n}(A)$ definido por

$$
w=\left(\begin{array}{cc}
2 a-a b a & a b-1 \\
1-b a & b
\end{array}\right)
$$

É fácil verificar que $w$ é inversível e

$$
w^{-1}=\left(\begin{array}{cc}
b & 1-b a \\
a b-1 & 2 a-a b a
\end{array}\right) .
$$

Temos também que

$$
\pi\left(\begin{array}{cc}
2 a-a b a & a b-1 \\
1-b a & b
\end{array}\right)=\left(\begin{array}{cc}
u & 0 \\
0 & u^{-1}
\end{array}\right) .
$$

Pela definição 8.3.1 de [4] segue que

$$
\begin{gathered}
\delta_{1}\left([u]_{1}\right)=\left[\left(\begin{array}{cc}
2 a-a b a & a b-1 \\
1-b a & b
\end{array}\right)\left(\begin{array}{cc}
1 & 0 \\
0 & 0
\end{array}\right)\left(\begin{array}{cc}
b & 1-b a \\
a b-1 & 2 a-a b a
\end{array}\right)\right]_{0}-\left[\left(\begin{array}{ll}
1 & 0 \\
0 & 0
\end{array}\right)\right]_{0} \\
\left.=\left[\begin{array}{cc}
2 a b-(a b)^{2} & a(2-b a)(1-b a) \\
(1-b a) b & (1-b a)^{2}
\end{array}\right)\right]_{0}-\left[\left(\begin{array}{ll}
1 & 0 \\
0 & 0
\end{array}\right)\right]_{0} .
\end{gathered}
$$

Teorema 3.5 Seja $\psi: \mathcal{A} / \mathcal{K}_{\mathbb{R}} \rightarrow \mathcal{A} / \mathcal{E}$ onde $[a]_{\mathcal{K}_{\mathbb{R}}} \mapsto[a]_{\mathcal{E}}, a \in \mathcal{A}$. Temos que $\psi_{*}:$ $K_{0}\left(\mathcal{A} / \mathcal{K}_{\mathbb{R}}\right) \rightarrow K_{0}(\mathcal{A} / \mathcal{E})$ é injetora e a imagem de $\psi_{*}$ é isomorfa a $\mathbb{Z}$. Assim, $K_{0}\left(\mathcal{A} / \mathcal{K}_{\mathbb{R}}\right) \cong$ $\mathbb{Z}$ e temos também que $K_{1}\left(\mathcal{A} / \mathcal{K}_{\mathbb{R}}\right) \cong \mathbb{Z} \oplus \mathbb{Z} \oplus \mathbb{Z}$.

\section{Demonstração:}

Consideremos a sequência exata curta: 


$$
0 \longrightarrow \mathcal{E} / \mathcal{K}_{\mathbb{R}} \stackrel{\varphi}{\longrightarrow} \mathcal{A} / \mathcal{K}_{\mathbb{R}} \stackrel{\psi}{\longrightarrow} \mathcal{A} / \mathcal{A} \longrightarrow 0
$$

onde $\varphi$ é a inclusão e $\psi:[a]_{\mathcal{X}_{\mathbb{R}}} \longmapsto[a]_{\mathcal{E}}, a \in \mathcal{A}$.

$\mathrm{E}$ também sua sequência exata cíclica de seis termos para $\mathrm{K}$-teoria

$$
\begin{array}{cccc}
K_{0}\left(\mathcal{E} / \mathcal{K}_{\mathbb{R}}\right) & \stackrel{\varphi_{*}}{\longrightarrow} K_{0}\left(\mathcal{A} / \mathcal{K}_{\mathbb{R}}\right) \stackrel{\psi_{*}}{\longrightarrow} & K_{0}(\mathcal{A} / \mathcal{E}) \\
\uparrow \delta_{1} & & & \mid \delta_{0} \\
K_{1}(\mathcal{A} / \mathcal{E}) & \stackrel{\psi_{*}}{\longleftarrow} K_{1}\left(\mathcal{A} / \mathcal{K}_{\mathbb{R}}\right) \stackrel{\varphi_{*}}{\longleftarrow} K_{1}\left(\mathcal{E} / \mathcal{K}_{\mathbb{R}}\right)
\end{array}
$$

Substituindo as K-teorias que conhecemos temos

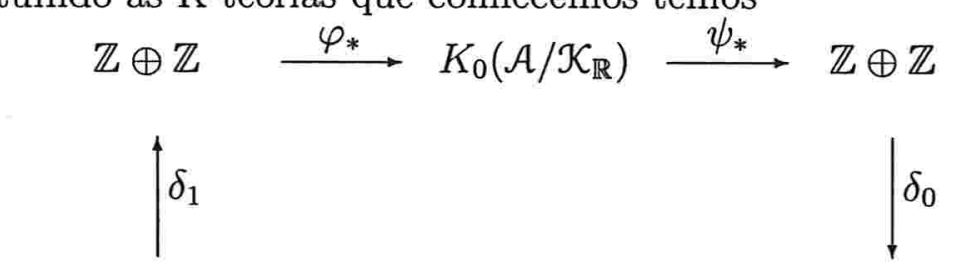

$$
\mathbb{Z} \oplus \mathbb{Z} \oplus \mathbb{Z} \oplus \mathbb{Z} \stackrel{\psi_{*}}{\longleftarrow} K_{1}\left(\mathcal{A} / \mathcal{K}_{\mathbb{R}}\right) \stackrel{\varphi_{*}}{\longleftarrow} \mathbb{Z} \oplus \mathbb{Z}
$$

Precisamos calcular $\delta_{0}$ e $\delta_{1}$ para conhecermos então a K-teoria de $\mathcal{A} / \mathcal{K}_{\mathbb{R}}$.

Vamos primeiro calcular $\delta_{0}\left(\left[[b(D)]_{\varepsilon}\right]_{0}\right)$, onde $\left[[b(D)]_{\varepsilon}\right]_{0}$ foi descrito na página 55 equação (3.1). Pela Proposição 12.2.2 de [37] sabemos que precisamos de um elemento $x \in \mathcal{A}$ tal que $[x]_{\mathcal{K}_{\mathrm{R}}}$ seja auto-adjunto e $\psi\left([x]_{\mathcal{K}_{\mathbb{R}}}\right)=[b(D)]_{\mathcal{E}}$.

Como $b(D)$ já é auto-adjunto em $\mathcal{A}$ basta então tomarmos $x=b(D)$. Pela Proposição 12.2 .2 de [37] temos que existe um único elemento $u \in U\left(\widetilde{\mathcal{E} / \mathcal{K}_{\mathbb{R}}}\right)$ tal que $\bar{\varphi}(u)=e^{2 \pi i[b(D)]_{\boldsymbol{x}_{\mathbb{R}}}}$. Como $\widetilde{\varepsilon / \mathcal{K}_{\mathbb{R}}} \subset \mathcal{A} / \mathcal{K}_{\mathbb{R}}$ temos $\varphi=\bar{\varphi}$, logo pela definição de $\varphi$ temos que $u=e^{2 \pi i[b(D)] \boldsymbol{x}_{\mathbb{R}}}$. Portanto o gerador $\left[[b(D)]_{\varepsilon}\right]_{0}$ de $K_{0}(\mathcal{A} / \mathcal{E})$ é levado em $\left.\left.-\left[e^{2 \pi i[b(D)}\right]\right]_{\mathbb{R}}\right]_{1} \in K_{1}\left(\mathcal{E} / \mathcal{K}_{\mathbb{R}}\right)$ pela aplicação $\delta_{0}$. Utilizando o isomorfismo $\gamma$ da Proposição 1.24 e homotopias verificamos em seguida que $\left[e^{2 \pi i[b(D)] x_{\mathrm{R}}}\right]_{1}$ corresponde ao elemento $(-1,-1)$ de $K_{1}\left(C\left(M_{S L}, \mathcal{K}_{\mathbb{Z}}\right)\right)$.

Usando o isomorfismo da Proposição 1.24 temos que $\left.\left.\left[e^{2 \pi i[b(D)}\right]\right]_{\mathbb{R}}\right]_{1}$ é levado em $[h]_{1}$ onde $h$ é a função:

$$
\begin{aligned}
& h: M_{S L} \longrightarrow \mathcal{K}_{\mathbb{Z}} \\
& \left(e^{2 \pi i \varphi}, \pm 1\right) \longmapsto Y_{\varphi}\left(e^{2 \pi i b(M-\varphi)}\right) Y_{-\varphi} .
\end{aligned}
$$


Com respeito ao isomorfismo da Proposição 3.3, sabemos que o elemento que corresponde a $(1,1) \in \mathbb{Z} \oplus \mathbb{Z}$ em $K_{1}\left(C\left(M_{S L}, \mathcal{K}_{\mathbb{Z}}\right)\right)$ é dado pela classe em $K_{1}$ de

$$
\begin{array}{ccc}
l: M_{S L} & \longrightarrow & \mathcal{K}_{\mathbb{Z}} \\
(z, \pm 1) & \longmapsto 1+(z-1) E,
\end{array}
$$

onde $E\left(\left(a_{n}\right)_{n}\right)=a_{0} e_{0}$. Seja $a \in C S(\mathbb{R})$ então,

$$
Y_{\varphi+1} a(M-(\varphi+1)) Y_{-(\varphi+1)}=Y_{\varphi} Y_{1} a(M-(\varphi+1)) Y_{-1} Y_{-\varphi}=Y_{\varphi} a(M-\varphi) Y_{-\varphi}
$$

$\operatorname{logo}_{\varphi}\left(e^{2 \pi i b(M-\varphi)}\right) Y_{-\varphi}$ é periódica de período 1. Consideremos então $Y_{\varphi}\left(e^{2 \pi i b(M-\varphi)}\right) Y_{-\varphi}$ para $\varphi \in\left[-\frac{1}{2}, \frac{1}{2}\right]$.

Sem perda de generalidade podemos supor que a função $b$ é constante em $(-\infty,-1 / 5]$ e em $[1 / 5,+\infty)$. Se $1 / 5 \leq|\varphi| \leq 1 / 2$ então $|j-\varphi| \geq \frac{1}{5}$ para todo $j$. Se $|\varphi|<1 / 5$ então $|j-\varphi| \geq \frac{1}{5}$ para todo $j$ não nulo.

Logo para todo $\varphi \in[-1 / 2,1 / 2]$ temos que

$$
e^{2 \pi i b(j-\varphi)}= \begin{cases}e^{2 \pi i b(-\varphi)} & \text { se } j=0 \\ 1 & \text { se } j \neq 0\end{cases}
$$

Portanto $e^{2 \pi i b(M-\varphi)}=I d+\left(e^{2 \pi i b(-\varphi)}-I d\right) E$ para todo $|\varphi| \leq 1 / 2$. Seja $g(\varphi)=e^{2 \pi i b(-\varphi)}-$ $I d$ temos que $g \in C_{c}^{\infty}\left(\left[-\frac{1}{5}, \frac{1}{5}\right]\right)$ e $e^{2 \pi i b(M-\varphi)}=g(\varphi) E+I d \operatorname{logo} Y_{\varphi}\left(e^{2 \pi i b(M-\varphi)}\right) Y_{-\varphi}=$ $Y_{\varphi}(g(\varphi) E+I d) Y_{-\varphi}$, para todo $|\varphi| \leq 1 / 2$.

Seja $Z_{t}=Y_{h(t)}$ onde $h:[-1 / 2,1 / 2] \rightarrow \mathbb{R} \operatorname{com} h \equiv 0$ em $[-1 / 4,1 / 4]$. Logo $Z_{t}=I d$ se $t \in\left[-\frac{1}{4}, \frac{1}{4}\right]$.

Seja $\left.v:[0,1] \longrightarrow \mathcal{U}\left(C([-1 / 2,1 / 2]), \mathcal{K}_{\mathbb{Z}}\right)\right)$ onde $x \longmapsto v_{x}$ definida por $v_{x}(t)=$ $Y_{(1-x) t+x h(t)}(g(t) E+1) Y_{-[(1-x) t+x h(t)]}$. Se $|t| \geq 1 / 5$ temos $g(t) E+I d=I d$. Logo para todo $x, v_{x}$ pode ser encarado como um elemento de $\mathcal{U}\left(C\left(S^{1}, \mathcal{K}_{\mathbb{Z}}\right)\right)$ e $v_{0}(t)=Y_{t}(g(t) E+1) Y_{-t}$ e $v_{1}(t)=Y_{h(t)}(g(t) E+1) Y_{-h(t)}=Z_{t}(g(t) E+1) Z_{-t}$. Portanto $Y_{t}(g(t) E+1) Y_{-t}$ e $Z_{t}(g(t) E+1) Z_{-t}$ são elementos homotópicos de $C\left(S^{1}, \mathcal{K}_{\mathbb{Z}}\right)$.

Como $Z_{t}=I d$ se $t \in\left[-\frac{1}{4}, \frac{1}{4}\right]$ e $g$ tem suporte contido em $\left[-\frac{1}{5}, \frac{1}{5}\right]$ temos que $Z_{t}(g(t) E+$ 1) $Z_{-t}=g(t) E+1$ para todo $t \in[-1 / 2,1 / 2]$. Então pela Proposição 8.1 .4 de [37] e pelos resultados acima temos

$$
\left[Y_{t}(g(t) E+1) Y_{-t}\right]_{1}=\left[Z_{t}(g(t) E+1) Z_{-t}\right]_{1}=[g(t) E+1]_{1}
$$


onde $F(t)$ denota a função $\left(e^{2 \pi i t}, \pm 1\right) \ni M_{S L} \mapsto F(t) \in \mathcal{K}_{\mathbb{Z}}$.

Usando o que foi visto na página 47 sobre número de rotação temos que as funções $\bar{z}=(z \longmapsto \bar{z})$ e $\left(e^{2 \pi i \varphi} \longmapsto e^{2 \pi i b(-\varphi)}\right)=\tilde{g}(\varphi)$ são homotópicas em $\mathcal{U}\left(C\left(S^{1}\right)\right)$. Seja então $l:[0,1] \longrightarrow \mathcal{U}\left(C\left(S^{1}\right)\right)$ tal que $l(0)=\bar{z}$ e $l(1)=\tilde{g}(\varphi)$ e seja $v:[0,1] \longrightarrow \mathcal{U}\left(C\left(S^{1}, \mathcal{K}_{\mathbb{Z}}\right)\right)$ dada por $x \longmapsto(l(x)-1) E+1$. Temos que $v(0)=(\tilde{g}(\varphi)-1) E+1=g(\varphi) E+1$ e $v(1)=(\bar{z}-1) E+1$. E sabemos que $(\bar{z}-1) E+1$ é o -1 em $K_{1}\left(C\left(S^{1}, \mathcal{K}_{\mathbb{Z}}\right)\right)$.(veja demonstração da Proposição 3.3)

Portanto pela Proposição 8.1.4 de [37] temos $\left.[g(\varphi) E+1]_{1}=[(\bar{z}-1) E+1)\right]_{1}$, logo $\delta_{0}((1,0))=(1,1)$

Com uma demonstração análoga podemos mostrar que $\delta_{0}((0,1))=(-1,-1)$. Então temos que $\delta_{0}((x, y))=(x-y)(1,1)$. Com isto segue que $\operatorname{Ker} \delta_{0}=\{(x, y) \in \mathbb{Z} \oplus \mathbb{Z}: x=$ $y\} \cong \mathbb{Z}$ e Im $\delta_{0}=\{(a, a): a \in \mathbb{Z}\} \cong \mathbb{Z}$.

Com isso encerramos o cálculo de $\delta_{0}$ e passaremos agora a calcular a aplicação $\delta_{1}$.

Já sabemos pela Proposição 3.2 que os quatro geradores de $K_{1}(\mathcal{A} / \mathcal{E})$ são $\left[\left[A_{1}\right]_{\mathcal{E}}\right]_{1}$, $\left[\left[A_{2}\right]_{\mathcal{E}}\right]_{1},\left[\left[A_{3}\right]_{\mathcal{E}}\right]_{1},\left[\left[A_{4}\right]_{\mathcal{E}}\right]_{1}$, onde $A_{1}=L(M) b(D)+c(D), A_{2}=\tilde{L}(M) b(D)+c(D), A_{3}=$ $b(D)+L(M) c(D), A_{4}=b(D)+\tilde{L}(M) c(D)$.

É obvio que $a=\left[A_{1}\right]_{\mathcal{K}_{\mathbb{R}}}$ é uma pré-imagem de $u=\left[A_{1}\right]_{\varepsilon}$. Como $\left[A_{1}\right]_{\varepsilon}=u$ é unitário temos que $b=a^{*}$ é uma pré-imagem de $u^{-1}$. Pelo Lema 3.4 temos que

$$
\delta_{1}\left(\left[\left[A_{1}\right]_{\mathcal{E}}\right]_{1}\right)=\left[\left(\begin{array}{cc}
2 a a^{*}-\left(a a^{*}\right)^{2} & a\left(2-a^{*} a\right)\left(1-a^{*} a\right) \\
\left(1-a^{*} a\right) a^{*} & \left(1-a^{*} a\right)^{2}
\end{array}\right)\right]_{0}-\left[\left(\begin{array}{ll}
1 & 0 \\
0 & 0
\end{array}\right)\right]_{0}
$$

onde $\delta_{1}\left(\left[\left[A_{1}\right]_{\varepsilon}\right]_{1}\right) \in K_{0}\left(\mathcal{E} / \mathcal{K}_{\mathbb{R}}\right) \stackrel{\gamma_{*}}{\cong} K_{0}\left(C\left(M_{S L}, \mathcal{K}_{\mathbb{Z}}\right)\right) \stackrel{\nu_{*}}{\cong} K_{0}\left(\mathcal{K}_{\mathbb{Z}}\right) \oplus K_{0}\left(\mathcal{K}_{\mathbb{Z}}\right)$ (veja Proposição 3.3). Seja $\eta=\nu_{*} \circ \gamma_{*}$ então considerando $\alpha=\gamma_{A_{1}}(1,1), \alpha^{\prime}=\gamma_{A_{1}}(1,-1), \beta=\gamma_{A_{1}^{*}}(1,1) \mathrm{e}$ $\beta^{\prime}=\gamma_{A_{i}^{*}}(1,-1)$ temos

$$
\begin{gathered}
\eta \circ \delta_{1}\left(\left[\left[A_{1}\right]_{\varepsilon}\right]_{1}\right)=\left(\left[\begin{array}{cc}
2 \alpha \beta-(\alpha \beta)^{2} & \alpha(2-\beta \alpha)(1-\beta \alpha) \\
(1-\beta \alpha) \beta & (1-\beta \alpha)^{2}
\end{array}\right)\right]_{0}-\left[\left(\begin{array}{ll}
1 & 0 \\
0 & 0
\end{array}\right)\right]_{0}, \\
\left.\left[\left(\begin{array}{cc}
2 \alpha^{\prime} \beta^{\prime}-\left(\alpha^{\prime} \beta^{\prime}\right)^{2} & \alpha^{\prime}\left(2-\beta^{\prime} \alpha^{\prime}\right)\left(1-\beta^{\prime} \alpha^{\prime}\right) \\
\left(1-\beta^{\prime} \alpha^{\prime}\right) \beta^{\prime} & \left(1-\beta^{\prime} \alpha^{\prime}\right)^{2}
\end{array}\right)\right]_{0}-\left[\left(\begin{array}{ll}
1 & 0 \\
0 & 0
\end{array}\right)\right]_{0}\right)
\end{gathered}
$$

Vamos observar que $\gamma_{A_{1}}(1, \pm 1) \notin \mathcal{K}_{\mathbb{Z}}$, já que $A_{1} \in \mathcal{A}$ e $A_{1} \notin \mathcal{E}$. Observemos também que $A_{1}^{*} A_{1}-I d \in \mathcal{E}$ então $\gamma_{A_{1}^{*} A_{1}}(1, \pm 1)-\gamma_{I d}(1, \pm 1) \in \mathcal{K}_{\mathbb{Z}}$. Portanto $\left[\gamma_{A_{1}}(1,1)\right]_{\mathcal{K}_{\mathrm{Z}}}$ e 
$\left[\gamma_{A_{1}}(1,-1)\right]_{\mathcal{K}_{\mathbb{Z}}}$ são inversíveis em $\mathcal{L}\left(L^{2}(\mathbb{Z})\right) / \mathcal{K}_{\mathbb{Z}}$.

Temos então pelo Lema 3.4 que

$$
\bar{\delta}_{1}\left(\left[\gamma_{A_{1}}(1,1)\right]_{1}\right)=\left[\left(\begin{array}{cc}
2 \alpha \beta-(\alpha \beta)^{2} & \alpha(2-\beta \alpha)(1-\beta \alpha) \\
(1-\beta \alpha) \beta & (1-\beta \alpha)^{2}
\end{array}\right)\right]_{0}-\left[\left(\begin{array}{ll}
1 & 0 \\
0 & 0
\end{array}\right)\right]_{0}
$$

e

$$
\bar{\delta}_{1}\left(\left[\gamma_{A_{1}}(1,-1)\right]_{1}\right)=\left[\left(\begin{array}{cc}
2 \alpha^{\prime} \beta^{\prime}-\left(\alpha^{\prime} \beta^{\prime}\right)^{2} & \alpha^{\prime}\left(2-\beta^{\prime} \alpha^{\prime}\right)\left(1-\beta^{\prime} \alpha^{\prime}\right) \\
\left(1-\beta^{\prime} \alpha^{\prime}\right) \beta^{\prime} & \left(1-\beta^{\prime} \alpha^{\prime}\right)^{2}
\end{array}\right)\right]_{0}-\left[\left(\begin{array}{ll}
1 & 0 \\
0 & 0
\end{array}\right)\right]_{0}
$$

onde $\bar{\delta}_{1}$ é o índice da sequência de K-teoria associada a sequência exata curta abaixo

$$
0 \longrightarrow \mathcal{K}_{\mathbb{Z}} \stackrel{i}{\longrightarrow} \mathcal{L}\left(L^{2}(\mathbb{Z})\right) \stackrel{\pi}{\longrightarrow} \mathcal{L}\left(L^{2}(\mathbb{Z})\right) / \mathcal{K}_{\mathbb{Z}} \longrightarrow 0
$$

Mas o índice para esta sequência é o índice de Fredholm por 9.4.2 de [37] . Por 19.1.14 de [18] temos que

$$
\text { ind } \gamma_{A_{1}}(1, \pm 1)=\operatorname{Tr}\left(\left(I d-\gamma_{A_{1}^{*}}(1, \pm 1) \gamma_{A_{1}}(1, \pm 1)\right)^{N}\right)-\operatorname{Tr}\left(\left(I d-\gamma_{A_{1}}(1, \pm 1) \gamma_{A_{1}^{*}}(1, \pm 1)\right)^{N}\right)
$$

para algum $N>0$ natural para o qual se tenha

$$
\left(I d-\gamma_{A_{\mathbf{1}}^{*}}(1, \pm 1) \gamma_{A_{1}}(1, \pm 1)\right)^{N} \text { e }\left(I d-\gamma_{A_{1}}(1, \pm 1) \gamma_{A_{1}^{*}}(1, \pm 1)\right)^{N} \text { trace-class. }
$$

O mesmo raciocínio vale para $A_{2}, A_{3}, A_{4}$.

Utilizando a Proposição 1.25 temos que

$$
\begin{aligned}
& \gamma_{A_{1}}(1,-1)=I d \text { e } \gamma_{A_{1}^{*}}(1,-1)=I d, \\
& \gamma_{A_{1}}(1,1)=b(M-1) Y_{-1}+Y_{1} c(M-1) Y_{-1} \text { e } \gamma_{A_{1}^{*}}(1,1)=Y_{1} b(M-1)+Y_{1} c(M-1) Y_{-1}, \\
& \gamma_{A_{2}}(1,-1)=b(M-1) Y_{-1}+Y_{1} c(M-1) Y_{-1} \text { e } \gamma_{A_{2}^{*}}(1,-1)=Y_{1} b(M-1)+Y_{1} c(M-1) Y_{-1}, \\
& \gamma_{A_{2}}(1,1)=I d \text { e } \gamma_{A_{2}^{*}}(1,1)=I d, \\
& \gamma_{A_{3}}(1,-1)=I d \text { e } \gamma_{A_{3}^{*}}(1,-1)=I d, \\
& \gamma_{A_{3}}(1,1)=Y_{1} b(M-1) Y_{-1}+c(M-1) Y_{-1} \text { e } \gamma_{A_{3}^{*}}(1,1)=Y_{1} b(M-1) Y_{-1}+Y_{1} c(M-1), \\
& \gamma_{A_{4}}(1,-1)=Y_{1} b(M-1) Y_{-1}+c(M-1) Y_{-1} \text { e } \gamma_{A_{4}^{*}}(1,-1)=Y_{1} b(M-1) Y_{-1}+Y_{1} c(M-1), \\
& \gamma_{A_{4}}(1,1)=I d \text { e } \gamma_{A_{4}^{*}}(1,1)=I d .
\end{aligned}
$$

Construindo as matrizes desses operadores podemos verificar que para $N=1$ temos que $\left(I d-\gamma_{A_{i}^{*}}(1, \pm 1) \gamma_{A_{i}}(1, \pm 1)\right)$ e $\left(I d-\gamma_{A_{i}}(1, \pm 1) \gamma_{A_{i}^{*}}(1, \pm 1)\right)$, para $i=1, \ldots, 4$, são de posto finito. 
Apresentamos a seguir as matrizes dos operadores $\left(I d-\gamma_{A_{1}^{*}}(1,1) \gamma_{A_{1}}(1,1)\right)$ e $\left(I d-\gamma_{A_{1}}(1,1) \gamma_{A_{1}^{*}}(1,1)\right)$.

$$
\left[\begin{array}{ccccccccccc}
0 & 0 & \ldots & \ldots & \ldots & 0 & 0 & 0 & 0 & 0 & \ldots \\
0 & \ddots & \vdots & \vdots & \vdots & \vdots & \vdots & \vdots & \vdots & \vdots & \vdots \\
\vdots & \vdots & \ddots & \vdots & \vdots & \vdots & \vdots & \vdots & \vdots & \vdots & \vdots \\
\vdots & \vdots & \vdots & \ddots & \vdots & \vdots & \vdots & \vdots & \vdots & \vdots & \vdots \\
\vdots & \vdots & \vdots & \vdots & \ddots & \vdots & \vdots & \vdots & \vdots & \vdots & \vdots \\
\vdots & \vdots & \vdots & \vdots & \vdots & 0 & \vdots & \vdots & \vdots & \vdots & \vdots \\
\vdots & \vdots & \vdots & \vdots & \vdots & \vdots & 0 & -1 / 2 & 0 & \vdots & \vdots \\
\vdots & \vdots & \vdots & \vdots & \vdots & \vdots & -1 / 2 & 1 / 2 & -1 / 2 & 0 & \vdots \\
\vdots & \vdots & \vdots & \vdots & \vdots & \vdots & 0 & -1 / 2 & 0 & \vdots & \vdots \\
\vdots & \vdots & \vdots & \vdots & \vdots & \vdots & \vdots & 0 & 0 & \ddots & \vdots \\
\vdots & \vdots & \vdots & \vdots & \vdots & \vdots & \vdots & \vdots & \vdots & \vdots & \ddots
\end{array}\right]
$$

$$
\left[\begin{array}{ccccccccccc}
0 & 0 & \ldots & \ldots & \ldots & 0 & 0 & 0 & 0 & 0 & \ldots \\
0 & \ddots & \vdots & \vdots & \vdots & \vdots & \vdots & \vdots & \vdots & \vdots & \vdots \\
\vdots & \vdots & \ddots & \vdots & \vdots & \vdots & \vdots & \vdots & \vdots & \vdots & \vdots \\
\vdots & \vdots & \vdots & \ddots & \vdots & \vdots & \vdots & \vdots & \vdots & \vdots & \vdots \\
\vdots & \vdots & \vdots & \vdots & \ddots & \vdots & \vdots & \vdots & \vdots & \vdots & \vdots \\
\vdots & \vdots & \vdots & \vdots & \vdots & 0 & \vdots & \vdots & \vdots & \vdots & \vdots \\
\vdots & \vdots & \vdots & \vdots & \vdots & \vdots & -1 / 4 & -1 / 4 & 0 & \vdots & \vdots \\
\vdots & \vdots & \vdots & \vdots & \vdots & \vdots & -1 / 4 & -1 / 4 & 0 & 0 & \vdots \\
\vdots & \vdots & \vdots & \vdots & \vdots & \vdots & 0 & 0 & 0 & \vdots & \vdots \\
\vdots & \vdots & \vdots & \vdots & \vdots & \vdots & \vdots & 0 & 0 & \ddots & \vdots \\
\vdots & \vdots & \vdots & \vdots & \vdots & \vdots & \vdots & \vdots & \vdots & \vdots & \ddots
\end{array}\right]
$$

Obtemos então:

$$
\begin{aligned}
& \bar{\delta}_{1}\left(\gamma_{A_{1}}(1,1)\right)=\operatorname{Tr}\left(I d-\gamma_{A_{1}^{*}}(1,1) \gamma_{A_{1}}(1,1)\right)-\operatorname{Tr}\left(I d-\gamma_{A_{1}}(1,1) \gamma_{A_{1}^{*}}(1,1)\right)=1, \\
& \bar{\delta}_{1}\left(\gamma_{A_{1}}(1,-1)\right)=\operatorname{Tr}\left(I d-\gamma_{A_{1}^{*}}(1,-1) \gamma_{A_{1}}(1,-1)\right)-\operatorname{Tr}\left(I d-\gamma_{A_{1}}(1,-1) \gamma_{A_{1}^{*}}(1,-1)\right)=0, \\
& \bar{\delta}_{1}\left(\gamma_{A_{2}}(1,1)\right)=\operatorname{Tr}\left(I d-\gamma_{A_{2}^{*}}(1,1) \gamma_{A_{2}}(1,1)\right)-\operatorname{Tr}\left(I d-\gamma_{A_{2}}(1,1) \gamma_{A_{2}^{*}}(1,1)\right)=0, \\
& \bar{\delta}_{1}\left(\gamma_{A_{2}}(1,-1)\right)=\operatorname{Tr}\left(I d-\gamma_{A_{2}^{*}}(1,-1) \gamma_{A_{2}}(1,-1)\right)-\operatorname{Tr}\left(I d-\gamma_{A_{2}}(1,-1) \gamma_{A_{2}^{*}}(1,-1)\right)=1, \\
& \bar{\delta}_{1}\left(\gamma_{A_{3}}(1,1)\right)=\operatorname{Tr}\left(I d-\gamma_{A_{3}^{*}}(1,1) \gamma_{A_{3}}(1,1)\right)-\operatorname{Tr}\left(I d-\gamma_{A_{3}}(1,1) \gamma_{A_{3}^{*}}(1,1)\right)=-1,
\end{aligned}
$$


$\bar{\delta}_{1}\left(\gamma_{A_{3}}(1,-1)\right)=\operatorname{Tr}\left(I d-\gamma_{A_{3}^{*}}(1,-1) \gamma_{A_{3}}(1,-1)\right)-\operatorname{Tr}\left(I d-\gamma_{A_{3}}(1,-1) \gamma_{A_{3}^{*}}(1,-1)\right)=0$,

$\bar{\delta}_{1}\left(\gamma_{A_{4}}(1,1)\right)=\operatorname{Tr}\left(I d-\gamma_{A_{4}^{*}}(1,1) \gamma_{A_{4}}(1,1)\right)-\operatorname{Tr}\left(I d-\gamma_{A_{4}}(1,1) \gamma_{A_{4}^{*}}(1,1)\right)=0$,

$\bar{\delta}_{1}\left(\gamma_{A_{4}}(1,-1)\right)=\operatorname{Tr}\left(I d-\gamma_{A_{4}^{*}}(1,-1) \gamma_{A_{4}}(1,-1)\right)-\operatorname{Tr}\left(I d-\gamma_{A_{4}}(1,-1) \gamma_{A_{4}^{*}}(1,-1)\right)=-1$.

Portanto podemos concluir que

$$
\begin{gathered}
\eta \circ \delta_{1}\left(\left[\left[A_{1}\right]_{\varepsilon}\right]_{1}\right)=(1,0), \\
\eta \circ \delta_{1}\left(\left[\left[A_{2}\right]_{\varepsilon}\right]_{1}\right)=(0,1), \\
\eta \circ \delta_{1}\left(\left[\left[A_{3}\right]_{\varepsilon}\right]_{1}\right)=(-1,0), \\
\eta \circ \delta_{1}\left(\left[\left[A_{4}\right]_{\varepsilon}\right]_{1}\right)=(0,-1) .
\end{gathered}
$$

Segue da exatidão da sequência exata cíclica de seis termos da página 59 que $\operatorname{Im} \delta_{1}=$ $\operatorname{Ker} \varphi_{*} \cong \mathbb{Z} \oplus \mathbb{Z} ; \operatorname{Im} \varphi_{*}=\operatorname{Ker} \psi_{*}=0$ e $\operatorname{Im} \psi_{*}=\operatorname{Ker} \delta_{0}=\mathbb{Z}(1,1)$ onde $(1,1) \in K_{0}(\mathcal{A} / \mathcal{E})=$ $\mathbb{Z}\left[[b(D)]_{\mathcal{E}}\right]_{0} \oplus \mathbb{Z}\left[[c(D)]_{\mathcal{E}}\right]_{0}$ (veja página 55 , (3.1)). Portanto

$$
K_{0}\left(\mathcal{A} / \mathcal{K}_{\mathbb{R}}\right)=\mathbb{Z}\left[[I d]_{\mathcal{K}_{\mathbb{R}}}\right]_{0}
$$

Pelos cálculos acima temos que $\operatorname{Ker} \delta_{1}=\{(x, y, z, w) \in \mathbb{Z} \oplus \mathbb{Z} \oplus \mathbb{Z} \oplus \mathbb{Z}: y=w \quad$ e $\quad x=$ $z\}$ logo $(1,0,1,0)$ e $(0,1,0,1) \in K_{1}(\mathcal{A} / \mathcal{E})=\mathbb{Z}\left[\left[A_{1}\right]_{\mathcal{E}}\right]_{1} \oplus \mathbb{Z}\left[\left[A_{2}\right]_{\mathcal{E}}\right]_{1} \oplus \mathbb{Z}\left[\left[A_{3}\right]_{\mathcal{E}}\right]_{1} \oplus \mathbb{Z}\left[\left[A_{4}\right]_{\mathcal{E}}\right]_{1}$ (veja página $56,(3.2)$ ) geram $\operatorname{Ker} \delta_{1}$.

Novamente pela exatidão da sequência exata cíclica de seis termos temos que $\operatorname{Im} \psi_{*}=$ $\operatorname{Ker} \delta_{1} \cong \mathbb{Z} \oplus \mathbb{Z}$. Pelo Teorema do homomorfismo e exatidão da sequência exata cíclica temos que $\operatorname{Ker} \psi_{*}=\operatorname{Im} \varphi_{*} \cong \mathbb{Z} \oplus \mathbb{Z} / \mathbb{Z}(1,1) \cong \mathbb{Z}(1,0)$ onde $(1,0) \in K_{1}\left(\mathcal{E} / \mathcal{K}_{\mathbb{R}}\right)$.

Podemos então construir a seguinte sequência exata curta

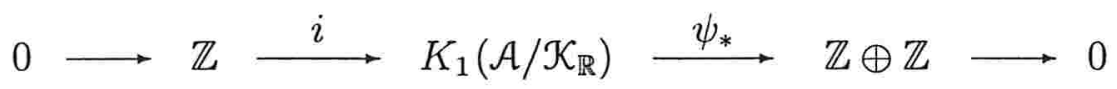

onde $i$ é a inclusão.

Pela Proposição 1.25 e demonstração do Teorema 3.5 temos que $\left[b(M) e^{2 \pi i c(D)}+\right.$ $c(M)]_{\mathcal{K}_{\mathbb{R}}}$ b e $c$ como na página 54 , corresponde ao $(1,0)$ de $K_{1}\left(\mathcal{E} / \mathcal{K}_{\mathbb{R}}\right)$. Então usando a sequência exata acima temos que

$$
K_{1}\left(\mathcal{A} / \mathcal{K}_{\mathbb{R}}\right)=\mathbb{Z}\left[[L(M)]_{\mathcal{K}_{\mathbb{R}}}\right]_{1} \oplus \mathbb{Z}\left[[\tilde{L}(M)]_{\mathcal{K}_{\mathbb{R}}}\right]_{1} \oplus \mathbb{Z}\left[\left[b(M) e^{2 \pi i c(D)}+c(M)\right]_{\mathcal{K}_{\mathbb{R}}}\right]_{1}
$$

Agora já estamos prontos para calcular a K-teoria da $C^{*}$ - álgebra $\mathcal{A}$. 
Teorema 3.6 Seja $\pi: \mathcal{A} \longrightarrow \mathcal{A} / \mathcal{K}_{\mathbb{R}}$ a projeção canônica. Então $\pi_{*}: K_{i}(\mathcal{A}) \longrightarrow \operatorname{Im} \pi_{*}$ é um isomorfismo. Logo $K_{0}(\mathcal{A}) \cong \mathbb{Z}$ e $K_{1}(\mathcal{A}) \cong \mathbb{Z} \oplus \mathbb{Z}$.

\section{Demonstração:}

Consideremos a sequência exata curta

$$
0 \longrightarrow \mathcal{K}_{\mathbb{R}} \stackrel{i}{\longrightarrow} \mathcal{A} \stackrel{\pi}{\longrightarrow} \mathcal{A} / \mathcal{K}_{\mathbb{R}} \longrightarrow 0
$$

e sua sequência exata ciclíca de seis termos para K-teoria

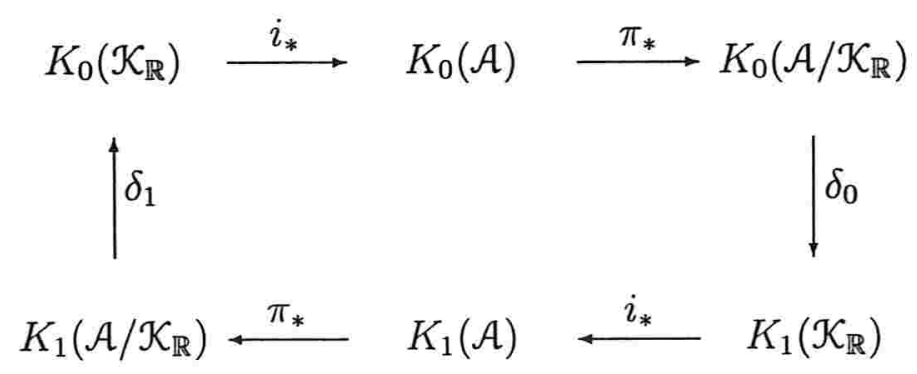

Substituindo as K-teorias conhecidas temos

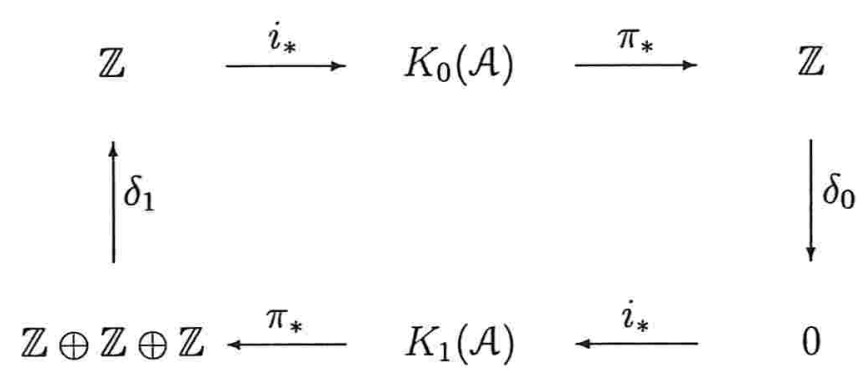

Sabemos pela Proposição 9.4.4 de [37] que o índice para esta sequência é o índice de Fredholm. Vamos calcular $\delta_{1}$ nos geradores de $K_{1}\left(\mathcal{A} / \mathcal{K}_{\mathbb{R}}\right)$.

Temos que $b(M) e^{2 \pi i c(D)}+c(M) \in \mathcal{C}$, logo $\left[b(M) e^{2 \pi i c(D)}+c(M)\right]_{\mathcal{K}_{\mathbb{R}}} \in \mathcal{C} / \mathcal{K}_{\mathbb{R}}$ e como foi visto no Capítulo 2, Teorema $2.13, \delta_{1}\left(\left[[T]_{\mathcal{K}_{\mathbb{R}}}\right]_{1}\right)=i n d(T)=w\left(\sigma_{T}\right)$ nesta álgebra.

Logo $\delta_{1}\left(\left[\left[b(M) e^{2 \pi i c(D)}+c(M)\right]_{\mathcal{K}_{\mathbb{R}}}\right]_{1}\right)=w\left(\sigma_{b(M) e^{2 \pi i c(D)}+c(M)}\right)=-1$. Sabemos também que $\operatorname{ind}(L(M))=\operatorname{ind}(\tilde{L}(M))=0$, pois são operadores de multiplicação. Portanto $\delta_{1}$ é sobrejetora e $\delta_{1}(x, y, z)=-z$.

Pela exatidão da sequência exata cíclica de seis termos temos que $\operatorname{Im} i_{*}=\operatorname{Ker} \pi_{*}=0$; $\operatorname{Im} \pi_{*}=\operatorname{Ker} \delta_{0}=\mathbb{Z} ; \operatorname{Im} i_{*}=\operatorname{Ker} \pi_{*}=0$ e $\operatorname{Im} \pi_{*}=\operatorname{Ker} \delta_{1} \cong \mathbb{Z} \oplus \mathbb{Z}, \log \mathrm{o}$

$$
K_{0}(\mathcal{A})=\mathbb{Z}[I d]_{0} \quad \text { e } \quad K_{1}(\mathcal{A})=\mathbb{Z}[L(M)]_{1} \oplus \mathbb{Z}[\tilde{L}(M)]_{1} .
$$




\section{Capítulo 4}

\section{K-teoria de $\mathcal{A}$ por produto cruzado}

Neste capítulo calcularemos, novamente, a K-teoria de $\mathcal{A}$ utilizando a seguinte estratégia. Primeiro provaremos que a $\operatorname{Im} \gamma$, onde $\gamma$ é a aplicação da Proposição 1.25 tem estrutura de produto cruzado e então usando a sequência exata de Pimsner-Voiculescu [34] calcularemos sua K-teoria. Utilizando então a sequência exata curta induzida por $\gamma$

$$
0 \longrightarrow J_{0} / \mathcal{K}_{\mathbb{R}} \longrightarrow \mathcal{A} / \mathcal{K}_{\mathbb{R}} \longrightarrow \operatorname{Im} \gamma \longrightarrow 0
$$

calculamos a K-teoria de $\mathcal{A} / \mathcal{K}_{\mathbb{R}}$. De posse desta K-teoria e usando a sequência exata curta abaixo calculamos a K-teoria de $\mathcal{A}$.

$$
0 \longrightarrow \mathcal{K}_{\mathbb{R}} \longrightarrow \mathcal{A} \longrightarrow \mathcal{A} / \mathcal{K}_{\mathbb{R}} \longrightarrow 0
$$

O que diferencia a estratégia deste capítulo para o anterior é que no Capítulo 3 utilizamos uma sequência exata curta induzida pelo $\sigma$-símbolo para calcular a K-teoria de $\mathcal{A} / \mathcal{K}_{\mathbb{R}}$.

Um estudo geral sobre produto cruzado de $C^{*}$ - álgebras pode ser encontrado no Capítulo 7 de [33]. Aqui, trabalharemos apenas em casos onde a $C^{*}$ - álgebra $A$ tem unidade e o grupo é $\mathbb{Z}$ com a topologia discreta.

Dados uma $C^{*}$ - álgebra $A$ com unidade, o grupo $\mathbb{Z}$ com a topologia discreta, e uma ação $\alpha$ de $\mathbb{Z}$ em $A$, define-se uma nova $C^{*}$ - álgebra $A \underset{\alpha}{\rtimes} \mathbb{Z}$ como sendo a $C^{*}$ - álgebra envolvente de $l_{1}(\mathbb{Z}, A)$ (seção (2.7) de [14]), esta vista como uma álgebra de Banach com a involução, o produto e a norma definidos respectivamente por: 


$$
\begin{gathered}
y^{*}(n)=\alpha^{n}\left(y_{-n}^{*}\right), \quad \alpha \in \operatorname{Aut}(A), \\
y \times z(n)=\sum_{k \in \mathbb{Z}} y_{k} \alpha^{k}\left(z_{n-k}\right) \quad \mathrm{e} \\
\|y\|_{1}=\sum_{n \in \mathbb{Z}}\left|y_{n}\right|
\end{gathered}
$$

para todo $y=\left(y_{n}\right)_{n \in \mathbb{Z}}$ e $z=\left(z_{n}\right)_{n \in \mathbb{Z}}$ em $l_{1}(\mathbb{Z}, A)$ e $n \in \mathbb{Z}$. Temos que $k(\mathbb{Z}, A)$, funções de $l_{1}(\mathbb{Z}, A)$ que tem suporte finito, é um subconjunto fortemente denso de $l_{1}(\mathbb{Z}, A)$.

Sejam $a \in A$ e $n \in \mathbb{Z}$. Denote por $a \delta_{n}$ o elemento de $l_{1}(\mathbb{Z}, A)$ dado por

$$
a \delta_{n}(k)=\left\{\begin{array}{lll}
0 & \text { se } & k \neq n \\
a & \text { se } & k=n
\end{array}\right.
$$

Note que $\left(a \delta_{n}\right)^{*}=\alpha^{-n}\left(a^{*}\right) \delta_{-n}$ e que $\left(a \delta_{n}\right) \times\left(b \delta_{m}\right)=a \alpha^{n}(b) \delta_{n+m}$ para todo $n, m \in \mathbb{Z}$ e todo $a, b \in A$. E não é difícil mostrar que o conjunto $\left\{x \delta_{0}, 1 \delta_{1}: x \in A\right\}$ gera $l_{1}(\mathbb{Z}, A)$ como álgebra de Banach.

\subsection{A estrutura de produto cruzado de $\mathcal{A}^{\diamond}$}

Seja $A=C S(\mathbb{R})$, e consideremos a sequência exata curta

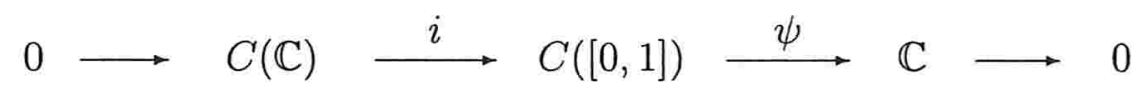

onde $C(\mathbb{C})$ é o cone da álgebra $\mathbb{C}$, isto é $C(\mathbb{C})=\{f:[0,1] \rightarrow \mathbb{C}: f(0)=0\} ; \psi(f)=f(0)$ e $i$ é a inclusão. Aplicando a sequência exata cíclica de seis termos para K-teoria na sequência acima e usando que $K_{0}(C(\mathbb{C}))=K_{1}(C(\mathbb{C}))=0$, veja exemplos 4.1 .5 e 8.3 de [37], obtemos $K_{0}(C([0,1]))=\mathbb{Z}[1]_{0}$ e $K_{1}(C([0,1]))=0$. Como $A$ é isomorfo a $C([0,1])$ temos que $K_{1}(A)=0$ e $K_{0}(A)=\mathbb{Z}[1]_{0}$.

Consideremos $\alpha: \mathbb{Z} \longrightarrow A u t(A)$ onde $n \longmapsto[\alpha(n) a](x)=a(x-n)$. A aplicação $\alpha$ está bem definida e temos que $[\alpha(n+m)(a)](x)=a(x-n-m)=[\alpha(n) \circ \alpha(m)(a)](x)$ para todo $n, m \in \mathbb{Z}$. Logo $\alpha$ é um homomorfismo de grupo. Como $\mathbb{Z}$ é um grupo discreto temos que $\alpha$ é contínua, $\log o \alpha$ é uma ação.

Usando a sequência exata de Pimsner-Voiculescu [34] temos 


$$
\begin{array}{ccc}
K_{0}(A) \stackrel{i d-\alpha_{*}^{-1}}{\longrightarrow} K_{0}(A) & \stackrel{i_{*}}{\longrightarrow} K_{0}(A \underset{\alpha}{\rtimes} \mathbb{Z}) \\
\uparrow_{\delta_{1}^{\prime}} & & \delta_{0}^{\prime} \\
K_{1}(A \underset{\alpha}{\rtimes} \mathbb{Z}) \stackrel{i_{*}}{\longleftarrow} K_{1}(A) \stackrel{i d-\alpha_{*}^{-1}}{\longleftarrow} K_{1}(A)
\end{array}
$$

Como $\alpha(n)(1)(x)=1(x-n)=1$, segue que $\alpha_{*}$ é igual a identidade de $K_{0}(A)$. Usando que $K_{1}(A)=0$ e que $K_{0}(A)=\mathbb{Z}[1]_{0}$ ficamos com a seguinte sequência exata

$$
0 \longrightarrow K_{1}(A \underset{\alpha}{\rtimes} \mathbb{Z}) \stackrel{\delta_{1}^{\prime}}{\longrightarrow} \mathbb{Z}[1]_{0} \stackrel{0}{\longrightarrow} \mathbb{Z}[1]_{0} \stackrel{i_{*}}{\longrightarrow} K_{0}(A \underset{\alpha}{\rtimes} \mathbb{Z}) \longrightarrow 0
$$

Podemos concluir então que

$$
\begin{aligned}
& K_{1}(A \underset{\alpha}{\rtimes} \mathbb{Z}) \cong \mathbb{Z} \quad \mathrm{e} \\
& K_{0}(\underset{\alpha}{\rtimes} \mathbb{Z})=\mathbb{Z}[1]_{0} .
\end{aligned}
$$

Se $B$ é uma $C^{*}$ - álgebra e $\alpha \in A u t(B)$ sempre existe um unitário $u \in B \underset{\alpha}{\rtimes} \mathbb{Z}$ tal que $\alpha(a)=u a u^{*}$ para todo $a \in B$ (veja, por exemplo, [6], pág. 49). Diz-se que um tal $u$ implementa a ação $\alpha$ e que $\alpha$ é o automorfismo induzido por $u$. No nosso caso, temos que $\alpha(n) a=\delta_{1}^{n} a(M)\left(\delta_{1}^{*}\right)^{n}$ para todo $a \in A$ e $n \in \mathbb{Z}$, isto é $\alpha$ é o automorfismo induzido pelo unitário $\delta_{1} \in \underset{\alpha}{x} \underset{\mathbb{Z}}{\mathbb{Z} \text {. }}$

Pela equação apresentada no começo da página 102 de [34] temos que $\delta_{1}^{\prime}\left(\left[\left(1 \otimes 1_{n}-\right.\right.\right.$ $\left.\left.F)+F x\left(u^{*} \otimes 1_{n}\right) F\right]_{1}\right)=[F]_{0}$, onde $F, x \in A \otimes M_{n}, n \in \mathbb{N} ; F$ é uma projeção e $u$ é o unitário que implementa a ação. Consideremos $F=1_{A}=x$ e $n=1$, pela equação acima temos que $\delta_{1}^{\prime}\left(\left[\delta_{-1}\right]_{1}\right)=[1]_{0}$. E como foi visto acima que $\delta_{1}^{\prime}$ é um isomorfismo temos que

$$
K_{1}(A \underset{\alpha}{\rtimes} \mathbb{Z})=\mathbb{Z}\left[\delta_{-1}\right]_{1}
$$

Consideremos a $C^{*}$ - álgebra $\mathcal{A}^{\circ}$ definida na Proposição 1.26. Vamos verificar na Proposição abaixo que $\mathcal{A}^{\circ}$ é isomorfo a $A \underset{\alpha}{\rtimes} \mathbb{Z}$.

Proposição 4.1 Seja $A=C S(\mathbb{R})$, então existe $\varphi: A \underset{\alpha}{\rtimes} \mathbb{Z} \longrightarrow \mathcal{A}^{\circ}$ isomorfismo.

\section{Demonstração:}

Seja $B$ o fecho do conjunto $B_{0}=\left\{\sum_{j \in F} a_{j}(M) T_{j}: a_{j} \in C S(\mathbb{R})\right\}$, onde $F \subset \mathbb{Z}$ finito. 
Daqui até o fim dessa demonstração estaremos considerando $T_{j} u(x)=u(x-j)$.

Consideremos $f: k(\mathbb{Z}, A) \longrightarrow B_{0}$ onde $\left(a_{j}\right)_{j} \longmapsto \sum_{j \in F} a_{j}(M) T_{j}, F \subset \mathbb{Z}$ finito. Vamos verificar que $f$ é um * - homomorfismo contínuo:

$\triangleright$ Produto $f\left(\left(a_{j}\right)_{j}\right) f\left(\left(b_{j}\right)_{j}\right)=\sum_{j} \sum_{k} a_{j}(M) T_{j} b_{k}(M) T_{k}$. Temos que $T_{j} b_{k}(M)=b_{k}(M-j) T_{j}$, então $f\left(\left(a_{j}\right)_{j}\right) f\left(\left(b_{j}\right)_{j}\right)=\sum_{j} \sum_{k} a_{j}(M) b_{k}(M-j) T_{j+k}$. Fazendo mudanças de variáveis e usando a definição de $\alpha$ temos $f\left(\left(a_{j}\right)_{j}\right) f\left(\left(b_{j}\right)_{j}\right)=\sum_{j}\left(\sum_{l} a_{l} \alpha^{l}\left(b_{j-l}\right)\right) T_{j}=f\left(\left(a_{j} * b_{j}\right)_{j}\right)$.

$\triangleright$ Involução

$f\left(\left(a_{j}\right)_{j}\right)^{*}=\sum_{j} T_{-j} a_{j}^{*}(M)=\sum T_{j^{\prime}} a_{-j^{\prime}}^{*}(M)=\sum \alpha^{j^{\prime}}\left(a_{-j^{\prime}}^{*}\right) T_{j^{\prime}}=f\left(\left(a_{j}\right)^{*}\right)$.

$\triangleright$ Continuidade

$\left\|f\left(\left(a_{j}\right)\right)_{j}\right\|=\left\|\sum_{j} a_{j}(M) T_{j}\right\| \leq \sum_{j}\left\|a_{j}(M)\right\|\left\|T_{j}\right\|=\sum_{j}\left\|a_{j}(M)\right\|=\left\|\left(a_{j}\right)_{j}\right\|_{1}$.

Como $f$ é contínua, $k(\mathbb{Z}, A)$ é denso em $l_{1}(\mathbb{Z}, A)$ e $B_{0}$ é denso em $B$ temos que existe * - homomorfismo $\tilde{f}: l_{1}(\mathbb{Z}, A) \longrightarrow B$ extensão contínua de $f$.

$$
\begin{array}{ccc}
k(\mathbb{Z}, A) & \stackrel{f}{\rightarrow} & B_{0} \\
\cap & & \cap \\
l_{1}(\mathbb{Z}, A) & \stackrel{\tilde{f}}{\rightarrow} & B
\end{array}
$$

Por 2.7.4 de [14] temos que existe $\varphi: A \underset{\alpha}{\rtimes} \mathbb{Z} \longrightarrow B *$ - homomorfismo contínuo estendendo $\tilde{f}$.

$$
\begin{array}{cl}
l_{1}(\mathbb{Z}, A) & \stackrel{\tilde{f}}{\rightarrow} \\
\cap & \nearrow \varphi
\end{array}
$$

$A \underset{\alpha}{\rtimes} \mathbb{Z}$

Vamos verificar agora que $\varphi$ é um isomorfismo.

$\triangleright \varphi$ é sobrejetora

Como $\operatorname{Im} \varphi$ é fechada e contém $B_{0}$ que é denso em $B$ temos que $\varphi$ é sobrejetora.

Para provar a injetividade de $\varphi$ vamos utilizar a Proposição 2.9 de [15], que enunciamos abaixo, 
Proposição 4.2 Sejam $C$ e $C^{\prime} C^{*}$-álgebras e sejam $\alpha$ e $\alpha^{\prime}$ ações de $S^{1}$ em $C$ e $C^{\prime}$ respectivamente. Suponha que $\psi: C \longrightarrow C^{\prime}$ é um homomorfismo covariante. Se a restrição de $\psi$ à subálgebra dos pontos fixos $C_{0}$ é injetiva, então $\psi$ é injetiva.

Então vamos definir ações $\gamma$ e $\beta$ de $S^{1}$ em $A \underset{\alpha}{\rtimes} \mathbb{Z}$ e $B$ respectivamente tal que $\beta_{z}(\varphi(x))=\varphi\left(\gamma_{z}(x)\right)$, para todo $z \in S^{1}$ e para todo $x \in \underset{\alpha}{\alpha} \underset{\alpha}{ } \mathbb{Z}$.

Dado $z \in S^{1}$ e $a=\left(a_{j}\right)_{j} \in l_{1}(\mathbb{Z}, A)$ vamos definir $\gamma_{z}(a)=\left(z^{j} a_{j}\right)_{j}$.

Temos que $\gamma$ é uma ação de $S^{1}$ em $l_{1}(\mathbb{Z}, A)$. Seja $i: l_{1}(\mathbb{Z}, A) \longrightarrow A \rtimes \mathbb{Z}$ a inclusão. Então $i \circ \gamma_{z}: l_{1}(\mathbb{Z}, A) \longrightarrow A \rtimes \mathbb{Z}$ é um $*$-homomorfismo contínuo. Por 2.7.4 de [14] temos que $\gamma$ é uma ação de $S^{1}$ em $\underset{\alpha}{A} \underset{\alpha}{ } \mathbb{Z}$.

Dado $z \in S^{1}$ vamos definir o operador $U$ em $\mathcal{L}_{\mathbb{R}}$ por $\left(U_{z} f\right)(t)=z^{t} f(t)$. Temos que $U$ é um operador unitário. Dado $z \in S^{1}$ vamos definir $\beta_{z}(A)=U_{z} A\left(U_{z}\right)^{-1}$ para todo $A \in B$. Temos então que $\beta$ é uma ação de $S^{1}$ em $B$.

Agora vamos verificar que $\varphi$ é um homomorfismo covariante, ou seja, vamos mostrar que $\beta_{z}(\varphi(x))=\varphi\left(\gamma_{z}(x)\right)$, para todo $x \in l_{1}(\mathbb{Z}, A)$. Como $\left\{x \delta_{0}, 1 \delta_{1}: x \in A\right\}$ gera $l_{1}(\mathbb{Z}, A)$, é suficiente verificarmos a equação nesses geradores de $l_{1}(\mathbb{Z}, A)$. Para $x \delta_{0}$ temos que $\gamma_{z}\left(x \delta_{0}\right)=x \delta_{0}$ e $\varphi\left(x \delta_{0}\right)=f\left(x \delta_{0}\right)=x(M), \operatorname{logo}$

$$
\varphi\left(\gamma_{z}\left(x \delta_{0}\right)\right)=x(M)=\beta_{z}\left(\varphi\left(x \delta_{0}\right)\right)
$$

Para $1 \delta_{1}$ temos $\gamma_{z}\left(1 \delta_{1}\right)=z \delta_{1}$ e $\varphi\left(1 \delta_{1}\right)=f\left(1 \delta_{1}\right)=T_{1}$, logo

$$
\varphi\left(\gamma_{z}\left(1 \delta_{1}\right)\right)=z T_{1}=\beta_{z}\left(\varphi\left(1 \delta_{1}\right)\right)
$$

Vamos verificar agora quem é a álgebra dos pontos fixos de $A \rtimes \mathbb{Z}$. Seja $C$ a álgebra dos pontos fixos de $A \rtimes \mathbb{Z}$, temos que $C \cap l_{1}(\mathbb{Z}, A)=A$.

De fato, consideremos a inclusão $i: A \longrightarrow l_{1}(\mathbb{Z}, A)$ onde $x \longmapsto x \delta_{0}$. Se $x \in A$ então pela inclusão temos que $x \in l_{1}(\mathbb{Z}, A)$ e com isso $\gamma_{z}(x)=x$, logo $x \in C \cap l_{1}(\mathbb{Z}, A)$. Seja $x \in C \cap l_{1}(\mathbb{Z}, A)$, temos que $x=\sum a_{j} \delta_{j}$ então $\gamma_{z}(x)=\sum z^{j} a_{j} \delta_{j}$ e como $x \in C$ também temos $\gamma_{z}(x)=x$. Logo $\sum z^{j} a_{j} \delta_{j}=\sum a_{j} \delta_{j}$, para todo $z \in S^{1}$, o que implica que $a_{j} \delta_{j}=0$ ou $z^{j}-1=0$, para todo $z \in S^{1}$. O segundo caso acontece se, e somente se, $j=0$. Portanto $a_{j}=0$ para $j \neq 0, \log 0 x=a_{0} \delta_{0}$, isto é, $x \in A$.

Consideremos agora a aplicação $E: A \underset{\alpha}{\rtimes} \mathbb{Z} \longrightarrow A \underset{\alpha}{x} \mathbb{Z}$ definida por

$$
E(b)=\int_{0}^{2 \pi} \gamma_{z}(b) d z, b \in A \underset{\alpha}{\rtimes} \mathbb{Z} \text { e } d z=\frac{d \theta}{2 \pi} .
$$


Se $b$ é tal que $\gamma_{z}(b)=b$ para todo $z \in S^{1}$ então $E(b)=\int_{0}^{2 \pi} b d z=b, \log 0 C \subset \operatorname{Im} E$. Seja $b=\left(b_{j}\right)_{j} \in l_{1}(\mathbb{Z}, A)$ então

$$
E(b)=\int_{0}^{2 \pi} \gamma_{z}(b) d z=\int_{0}^{2 \pi}\left(z^{j} b_{j}\right)_{j} d z=\left(b_{j} \int_{0}^{2 \pi} z^{j} d z\right)_{j}=b_{0} \delta_{0} \in A .
$$

Então para todo $a \in l_{1}(\mathbb{Z}, A)$ temos que $E(a) \in A$. Como $l_{1}(\mathbb{Z}, A)$ é uma subálgebra densa em $A \rtimes \mathbb{Z}$, temos a imagem de $E$ contida em $A$. Portanto temos $C \subset \operatorname{Im} E \subset A=$ $C \cap l_{1}(\mathbb{Z}, A),{ }^{\alpha} \log 0=A$.

Resta-nos provar que $\varphi$ é injetora na álgebra dos pontos fixos. Consideremos a inclusão $i: A \longrightarrow A \rtimes \mathbb{Z}$ onde $a \longmapsto a \delta_{0}$. Temos que $\varphi\left(a \delta_{0}\right)=f\left(a \delta_{0}\right)=a(M)$ e $\varphi\left(a \delta_{0}\right)=\varphi\left(b \delta_{0}\right) \Leftrightarrow a(M)^{\alpha}=b(M) \Leftrightarrow a=b$.

Portanto $\varphi$ é injetora na álgebra dos pontos fixos, logo pela Proposição 4.2 temos que $\varphi$ é injetora. Logo $\varphi: A \underset{\alpha}{\rtimes} \mathbb{Z} \longrightarrow B$ é um isomorfismo.

Como $\mathcal{A}^{\circ}=F B F^{-1}$ temos que $A \underset{\alpha}{\rtimes} \mathbb{Z}$ é isomorfo a $\mathcal{A}^{\circ}$.

Na página 68 vimos que $K_{0}(A \underset{\alpha}{\rtimes} \mathbb{Z})=\mathbb{Z}[1]_{0}$ e que $K_{1}(A \underset{\alpha}{\rtimes} \mathbb{Z})=\mathbb{Z}\left[\delta_{-1}\right]_{1}, \operatorname{logo}$

$$
\begin{gathered}
K_{0}\left(\mathcal{A}^{\circ}\right)=\mathbb{Z}[I d]_{0} \quad \mathrm{e} \\
K_{1}\left(\mathcal{A}^{\circ}\right)=\mathbb{Z}\left[e^{i M}\right]_{1} .
\end{gathered}
$$

\subsection{A K-teoria de $\mathcal{A} / \mathcal{K}_{\mathbb{R}}$ e $\mathcal{A}$}

Temos que o conjunto

$$
\mathcal{A}_{0}=\left\{\sum_{j=-N}^{N} a_{j}(M) b_{j}(D) e^{i j M}+K ; N \in \mathbb{N}, a_{j} \in C S(\mathbb{R}), b_{j} \in C S(\mathbb{R}), K \in \mathcal{K}_{\mathbb{R}}\right\}
$$

é denso em $\mathcal{A}$. Dado $A=\sum_{j=-N}^{N} a_{j}(M) b_{j}(D) e^{i j M} \in \mathcal{A}_{0}$, sejam $A^{ \pm}=\sum_{j=-N}^{N} a_{j}( \pm \infty) b_{j}(D) e^{i j M}$. Temos que $A^{+}$e $A^{-} \in \mathcal{A}^{\circ}$ logo pela demonstração da Proposição 1.26 e pela definição de $\gamma$ temos que $\gamma_{A}=\left(W F^{-1} A^{+}\left(W F^{-1}\right)^{-1}, W F^{-1} A^{-}\left(W F^{-1}\right)^{-1}\right)$.

Como isso vale para todo $A$ em uma subálgebra densa de $\mathcal{A}$, segue que a aplicação

$$
\mathcal{A}_{0} \ni A \longmapsto\left(A^{+}, A^{-}\right) \in \mathcal{A}^{\circ} \oplus \mathcal{A}^{\circ}
$$


se estende a um $*$-homomorfismo

$$
\psi: \mathcal{A} \longrightarrow \mathcal{A}^{\circ} \oplus \mathcal{A}^{\circ}
$$

tal que, para todo $A \in \mathcal{A}$, e com $U=\left(\begin{array}{cc}W F^{-1} & 0 \\ 0 & W F^{-1}\end{array}\right)$ temos $U \psi(A) U^{-1}=\gamma(A)$. Segue então que $\operatorname{Ker} \psi=\operatorname{Ker} \gamma=J_{0}$ (veja Proposição 1.27).

Proposição $4.3 K_{0}\left(\mathcal{A} / \mathcal{K}_{\mathbb{R}}\right)$ é isomorfo a $\mathbb{Z}$ e $K_{1}\left(\mathcal{A} / \mathcal{K}_{\mathbb{R}}\right)$ é isomorfo a $\mathbb{Z} \oplus \mathbb{Z} \oplus \mathbb{Z}$.

\section{Demonstração:}

Consideremos a seguinte sequência exata curta:

$$
0 \longrightarrow J_{0} \stackrel{\iota}{\longrightarrow} \mathcal{A} \stackrel{\psi}{\longrightarrow} \mathcal{A}^{\circ} \oplus \mathcal{A}^{\circ} \longrightarrow 0
$$

onde $\psi(A)=\left(A^{+}, A^{-}\right) \operatorname{com} A$ e $A^{ \pm}$como acima.

Podemos tomar então a seguinte sequência exata curta

$$
0 \longrightarrow J_{0} / \mathcal{K}_{\mathbb{R}} \stackrel{\iota}{\longrightarrow} \mathcal{A} / \mathcal{K}_{\mathbb{R}} \stackrel{\psi}{\longrightarrow} \mathcal{A}^{\circ} \oplus \mathcal{A}^{\circ} \longrightarrow 0
$$

Como $J_{0} / \mathcal{K}_{\mathbb{R}}$ é isomorfo a $C_{0}(\mathbb{R} \times\{-\infty,+\infty\})$ (Lema 1.15) e $C_{0}(\mathbb{R} \times\{-\infty,+\infty\})$ é isomorfo a $C_{0}(\mathbb{R}) \oplus C_{0}(\mathbb{R})$ temos que $K_{1}\left(J_{0} / \mathcal{K}_{\mathbb{R}}\right) \cong \mathbb{Z} \oplus \mathbb{Z}$ e $K_{0}\left(J_{0} / \mathcal{K}_{\mathbb{R}}\right)=0$.

Usando o isomorfismo definido no Lema 1.15 temos que

$$
K_{1}\left(J_{0} / \mathcal{K}_{\mathbb{R}}\right)=\mathbb{Z}\left[[U]_{\mathcal{K}_{\mathbb{R}}}\right]_{1} \oplus \mathbb{Z}\left[[V]_{\mathcal{K}_{\mathbb{R}}}\right]_{1}
$$

onde $U=e^{2 \pi i b(M)} b(D)+c(D)$ e $V=e^{2 \pi i b(M)} c(D)+b(D)$ com $b$ e $c$ as funções definidas na página 54 .

Observemos que $U$ e $V$ pertencem a $J_{0} \oplus \mathbb{C} I d \subset \mathcal{L}\left(L^{2}(\mathbb{R})\right)$, pois (como $b+c \equiv 1$ ) $I d-U=\left(e^{2 \pi i b(M)}-I d\right) b(D)=\tilde{b}(M) b(D)$ com $\tilde{b} \in C_{0}(\mathbb{R})$, e analogamente para $V$. Como, por definição, $K_{1}\left(J_{0} / \mathcal{K}_{\mathbb{R}}\right)=K_{1}\left(\widetilde{J_{0} / \mathcal{K}_{\mathbb{R}}}\right)$, segue que, de fato, $\left[[U]_{\mathcal{K}_{\mathbb{R}}}\right]_{1}$ e $\left[[V]_{\mathcal{K}_{\mathbb{R}}}\right]_{1}$ são elementos de $K_{1}\left(J_{0} / \mathcal{K}_{\mathbb{R}}\right)$.

Aplicando a sequência exata cíclica de seis termos para K-teoria na sequência acima obtemos 


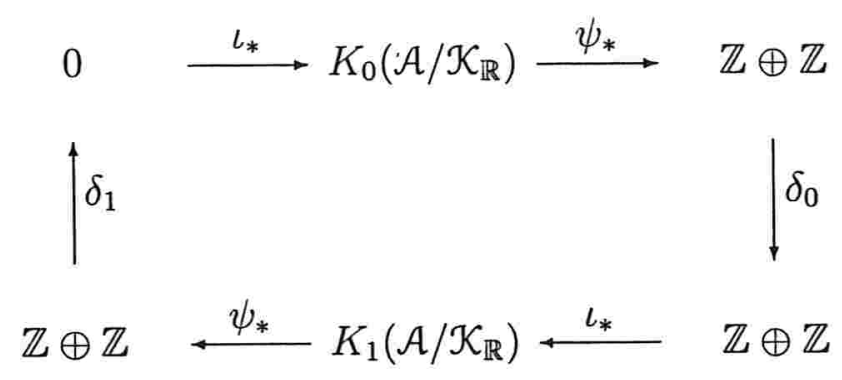

Logo $\operatorname{Im} \delta_{1}=0$ e $\operatorname{Ker} \delta_{1}=\mathbb{Z} \oplus \mathbb{Z}$.

Seja $\mathcal{C}$ a $C^{*}$ - álgebra do Capítulo 2 , neste capítulo vimos que $\mathcal{C} / \mathcal{K}_{\mathbb{R}} \cong C\left(M_{c}\right)$ onde $M_{c}=\{(x, \xi) \in[-\infty,+\infty] \times[-\infty,+\infty]:|x|+|\xi|=\infty\}$ e que $K_{1}\left(\mathcal{C} / \mathcal{K}_{\mathbb{R}}\right)=$ $\mathbb{Z}\left[\left[e^{2 \pi i c(M)} b(D)+c(D)\right]_{\mathcal{K}_{\mathbb{R}}}\right]_{1}$.

Consideremos as inclusões

$$
J_{0} / \mathcal{K}_{\mathbb{R}} \stackrel{i}{\longrightarrow} \mathrm{C} / \mathcal{K}_{\mathbb{R}} \stackrel{j}{\longrightarrow} \mathcal{A} / \mathcal{K}_{\mathbb{R}}
$$

Temos que $\iota=j \circ i$. Pela definição de $\psi$ temos que $\psi_{*}\left([I d]_{0}\right)=\left([I d]_{0},[I d]_{0}\right)$. Logo com respeito ao isomorfismo $K_{0}\left(\mathcal{A}^{\circ} \oplus \mathcal{A}^{\circ}\right) \cong \mathbb{Z} \oplus \mathbb{Z}$ podemos escrever que $(1,1) \in \operatorname{Im} \psi_{*}=\operatorname{Ker} \delta_{0}$ e $\delta_{0}(1,1)=0$.

Seja $W=e^{2 \pi i c(M)} c(D)+b(D)$. Calculando o número de rotação de $\sigma_{U}$ e $\sigma_{W}$ temos que $w\left(\sigma_{U}\right)=w\left(\sigma_{W}\right)=-1$. Logo pelo o que vimos na página 47 sobre o número de rotação temos que $i_{*}\left(\left[[U]_{\mathcal{K}_{\mathbb{R}}}\right]_{1}\right)=i_{*}\left(\left[[W]_{\mathcal{K}_{\mathbb{R}}}\right]_{1}\right)=\left[\left[e^{2 \pi i c(D)} b(M)+c(M)\right]_{\mathcal{K}_{\mathbb{R}}}\right]_{1}$. Portanto com respeito ao isomorfismo $K_{1}\left(J_{0} / \mathcal{K}_{\mathbb{R}}\right)=\mathbb{Z}\left[[U]_{\mathcal{K}_{\mathbb{R}}}\right]_{1} \oplus \mathbb{Z}\left[[V]_{\mathcal{K}_{\mathbb{R}}}\right]_{1}$ temos que $\iota_{*}((1,0)-$ $(0,-1))=j_{*} \circ i_{*}((1,0)-(0,-1))=0$. Logo $(1,1) \in \operatorname{Ker} \iota_{*}=\operatorname{Im} \delta_{0}$.

Suponhamos que $\delta_{0}(1,0)=(m, n)$, como $(x, y)=(x-y)(1,0)+y(1,1)$ temos que $\delta_{0}(x, y)=(x-y)(m, n)$, para todo $(x, y) \in \mathbb{Z} \oplus \mathbb{Z}$.

Como $(1,1) \in \operatorname{Im} \delta_{0}$ temos que existe $(x, y) \in \mathbb{Z} \oplus \mathbb{Z}$ tal que $(x-y)(m, n)=(1,1)$, isto é, $(x-y) m=1$ e $(x-y) n=1$. Logo $m=n \neq 0$ e com isto $\operatorname{Im} \delta_{0}=\mathbb{Z}(m, n)$ e Ker $\delta_{0}=$ $\mathbb{Z}(1,1)$. Sabemos que $(1,1) \in \operatorname{Ker} \iota_{*}=\operatorname{Im} \delta_{0} \cong \mathbb{Z}$. Logo, $\operatorname{Im} \delta_{0}=\operatorname{Ker} \iota_{*}=(1,1) \mathbb{Z}$.

Podemos então concluir pelo Teorema do homomorfismo que $\operatorname{Im} \iota_{*} \cong \mathbb{Z}(1,0)$, onde $(1,0) \in K_{1}\left(J_{0} / \mathcal{K}_{\mathbb{R}}\right)=\mathbb{Z}\left[[U]_{\mathcal{K}_{\mathbb{R}}}\right]_{1} \oplus \mathbb{Z}\left[[V]_{\mathcal{K}_{\mathbb{R}}}\right]_{1}$.

Pela exatidão da sequência exata ciclíca de seis termos temos que $\psi_{*}: K_{0}\left(\mathcal{A} / \mathcal{K}_{\mathbb{R}}\right) \longrightarrow$ $\operatorname{Im} \psi_{*}=\mathbb{Z}(1,1)$ é um isomorfismo. Logo $K_{0}\left(\mathcal{A} / \mathcal{K}_{\mathbb{R}}\right)=\mathbb{Z}\left[[I d]_{\mathcal{K}_{\mathbb{R}}}\right]_{0}$. 
Como $\delta_{1} \equiv 0$ temos que $\operatorname{Im} \psi_{*} \cong \mathbb{Z} \oplus \mathbb{Z} \cong K_{1}\left(\mathcal{A}^{\circ} \oplus \mathcal{A}^{\circ}\right)$. Então podemos construir a seguinte sequência exata curta

$$
0 \longrightarrow \mathbb{Z}(1,0) \stackrel{i}{\longrightarrow} K_{1}\left(\mathcal{A} / \mathcal{K}_{\mathbb{R}}\right) \stackrel{\psi_{*}}{\longrightarrow} \mathbb{Z} \oplus \mathbb{Z} \longrightarrow 0
$$

Logo $K_{1}\left(\mathcal{A} / K_{\mathbb{R}}\right)=\mathbb{Z}\left[[A]_{\mathcal{K}_{\mathbb{R}}}\right]_{1} \oplus \mathbb{Z}\left[[B]_{\mathcal{K}_{\mathbb{R}}}\right]_{1} \oplus \mathbb{Z}\left[[U]_{\mathcal{K}_{\mathbb{R}}}\right]_{1}$ onde $A$ e $B$ são elementos de $\mathcal{A}$ tal que $\psi(A)=\left(e^{i M}, 1\right)$ e $\psi(B)=\left(1, e^{i M}\right)$. Podemos tomar, por exemplo, $A=L(M) \mathrm{e}$ $B=\tilde{L}(M), L$ e $\tilde{L}$ definidas na página 54 .

Agora já é possivel calcular a K-teoria da $C^{*}$ - álgebra $\mathcal{A}$.

Teorema 4.4 Seja $\pi: \mathcal{A} \longrightarrow \mathcal{A} / \mathcal{K}_{\mathbb{R}}$ a projeção canônica. Então $\pi_{*}: K_{i}(\mathcal{A}) \longrightarrow \operatorname{Im} \pi_{*} e ́$ um isomorfismo.

\section{Demonstração:}

Consideremos a sequência exata curta

$$
0 \longrightarrow \mathcal{K}_{\mathbb{R}} \stackrel{i}{\longrightarrow} \mathcal{A} \stackrel{\pi}{\longrightarrow} \mathcal{A} / \mathcal{K}_{\mathbb{R}} \longrightarrow 0
$$

e sua sequência exata ciclíca de seis termos para K-teoria

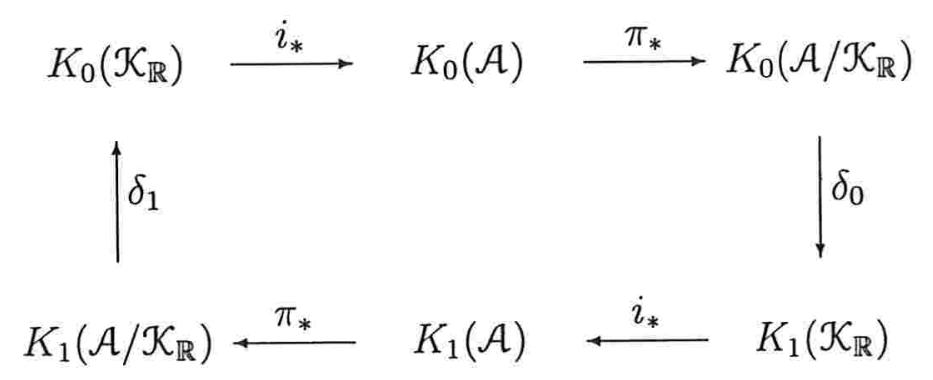

Substituindo as K-teorias conhecidas temos

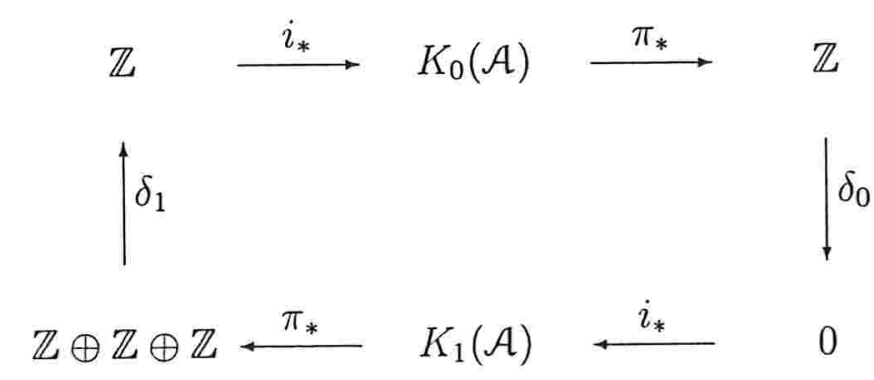

Sabemos pela Proposição 9.4.4 de [37] que o índice para esta sequência é o índice de Fredholm. 
Vamos calcular $\delta_{1}$ nos três geradores de $K_{1}\left(\mathcal{A} / \mathcal{K}_{\mathbb{R}}\right)$. Temos que $\delta_{1}\left(\left[[L(M)]_{\mathcal{K}_{\mathbb{R}}}\right]_{1}\right)=$ $\delta_{1}\left(\left[[\tilde{L}(M)]_{\mathcal{K}_{\mathbb{R}}}\right]_{1}\right)=0$ já que eles são operadores de multiplicação.

É fácil ver que $e^{2 \pi i b(M)} b(D)+c(D) \in \mathcal{C} \operatorname{logo}\left[e^{2 \pi i b(M)} b(D)+c(D)\right]_{\mathcal{K}_{\mathbb{R}}} \in \mathcal{C} / \mathcal{K}_{\mathbb{R}}$ e como foi visto no Capítulo 2, Teorema 2.13, $\delta_{1}\left(\left[[T]_{\mathcal{K}_{\mathbb{R}}}\right]_{1}\right)=\operatorname{ind}(T)=w\left(\sigma_{T}\right)$ nesta álgebra. Logo $\delta_{1}\left(\left[\left[e^{2 \pi i b(M)} b(D)+c(D)\right]_{\mathcal{K}_{\mathbb{R}}}\right]_{1}\right)=w\left(\sigma_{e^{2 \pi i b(M)} b(D)+c(D)}\right)=-1$. Portanto $\delta_{1}$ é sobrejetora e $\delta_{1}(x, y, z)=-z$.

Usando a sequência exata cíclica de seis termos temos

$$
\begin{gathered}
K_{0}(\mathcal{A})=\mathbb{Z}[I d]_{0} \quad \mathrm{e} \\
K_{1}(\mathcal{A})=\mathbb{Z}[L(M)]_{1} \oplus \mathbb{Z}[\tilde{L}(M)]_{1} .
\end{gathered}
$$




\section{Referências Bibliográficas}

[1] M. Atiyah, I. Singer, The index of elliptic operators on compact manifolds, Bull. Amer. Math. Soc. 69, 422-433, 1963.

[2] M. Atiyah, I. Singer, The index of elliptic operators I, Ann. of Math 87, 2, 484-530, 1968.

[3] M. Atiyah, I. Singer, The index of elliptic operators III, Ann. of Math 87, 2, 546-604, 1968.

[4] B. Blackadar, K-theory for operator algebras, Cambridge University Press, Cambridge, 1998.

[5] D. Bleecker, B. Booss-Bavnbek, Topology and analysis: The Atiyah-Singer index formula and Gauge-theoretic physics, Springer-Verlag, New York, 1985.

[6] C. Cerri, Certas deformações não-comutativas do toro e sua k-teoria, Tese (doutorado), IME-USP, 1993.

[7] A. Connes, Gravity coupled with matter and the foundation of non-commutative geometry, Comm. Math. Phys. 182, 155-176, 1996.

[8] A. Connes e H. Moscovici, Cyclic cohomology, the Novikov conjecture and hyperbolic groups, Topology 29, 345-388, 1990.

[9] H. O. Cordes, Spectral theory of linear differential operators and comparison algebras, London Mathematical Society Lecture Note Series 76, Cambridge University Press, Cambridge, 1987. 
[10] H. O. Cordes, Elliptic pseudodifferential operators - an abstract theory, Lecture Notes in Mathematics 756, Springer, Berlin, 1979.

[11] H. O. Cordes e M. Breuer, On Banach algebras with $\sigma$ symbol II, J. Math. Mech. 14, 299-313, 1965.

[12] H. O. Cordes e E. A Herman, Gelfand theory of pseudo differential operators, Amer. J. Math. 90, 681-717, 1968.

[13] H. O. Cordes e S. T. Melo, An algebra of singular integral operators with kernels of bounded oscillation and application to periodic differential operators, Journal of Differential Equations 75-2, 216-238, 1987.

[14] J. Diximier, Les $C^{*}$-algèbres et leurs représentations, Gauthier-Villars, Paris, 1969.

[15] R. Exel, Circle actions on $C^{*}$-algebras, partial automorphisms and a generalized Pimsner-Voiculescu exact sequence, J. Funct. Anal. 122, no. 2, 361-401, 1994.

[16] P. Gilkey, Invariance theory, the heat equation, and the Atiyah-Singer index theorem, Mathematics Lecture Series 11, Publish or Perish, 1984.

[17] H. Helson, Harmonic analysis, Addison-Wesley, Mass., 1983.

[18] L. Hormander, The analysis of linear partial differential operators III, SpringerVerlag, New York, 1990.

[19] J. Hounie, Teoria elementar das distribuições, Colóquio Brasileiro de Matemática, Impa, Rio de Janeiro, 1979.

[20] R. Lauter, Holomorphic functional calculus in several variables and $\Psi^{*}$ - álgebras of totally characteristic operators on manifolds with boundary, Thesis, Mainz, 1996.

[21] R. Lauter e S. Moroianu, Homology of pseudodifferential operators on manifolds with fibered cusps, Trans. Amer. Math. Soc. 355, no. 8, 3009-3046, 2003.

[22] R. Lauter e S. Moroianu, The index of cups operators on manifolds with corners, Ann. Global Anal. Geom. 21, 31-39, 2002.

[23] S. T. Melo, A compararison algebra on a cylinder with semi-periodic multiplications, Pacific J. Math. 146-2, 281-304, 1990. 
[24] S. T. Melo, R. Nest e E. Schrohe, $C^{*}$ - structure and K-theory of Boutet de Monvel's algebra, J. Reine Angew. Math. 561, 145-175, 2003.

[25] R. Melrose e V. Nistor, $K$-theory of $C^{*}$-algebras of b-pseudodifferential operators, Geom. Funct. Anal. 8, no. 1, 88-122, 1998.

[26] B. Monthubert, Groupoïdes et calcul pseudo-différentiel sur les variétés à coins, Thesis, Paris 7, 1998.

[27] B. Monthubert, Pseudodifferential calculus on manifolds with corners and groupoids, Proc. Amer. Math. Soc. 127, no. 10, 2871-2881, 1999.

[28] G. J. Murphy, $C^{*}$-algebras and operator theory, Academic Press, Boston, 1990.

[29] R. Nest e B. Tsygan, Algebraic index theorem, Comm. Math Phys. 172, 2, 223-262, 1995.

[30] R. Nest e B. Tsygan, Algebraic index theorem for familes, Adv. Math. 113, 2, 151-205, 1995.

[31] R. Nest e B. Tsygan, Formal versus analytic index theorem, Internat. Math. Res. Notices 11, 557-564, 1996.

[32] V. Nistor, Higher index theorems and the boundary map in cyclic cohomology, Documenta Math. 2, 263-295, 1997.

[33] G. Pedersen, $C^{*}$ algebras and their automorphism groups, Academic Press, London, 1979.

[34] M. Pimsner e D. Voiculescu, Exact sequences for K-groups and Ext-groups of certain cross-product $C^{*}$-algebras., J. Operator Theory 4, no. 1, 93-118, 1980.

[35] S. C. Power, Commutator ideals and pseudodifferential $C^{*}$-algebras, Quart. J. Math. Oxford Ser. (2) 31, no. 124, 467-489, 1980.

[36] M. Reed e B. Simon, Methods of modern mathematical physics, Academic Press, New York, 1972.

[37] M. Rørdam, F. Larsen, N. Laustsen, An introduction to K-theory for $C^{*}$-algebras, Cambridge University Press, Cambridge, 2000. 
[38] W. Rudin, Real and complex analysis, Tata McGraw-Hill, New Delhi, 1981. 